Neuronal Activity and Ion Homeostasis in the Hypoxic Brain

\author{
Bas-Jan Zandt
}




\section{Samenstelling promotiecommissie:}

Prof. dr. Gerard van der Steenhoven (voorzitter)

Prof. dr. ir. Michel J.A.M. van Putten (promotor)

Dr. Bennie ten Haken (assistent promotor)

Prof. dr. Stephan van Gils

Prof. dr. Richard van Wezel

Dr. Jeannette Hofmeijer

Prof. dr. Wytse Wadman

Prof. dr. Rudolf Graf

Prof. dr. Michael Müller
Universiteit Twente

Universiteit Twente

Universiteit Twente

Universiteit Twente

Universiteit Twente

Universiteit Twente

Universiteit van Amsterdam

Max-Planck-Institut für neurologische Forschung, Köln

Universitätsmedizin Göttingen

The work in this thesis was carried out at the Neuroimaging group, in close collaboration with the Clinical Neurophysiology group, both of the Faculty of Science and Technology, and the MIRA Institute for Biomedical Engineering and Technical Medicine, at the University of Twente. This work was financially supported by het Ministerie van Economische Zaken, Provincie Overijssel and Provincie Gelderland through the ViPBrainNetworks project.

Nederlandse titel:

Neuronale activiteit en ionen homeostase in het hypoxische brein

\section{Publisher:}

Bas-Jan Zandt, Neuroimaging group, University of Twente, P.O.Box 217, 7500AE Enschede, The Netherlands

http://www.utwente.nl/tnw/nim/

b.zandt@alumnus.utwente.nl

Cover design: Bas-Jan Zandt

Printed by: Gildeprint Drukkerijen - Enschede

(c) Bas-Jan Zandt, Enschede, The Netherlands, 2014.

No part of this work may be reproduced by print, photocopy or any other means without the permission in writing from the publisher.

ISBN: 978-90-365-3599-1

DOI: http://dx.doi.org/10.3990/1.9789036535991 


\title{
NEURONAL ACTIVITY AND ION HOMEOSTASIS IN THE HYPOXIC BRAIN
}

\author{
PROEFSCHRIFT
}

ter verkrijging van

de graad van doctor aan de Universiteit Twente, op gezag van de rector magnificus,

Prof. dr. H. Brinksma, volgens besluit van het College voor Promoties in het openbaar te verdedigen op vrijdag 21 februari 2014 om 16.45 uur

$$
\text { door }
$$

Bas-Jan Zandt

geboren op 24 januari 1985

te Kampen 
Dit proefschrift is goedgekeurd door de promotor:

Prof. Dr. Ir. Michel J.A.M. van Putten

en de assistent-promotor:

Dr. Bennie ten Haken 
To those who taught me and made me who I am 



\section{Contents}

1 Introduction and scope 1

$\begin{array}{lll}2 & \text { Pathophysiology of ischemic stroke } & 7\end{array}$

3 Neural Dynamics during Anoxia and the "Wave of Death" 21

4 Diffusing substances during spreading depolarization 35

5 Single neuron dynamics during experimentally induced anoxic depolarization $\quad 59$

6 A neural mass model based on single cell dynamics to model pathologies 75

$\begin{array}{lll}7 & \text { General Discussion and Outlook } & 107\end{array}$

$\begin{array}{ll}\text { Summary } & 117\end{array}$

$\begin{array}{ll}\text { Samenvatting } & 119\end{array}$

$\begin{array}{ll}\text { Dankwoord (Acknowledgements) } & 121\end{array}$

$\begin{array}{lr}\text { Publications and Contributions } & 125\end{array}$

$\begin{array}{ll}\text { About the author } & 127\end{array}$ 


\section{1}

\section{Introduction and scope}

The brain uses large amounts of oxygen and glucose, which are continuously supplied by the blood stream. It consumes up to twenty percent of the total energy production of the body. Besides consuming much energy, the brain is also very vulnerable for temporary lack of blood flow (ischemia) and the resulting lack of glucose and oxygen (anoxia/hypoxia). Already after several minutes, a lack of energy supply induces irreversible damage. The most common causes of ischemia in the brain are cardiac arrest, resulting in global ischemia, and stroke, resulting in focal ischemia.

Cerebral ischemic damage resulting from stroke or cardiac arrest is the leading cause of death and disability in the world. It has major impact on the quality of life of survivors and their caretakers. It also has significant economic impact due to lost productivity and health care costs. Despite improved preventive treatments (e.g. blood pressure regulation), the number of strokes steadily increases due to ageing of the population. The current yearly number of deaths caused by cerebrovascular diseases worldwide is estimated at 17 million [1,2].

Focal ischemia results in an infarct, consisting of a core of dead tissue, with a surrounding "penumbra" of tissue that is functionally impaired, but can in principle be salvaged. In the first hours to days, the infarct core progresses into the penumbra. Treatments that successfully prevent this delayed cell death are still not available. In the last decades, more than 1000 neuroprotective agents have been proposed and several were tested successfully in animals. However, none of the more than 100 agents that made it to clinical trials were successful in human patients [3, 4]. Possible 
reasons for the failure to translate these treatments from animal to patient studies are differences in lesion size, composition of brain tissue or timing of drug delivery [4-6]. Knowledge of the dynamics of the (patho)physiological processes occurring during and after ischemia is argued to be necessary to successfully design therapies and new medication [7].

Many of the individual processes playing a role have already been identified. These include cerebral energy consumption and metabolism, neuronal membrane voltage dynamics and action potential generation, synaptic functioning, changes in extra- and intracellular concentrations (ions, molecular messengers, $\mathrm{pH}$ ), glial uptake and blood flow regulation [8]. However, the dynamics of the interplay of these processes is largely unknown. As a consequence, the effect of a therapeutic intervention is hard to predict.

One goal of this work is to describe secondary cell death in the penumbra, and investigate how therapies can reduce this. An approach to better identify the key processes and parameters resulting in secondary damage, and the influence of medication or hypothermia on these, is mathematical modeling. This work focuses on modeling the dynamics of excitotoxicity and neuronal depolarization, i.e. the overstimulation and subsequent depolarization of neurons by extracellular potassium and glutamate, that are released following ischemia.

The intended mechanism of several proposed neuroprotective agents is to reduce excitotoxity and neuronal depolarization $[3,4]$. These agents, typically channel blockers or antagonists, prevent release of excitatory substances, block excitatory receptors, and/or reduce excitability of the neurons [3].

To predict how a neuroprotective agent affects the dynamics of infarct progression, a model is needed for which, first, it is clear how the action of the agent can be included, and second, the dynamics can be mathematically analyzed. Existing mathematical models of ischemic stroke have either property, but not both. On the one hand there are detailed, biophysical models in which all variables denote a concrete quantity, such as the concentration of a substance or the flux of ions between compartments. The advantage of such models that explicitly describe biophysical processes, is the simplicity with which e.g. channel blockers can be introduced, allowing for "in silico" experiments. On the other hand there are more phenomenological models, which describe the processes occurring in the infarcted tissue more abstract. These enable analysis of the general dynamics.

Dronne et al. [6], for example, have modeled ion movements between neurons, glia and extracellular space and the resulting cell swelling following occlusion of a blood vessel. Their model includes 30 ion channels, pumps, exchangers and receptors. They show that an a-specific sodium channel blocker drastically reduces cell swelling in ischemic cerebral tissue of rat, but using parameters for human grey matter, they find that sodium inflow persists through NMDA channels and cell swelling 
is only slightly reduced. The complexity of this detailed model, however, makes it difficult to analyze the underlying dynamics. Furthermore, including all relevant interactions at a similar detailed level for e.g. diffusion, synaptic activity, metabolism and cell damage, would result in a very complicated and impractical model.

The model of Vatov et al. $[9,10]$ is an example of a more phenomenological model. It represents extracellular potassium, metabolic stores and cell damage with variables with values normalized to one. The time courses of these variables are calculated from biophysically motivated, but simple, phenomenological expressions. For example, potassium release is described as a polynomial function of the potassium concentration $\left(\left[\mathrm{K}^{+}\right]_{e}\right)$. This captures the qualitative behavior of $\left[\mathrm{K}^{+}\right]_{e}$, that is restored to a resting value when disturbed, but increases fast when a threshold value is crossed. Their model shows how spreading depolarization waves originate from tissue close to the ischemic core, depleting the metabolic stores in the tissue in the penumbra, thereby increasing the infarct size. This more abstract model allows for mathematical analysis of the underlying dynamics. In such a model, however, the link with the physiology is lost and it is not clear how to model the effects of, for example, a channel blocker.

To be able to analyze the dynamics, as well as have a link with the physiological parameters, models on different levels of abstraction can be connected [11]. This work aims to describe an ischemic infarct, using models with physiological parameters whose dynamics can subsequently be analyzed by simplifying the models. In specific, the processes related to excitotoxicity are investigated: the dynamics of ionic homeostasis, the neuronal membrane voltage and energy consumption.

A second motivation for modeling the neuronal activity is to improve diagnostics and prognostication of patients with global ischemic damage. This can elucidate the (patho)physiological processes underlying changes in electroencephalogram (EEG) dynamics. Patients in the intensive care unit treated with therapeutic hypothermia after cardiac arrest, are increasingly monitored with EEG. Typically, several features of the signal are evaluated, such as the signal amplitude, mean frequency or presence of burst-suppression patterns. This yields valuable information for prognostication [12]. The interpretation of the EEG, however, is mainly phenomenological in current practice and the underlying generating mechanisms of the various EEG patterns are largely unknown.

The dynamics of the macroscopic brain rhythms observed in the EEG are reproduced by so-called neural mass models (NMM) or mean field models [13]. NMM successfully describe rhythms and reactions to stimuli in the healthy brain [14]. Furthermore, existing work has already included alterations of the synaptic responses in NMMs. Hindriks and van Putten [15] show how the prolonged synaptic response induced by propofol changes the power spectrum of the EEG. Cloostermans et al. show that a progressive number of failing inhibitory synapses results in the gener- 
alized periodic discharges observed in postanoxic patients [16]. Also, methods are available to estimate patient specific parameters. Aarabi and He [17] estimate model parameters, excitability of the neuronal populations and synaptic strengths [18, 19], from EEGs of patients with epilepsy.

After ischemia, not only the synaptic, buy also the single cell dynamics are altered due to pathophysiological and pharmacological changes. The relation between the population dynamics and the single cell parameters, e.g. membrane conductances and ionic reversal potentials, is unclear.

In this thesis, the single cell dynamics will be included explicitly in a NMM, allowing the observed EEG dynamics to be related to the processes occurring in the post-anoxic brain. The influence of ion concentrations and ATP availability on the single neuron dynamics is investigated first. Subsequently, the relation with the dynamics with the EEG is investigated using neural mass modeling.

\subsection{Scope and set up of the thesis}

The work in this thesis focuses on the subacute phase, the minutes to hours after the ischemic/hypoxic insult. Not discussed will be opportunities for stroke prevention, e.g. by healthy lifestyle or blood pressure regulators, and therapies in the weeks to months after stroke, e.g. focusing on rejuvenation of damaged tissue or recovery of neurological function.

The dynamics of two processes that play an important role in hypoxia and ischemia in this phase will be investigated in specific: dynamics of the ion concentrations in the intra- and extracellular space and the dynamics of the electrical activity of the neurons in the brain. This thesis will describe the interaction between the two processes and to some extent how these dynamics affect cerebral metabolism and cellular viability.

In Chapter 2 an overview is given of pathophysiology of ischemia: metabolism, ion homeostasis and the interaction with neuronal activity.

In Chapter 3, the direct effects of complete cessation of ion pump activity are modeled. With the model, a peculiar phenomenon is reproduced, the so-called wave of death, that is observed in rats after decapitation. The chapter discusses how this can be caused by cerebral anoxic depolarization, in which the neurons in the brain depolarize en masse after having been silent for approximately a minute.

In Chapter 4, initiation and propagation of spreading depolarization is investigated, a slow wave of depolarizing neurons. Simplified expressions will be derived that relate the wave form, propagation velocity, and triggering threshold to four physiological parameters: the diffusion constant, the release rate and removal rate of potassium and/or glutamate and the concentration threshold above which neurons are excited. 
Chapter 5 validates the mathematical models used for single cell dynamics during depolarization with in vitro experiments. In these experiments, the sodium-potassium pumps of neurons in slices from rat brain were blocked. The various types of membrane voltage dynamics that the cells exhibited during depolarization were explained with, and hence confirm, the bifurcation analysis of the Hodgkin-Huxley model with different sodium and potassium concentrations. Hence, this model is an important tool for understanding the electrical activity of cells during failure of ion concentration homeostasis.

Chapter 6 describes the electrical activity of a large number of synaptically coupled neurons. It was studied how the single cell dynamics determine the emergent macroscopic activity. To allow the investigation of the effects of changes in ion concentrations, a neural mass model that is fully based on physiological parameters was constructed. The firing rate curve of the single cells is used to describe the single cell dynamics. To obtain the population dynamics, the variance of the firing rates and input currents are modeled as well.

The last chapter reflects back on the work performed, and recommendations are given for further research that can improve diagnostics and treatment of patients with hypoxic brain damage.

\section{References}

[1] World Health Organization, "The Atlas of Heart Disease and Stroke", (2004), URL http://www. who.int/cardiovascular_diseases/resources/atlas/en/.

[2] V. Roger, A. Go, and D. Lloyd-Jones, "Heart Disease and Stroke Statistics2011 Update1. About 1. About These Statistics2. American Heart Association's 2020 Impact Goals3. Cardiovascular Diseases4.”, Circulation (2011).

[3] V. E. O'Collins, M. R. Macleod, G. A. Donnan, L. L. Horky, B. H. van der Worp, and D. W. Howells, “1,026 experimental treatments in acute stroke.", Annals of neurology 59, 467-77 (2006).

[4] S. McCann, "Oxidative stress and therapeutic targets for ischaemic stroke (thesis)", Ph.D. thesis, University of Melbourne (2012).

[5] Y. D. Cheng, L. Al-Khoury, and J. a. Zivin, "Neuroprotection for ischemic stroke: two decades of success and failure.", NeuroRx : the journal of the American Society for Experimental NeuroTherapeutics 1, 36-45 (2004).

[6] M.-A. Dronne, E. Grenier, G. Chapuisat, M. Hommel, and J.-P. Boissel, "A modelling approach to explore some hypotheses of the failure of neuroprotective trials in ischemic stroke patients.", Progress in biophysics and molecular biology 97, 60-78 (2008).

[7] G. Z. Feuerstein and J. Chavez, "Translational medicine for stroke drug discovery: the pharmaceutical industry perspective.”, Stroke; a journal of cerebral circulation 40, S121-5 (2009).

[8] K.-A. Hossmann, "Pathophysiology and therapy of experimental stroke.", Cell Mol Neurobiol 26, 1057-1083 (2006). 
[9] L. Vatov, Z. Kizner, E. Ruppin, S. Meilin, T. Manor, and A. Mayevsky, "Modeling brain energy metabolism and function: a multiparametric monitoring approach.", Bull Math Biol 68, 275-291 (2006).

[10] K. Revett, E. Ruppin, S. Goodall, and J. A. Reggia, "Spreading depression in focal ischemia: a computational study.”, J Cereb Blood Flow Metab 18, 998-1007 (1998).

[11] A. V. M. Herz, T. Gollisch, C. K. Machens, and D. Jaeger, "Modeling single-neuron dynamics and computations: a balance of detail and abstraction.", Science 314, 80-85 (2006).

[12] M. C. Tjepkema-Cloostermans, F. B. van Meulen, G. Meinsma, and M. J. van Putten, "A cerebral recovery index (cri) for early prognosis in patients after cardiac arrest.", Crit Care 17, R252 (2013).

[13] G. Deco, V. K. Jirsa, P. A. Robinson, M. Breakspear, and K. Friston, "The dynamic brain: from spiking neurons to neural masses and cortical fields.”, PLoS Comput Biol 4, e1000092 (2008).

[14] I. Bojak, T. F. Oostendorp, A. T. Reid, and R. Ktter, "Connecting mean field models of neural activity to eeg and fmri data.”, Brain Topogr 23, 139-149 (2010).

[15] R. Hindriks and M. J. A. M. van Putten, "Meanfield modeling of propofol-induced changes in spontaneous eeg rhythms.", Neuroimage 60, 2323-2334 (2012).

[16] M. C. Tjepkema-Cloostermans, R. Hindriks, J. Hofmeijer, and M. J. A. M. van Putten, "Generalized periodic discharges after acute cerebral ischemia: Reflection of selective synaptic failure?", Clin Neurophysiol (2013).

[17] A. Aarabi and B. He, "Seizure prediction in hippocampal and neocortical epilepsy using a modelbased approach", Clin Neurophysiol (2014).

[18] O. David, S. J. Kiebel, L. M. Harrison, J. Mattout, J. M. Kilner, and K. J. Friston, "Dynamic causal modeling of evoked responses in eeg and meg.”, Neuroimage 30, 1255-1272 (2006).

[19] D. A. Pinotsis, R. J. Moran, and K. J. Friston, "Dynamic causal modeling with neural fields.", Neuroimage 59, 1261-1274 (2012). 


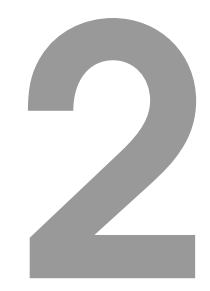

\section{Pathophysiology of ischemic stroke}

Neural tissue needs a constant supply of energy. Reserve energy stores in the brain, in the form of phosphocreatine and glycogen are small and are able to sustain regular cerebral metabolism only for several seconds [1].When blood flow to neural tissue is interrupted, as during ischemia, cellular processes and neural activity quickly fail, eventually resulting in cell death $[2,3]$.

An infarct resulting from ischemia consists of two regions. The core is defined as the region in which there is practically no blood flow, such that energy supply fails. Unless blood flow is restored within minutes (the acute phase), the cells in this core die and cannot be recovered.

The core is surrounded by a penumbra, in which blood flow is reduced, but in which metabolism is partly preserved. Cellular and signaling processes fail depending on the remaining blood flow. The cells in the penumbra are functionally impaired, but can in principle be recovered. In the hours to days after the insult (the subacute phase), the infarct core expands into the penumbra. A cascade of events, involving spreading depolarization, excitotoxicity, inflammation and reactive oxygen species (ROS), may result in delayed neuronal death (see figure 2.1). Therefore, the penumbra is an attractive target for therapeutic interventions.

How the infarct expands, is determined by the dynamic interplay of blood flow, metabolism, neuronal and glial activity and composition of the extracellular space. It is focused on in this chapter, how the energy consumption, neuronal dynamics and ion homeostasis interact in the minutes to hours after the ischemic attack. These pro- 


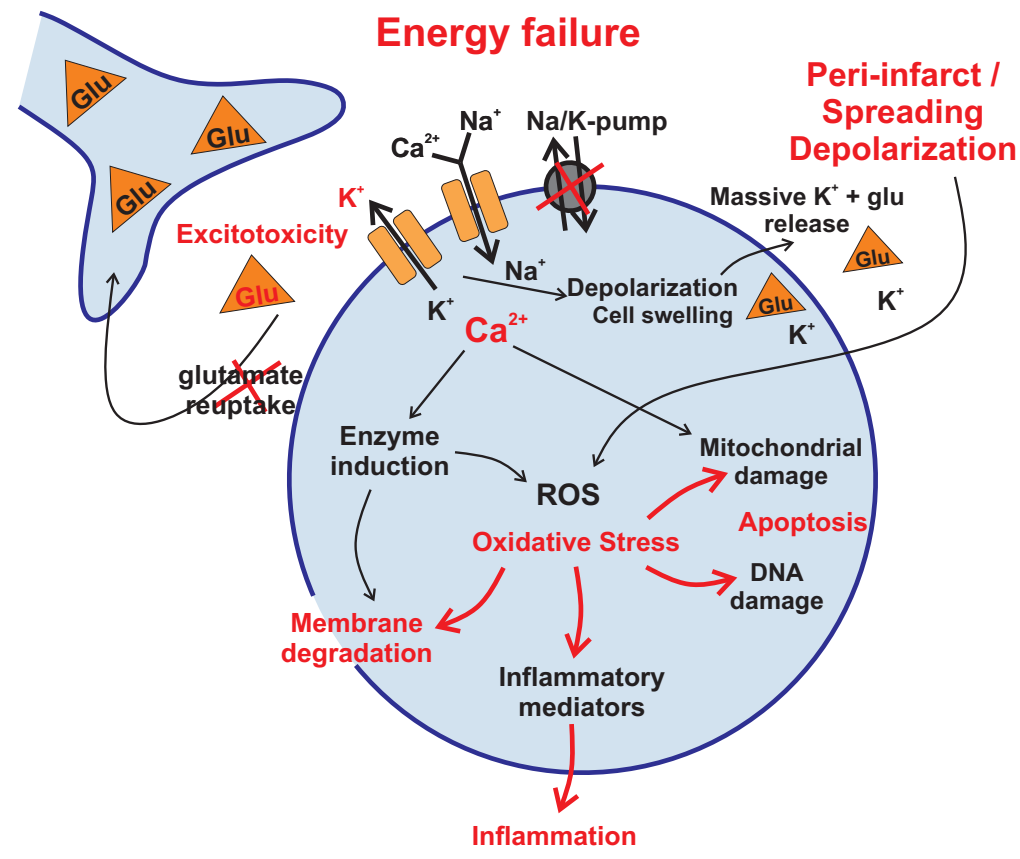

Figure 2.1: Without energy supply, the Na/K-pumps and glutamate reuptake fail. This leads to a build-up of $\mathrm{K}^{+}$and Glu in the extracellular space, which within a minute causes the cells to depolarize, allowing $\mathrm{Ca}^{2+}$ into the cell. Depolarization causes cell swelling and a massive release of $\mathrm{K}^{+}$and $\mathrm{Glu}$, initiating peri-infarct or spreading depolarizations. The intracellular calcium and depolarizations lead indirectly to oxidative stress, membrane degradation and apoptosis, followed by inflammation and damage to the blood brain barrier. 
cesses are important, because they determine three main pathways for cellular damage and death. Cell swelling is induced by osmosis, directly determined by the intraand extracellular ion concentrations. This causes mechanical damage. Furthermore, anoxic depolarization of cells causes an increase in metabolic demand to enable recovery. This generates noxious side products, notably reactive oxygen species [4] and $\mathrm{H}^{+}$[5]. Finally, increased intracellular calcium levels result from neuronal depolarization or failure of calcium transport. These induce mitochondrial damage and apoptosis [3].

The interactions between energy consumption, neural activity and ion homeostasis are discussed, quantitatively, serving as a reference for computational modeling. First, the metabolic budget of the neural unit is described, and the Na/K-pump function is identified as the main expenditure of ATP. It will be shown how the energy consumption of the pump is indirectly determined by the synaptic input and firing rate of the neurons in the tissue. Then an overview is given of which cellular processes fail when energy supply is diminished. It is discussed how first electrical activity is suppressed, thereby preserving energy for ion homeostasis to temporarily prevent neuronal damage. Furthermore, the potassium release and sodium influx in neurons occurring during neural activity is calculated and it is discussed how these in turn influence the neuronal dynamics. Finally, it is discussed how this interaction between ion concentrations and neural activity leads to sudden depolarization of neurons and so-called spreading and peri-infarct depolarization.

\subsection{The neural metabolic unit}

The neuron, the atom of neural functioning, has long been considered the only cell of interest in the brain. The role of glial cells (glue cells) was thought to keep them in place. Now, however, it is known that glial cells have a crucial supporting role, not only mechanical, but also in homeostasis of the extracellular space, signaling to the blood vessels, and in the metabolism of the neurons. A brief overview is given of the metabolism and homeostasis of the so-called neural metabolic unit, consisting of a neuron, synapses, glial cells, extracellular space and a capillary (see figure 2.2).

The molecular interactions in the neural unit will not be detailed here. For a discussion of the metabolic cycles and chemical reactions involved in the generation of ATP and the symbiosis between astrocytes and neurons, the reader is referred to [7-12]. Blood flow regulation and signaling by the neurons and astrocytes to the blood vessels are described in [7, 13-15]. Several computational models of the molecular reactions in metabolism and blood flow signaling have been developed $[1,16-18]$.

The largest part of the energy expenditure of the brain is used for ion homeostasis of the intra- and extracellular space of the neurons. The rest is used for recycling of 


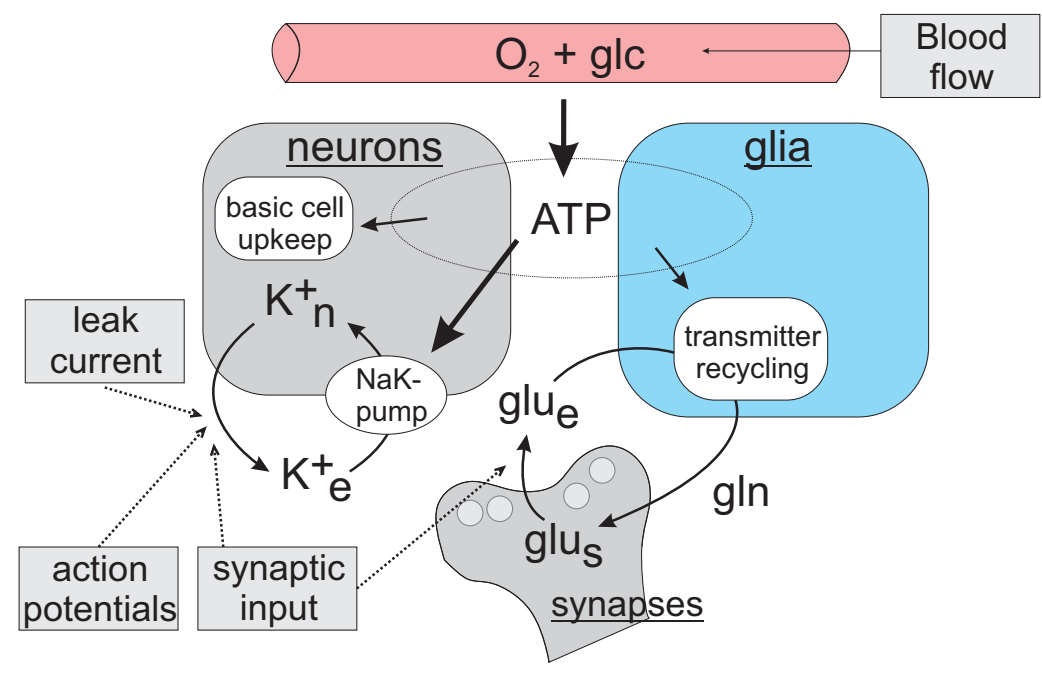

Figure 2.2: Energy balance of the neural unit. The blood flow supplies the unit with oxygen and glucose (Glc). This is used by the mitochondria in the neurons and glia to produce adenosine-triphosphate (ATP). This ATP is mainly consumed by the Na/K-pump, and for a small part by cellular upkeep, such as neurotransmitter recycling [1]. Additionally, the glia cells buffer locally released extracellular potassium, distribute it among a large syncytium of glia, and transport it to the bloodstream [6].

neurotransmitter and a relatively small amount is used on basic cell upkeep, such as protein synthesis and sustaining the mitochondrial membrane voltage [19] (see figure 2.3). Ions flow across the neuronal cell membrane during action potentials and synaptic input, and to a lesser extent leak out during rest. These are transported back by a system of ion pumps and exchangers. Molecular pumps, notably the $\mathrm{Na} / \mathrm{K}-$ pump, use ATP to transport ions, while exchangers use the gradient/energy of one ion species to transport another. When blood flow is interrupted, there is no supply of oxygen and glucose, ATP cannot be generated by the mitochondria, the ion pumps halt, ion homeostasis fails and neural functioning is disrupted.

In conclusion, neurons as well as glia produce ATP from the oxygen and glucose supplied by the blood. This ATP is mainly used for the restoration of the ion gradients following synaptic transmission and action potential generation.

\subsection{Metabolic thresholds of physiological processes}

During ischemia or hypoxia, neural tissue reduces its ATP consumption. This allows the cells to survive several minutes of complete ischemia, or maintain their membrane 


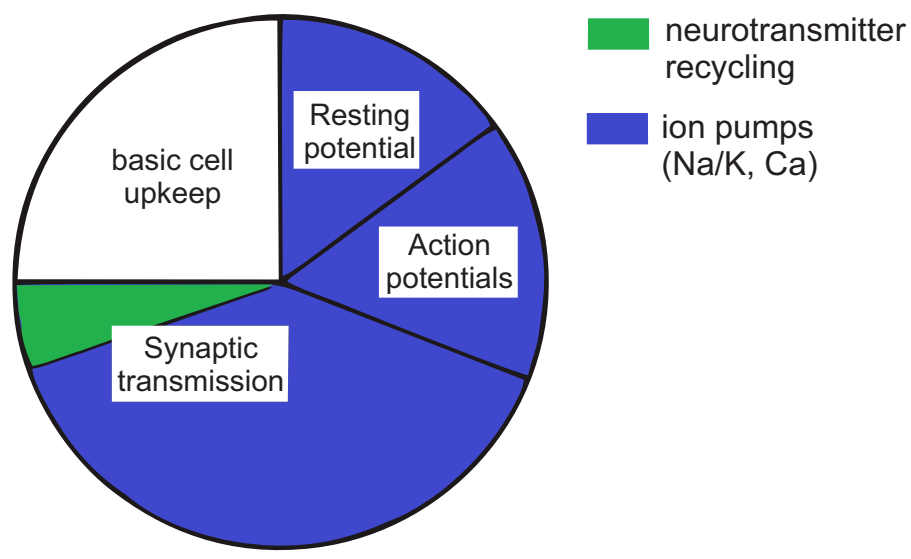

Figure 2.3: Neural energy budget, based on [19, 20]. A small part is spent on basic cellular processes (25\%), while a large part of the budget is used on action potential generation and synaptic transmission. This energy is mainly used by the Na/K-pump. An average firing rate of $4 \mathrm{~Hz}$ was assumed.

potential for longer periods during partial ischemia.* After minutes of complete ischemia, however, cells depolarize and permanent damage occurs soon after.

In the acute phase after stroke (minutes), cellular and electrical signaling processes fail depending on the remaining level of blood flow and concomitant oxygen/glucose levels (figure 2.4). Here, we discuss the order of failure, based on numbers obtained from various experimental measurements reviewed in [3]. These are (qualitatively) representative for the human brain.

- The first process to be affected is protein synthesis, which is reduced by $50 \%$ when blood flow drops from $0.55 \mathrm{~mL} / \mathrm{g} / \mathrm{min}$, and is completely halted at 0.35 $\mathrm{mL} / \mathrm{g} / \mathrm{min}[3]$.

- When perfusion is reduced from 0.35 to $0.3 \mathrm{~mL} / \mathrm{g} / \mathrm{min}$, anaerobic glycolysis is stimulated. The blood supplies a surplus of glucose compared to the amount of oxygen, which is used to maintain ATP production. This doubles the glucose consumption of the tissue [3].

Here we calculate the energy supplied to the tissue by the blood flow. Arterial blood contains typically $5.5 \mathrm{mM}$ glucose (Glc), and $0.2 \mathrm{~mL} \mathrm{O}_{2} / \mathrm{mL}$ blood, which equals $9 \mathrm{mM} \mathrm{O}_{2}\left(1 \mathrm{mmol} \mathrm{O} \mathrm{O}_{2}\right.$ equals $22.4 \mathrm{~mL}$ at standard temperature and pressure). If

${ }^{*}$ Lutz and others performed fascinating work on hypoxia resistant animals. In certain carps, for example, the mechanisms that reduce ATP consumption are perfected such that these animals are able to survive up to months without oxygen. Acidification from the little remaining anaerobic metabolism is prevented by sweating out alcohol [21]. 


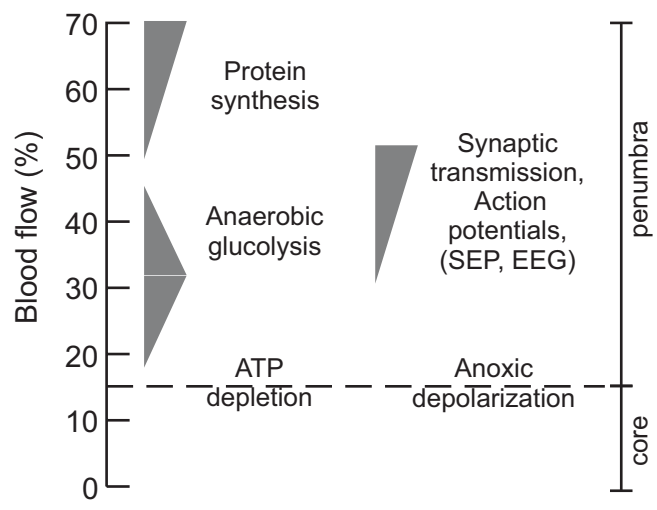

Figure 2.4: Blood flow thresholds for failure of metabolic and electrophysiological processes. Protein synthesis fails first, followed by synaptic and electrical activity, reflected in the somatosensory evoked potential and EEG. Upon further reduction of blood flow, the cells depolarize. The core is the region where blood flow is insufficient to sustain ATP levels, resulting in anoxic depolarization. Based on [3].

sufficient oxygen is present, 36 ATP molecules are produced using 1 Glc and 6 $\mathrm{O}_{2}$. Anaerobic glycolysis is much less efficient, yielding only 2 ATPs per glucose molecule [22].

Assuming all oxygen and glucose is extracted from the blood, $1 \mathrm{~mL}$ blood provides $54 \mu \mathrm{mol}$ ATP $(1.5 \mu \mathrm{mol}$ Glc) through aerobic respiration, and, when this is insufficient for the tissues needs, another $8 \mu \mathrm{mol}$ of ATP can be provided through

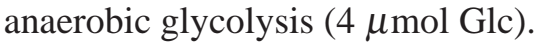

The energy consumption of the whole brain is approximately $20 \mu \mathrm{mol} \mathrm{ATP} / \mathrm{g} / \mathrm{min}$ [19], which corresponds to the aerobic energy supplied by $0.37 \mathrm{~mL}$ blood $/ \mathrm{g} / \mathrm{min}$, or total energy supplied by $0.32 \mathrm{~mL} / \mathrm{g} / \mathrm{min}$. This is indeed approximately the observed range in which glucose consumption is increased.

- Below $0.25 \mathrm{~mL} / \mathrm{g} / \mathrm{min}$, neural activity is reduced, which is reflected in the EEG and somatic evoked potential (SEP) [3].

Neural activity is reduced in two ways. After ischemia/hypoxia, synaptic transmission is one of the first processes to fail [23]. This process is not well-understood, but suppression of presynaptic calcium influx plays an important role [24], as well as adenosine, a breakdown product of ATP, blocking synapses after depolarization [25]. Furthermore, ATP-sensitive potassium channels are activated, which hyperpolarize the membrane potential. These are activated by an increase in ADP/ATP ratio, allowing them to sense depletion of ATP early [26]. As discussed previously, the cessation of neural activity greatly reduces the energy consumption. 
- Between 0.25 and $0.10 \mathrm{~mL} / \mathrm{g} / \mathrm{min}$, ATP concentrations gradually drop from close to $100 \%$ to 0 [3].

- Below $0.15 \mathrm{~mL} / \mathrm{g} / \mathrm{min}$, the energy supply is insufficient to maintain the membrane potential and neurons depolarize. They release potassium and glutamate [3], and receive a large calcium influx.

In summary, depending on the reduction in blood flow and concomitant oxygen/glucose deprivation, cellular and electrical signaling processes seize one by one. In part, these are safety mechanisms that reduce the metabolic demand of the tissue, in order to preserve the $\mathrm{Na} / \mathrm{K}$-pump function to maintain the neuronal membrane potential.

\subsection{The influence of neuronal activity on ion homeostasis and neural metabolism}

When neuronal firing rates increase, efflux of potassium and influx of sodium increases. It is shown how the metabolism and dynamics of the ion concentrations depend on the neuronal firing rate. The potassium efflux from a neuron into the extracellular space is calculated during rest and during an action potential, as well as the amount of ATP consumed to transport the ions back. The potassium fluxes from the neurons are calculated here for rodent cortical tissue, from the bottom-up estimations of ATP consumption by pyramidal cells of Attwell et al. [19, 20]. Their estimates are rough, since several values with large experimental uncertainties had to be used, but the corresponding energy consumption is similar to that observed experimentally [8].

During an action potential, sodium flows in and depolarizes the membrane, followed by an efflux of potassium that repolarizes the membrane. From the membrane area, capacitance and time course of the action potential of a pyramidal cell, the amount of intracellular potassium ions that are exchanged with intracellular sodium during an action potential (AP) are estimated as $3.6 \times 10^{8} \mathrm{~K}^{+}$ions/AP. Attwell et al. estimated the leak currents from the input conductance and resting membrane voltage as $1.0 \times 10^{9} \mathrm{~K}^{+}$ions /neuron /s. Furthermore, an action potential induces presynaptic calcium influx in the synapses, which subsequently release glutamate. Glutamate induces postsynaptic calcium and sodium influx, through NMDA and non-NMDA receptors. Calcium and glutamate are transported using the sodium gradient. Resulting from the restoration of ion concentrations and uptake of glutamate, an amount of $3.3 \times 10^{8}$ ATP/AP is consumed by the $\mathrm{Na} / \mathrm{K}$-pump. (Assuming on average 2000 synapses per neuron release a vesicle each AP) These processes also indirectly release approximately triple this amount, $1.0 \times 10^{9} / \mathrm{AP}$, of $\mathrm{K}^{+}$ions from the neurons and glia. Two-thirds of these are pumped back by the Na/K-pump, and the other third drifts back to compensate the net pump current [19]. 
From these potassium effluxes the rate at which the extracellular concentration rises is calculated here: $0.75 \mathrm{mM} / \mathrm{s}$ due to the leak current and $1.0 \mathrm{mM} / \mathrm{s} / \mathrm{Hz}$ due to action potential generation and synaptic transmission. (Assuming $9 \times 10^{7}$ pyramidal cells $/ \mathrm{cm}^{3}$ and an extracellular space of $20 \%$ of the tissue volume.) The physiological extracellular concentration of potassium is typically $4.0 \mathrm{mM}$. This concentration would double within seconds during normal neuronal activity $(4 \mathrm{~Hz}$ average firing rate), if no homeostasis mechanisms were present.

These numbers illustrate that potassium efflux and sodium influx drastically increases with the neuronal firing rate. This in turn increases the extracellular potassium concentration and intracellular sodium concentrations [27], innervating the $\mathrm{Na} / \mathrm{K}$ pumps $[28,29]$ and increase neural energy consumption. In the next section it is discussed what influence a rise in extracellular potassium has on neuronal action potential generation.

\subsection{The role of ion concentrations in neuronal activity}

Ionic homeostasis enables proper electrophysiological functioning of the neurons. A neurons soma is enclosed by a semi-permeable membrane, that functions as a capacitor $C_{m}$, whose voltage dynamics are determined by an input current from the dendrite and the ionic transmembrane currents $I_{x}$ :

$$
C_{m} \frac{\mathrm{d} V}{\mathrm{~d} t}=-\sum I_{x}+I_{\text {input }} .
$$

The summation is over the ion species $x$ for which the membrane is permeable, notably $\mathrm{Na}, \mathrm{K}$ and $\mathrm{Cl}$. The ionic currents can be derived from the Nernst-Planck equation describing ion fluxes due to diffusion and drift on an axis perpendicular to the cells membrane. This results in the Goldman-Hodgkin-Katz (GHK) current equation. This current is induced by two effects, diffusion due to the ion gradients and drift due to the voltage over the membrane. For each ion species, a reversal or Nernst potential $E_{x}$ exists, at which drift balances diffusion. This potential is a function of the intraand extracellular concentrations, $C_{i n}$ and $C_{\text {out }}$ :

$$
E_{x}=\frac{k T}{z q} \log \frac{C_{\text {in }}}{C_{\text {out }}},
$$

where $k$ is the Boltzmann constant, $T$ the temperature, $q$ the elementary charge and $z$ the valence of the respective ion species $\mathrm{x}$.

A simple approximation for the transmembrane ionic currents, used in the HodgkinHuxley model [30], is one that is linear with the voltage, describing the ionic conductance as a voltage source and resistor with conductance $g_{x}$ in series: ${ }^{\dagger}$

$$
I_{x}=g_{x}(t)\left(V-E_{x}\right) .
$$

\footnotetext{
${ }^{\dagger}$ In contrast to what is stated throughout literature on physiology, for the $\mathrm{HH}$ current to be an accurate
} 
Each ionic current drives the membrane voltage towards its corresponding Nernst potential. The non-linearity of the dynamics, i.e. the ability to generate action potentials, is described by the dependence of $g_{x}$ on voltage gated channels in the membrane.

Neglecting the effects of pump currents and other ions, the resting membrane potential $V_{r}$ is a weighted sum of the $E_{N a}, E_{K}$ and $E_{C l}$ :

$$
V_{r}=\frac{\sum g_{x} E_{x}}{\sum g_{x}} .
$$

Due to the relatively large permeability to potassium, a neurons resting membrane voltage is close to $E_{k}$. Furthermore, the largest relative changes in concentration are observed in $\left[\mathrm{K}^{+}\right]_{e}$, since the extracellular potassium concentration is relatively low (table 2.2) and the extracellular space is small. Therefore, of all ion concentrations, extracellular potassium has the most pronounced effect on the neuronal activity.

Another way in which the ion concentrations affect the membrane voltage is through ion pump activity. The current generated by the Na/K-pump, for example, lowers the membrane voltage. To enable homeostasis, this pump rate is sensitive to the extracellular potassium concentration $[28,29]$.

In conclusion, the membrane voltage dynamics depend on the intra- and extracellular concentrations of sodium, potassium and chlorine, mainly through their Nernst potentials (equation 2.2). These determine the resting voltage, as well as the membrane currents. The corresponding membrane dynamics will be considered in more detail in chapters 3-6.

\subsection{Spreading Depolarization}

Spreading depolarization (SD) is a phenomenon that emerges from the dynamics of metabolism, neuronal activity and the extracellular homeostasis mechanisms. SD is a slowly propagating wave $(\mathrm{mm} / \mathrm{min})$ of neuronal depolarization, characterized by shifts in the intra- and extracellular ion concentrations and depressed electrical activity [6], as shown in figure 2.5 .

SDs occur around ischemic infarcts (peri-infarct depolarizations, PID) as well traumatic brain injuries. They are also the substrate of the migraine aura, which propagates over the cortex, temporarily disabling brain functions [34]. When occurring as a migraine aura, SD is not harmful for the tissue, since sufficient energy is

approximation of the GHK current, the intra- and extracellular concentrations must be approximately equal. It is not sufficient if they are in the same order of magnitude [31, 32]. However, the non-linearity of the current does not qualitatively affect the dynamics of action potential generation, nor does it change the qualitative dynamics of the ion concentrations themselves [33]. Therefore the HH equations will be used in this thesis, since they are well-known and simple. 

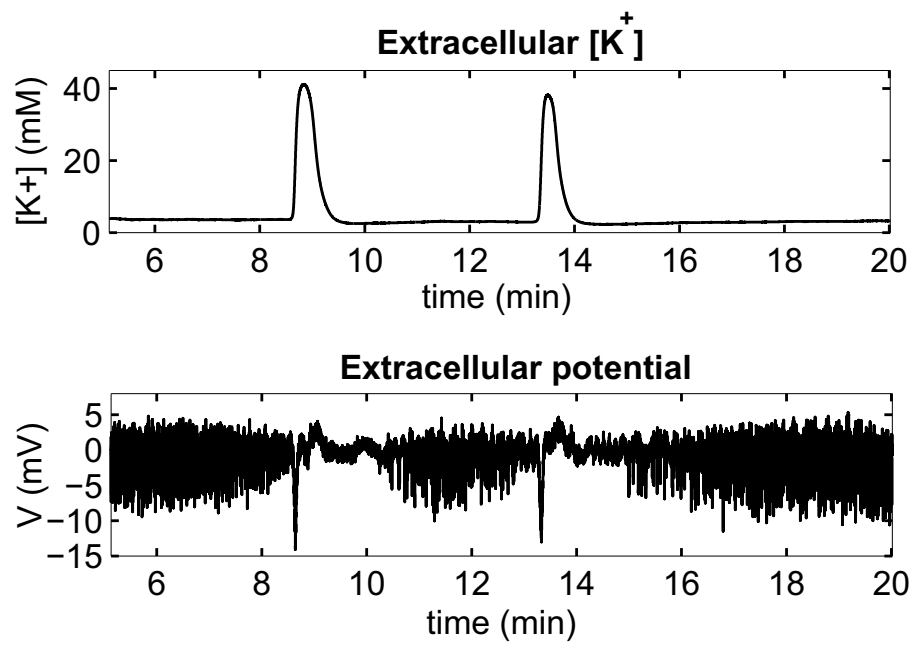

Figure 2.5: Two consecutive spreading depressions, in rat cortex in vivo. Experimentally induced by application of $\mathrm{KCl}$ with a cotton ball in a burr hole, approximately a $\mathrm{cm}$ from the measurement site. The extracellular potassium concentration and the extracellular potential (high pass filtered) were obtained from a double barreled potassium sensitive electrode. At the onset of an SD, a rapid increase of $\left[\mathrm{K}^{+}\right]_{e}$ can be observed, signaling the depolarization of the neurons. Simultaneously, the spikes in the extracellular potential, reflecting neuronal activity, are depressed. $\left[\mathrm{K}^{+}\right]_{e}$ is restored in approximately a minute, while the neuronal activity recovers after approximately 2 minutes. 
supplied by increased blood flow to restore the ion concentrations within minutes. After ischemic stroke, however, its occurrence is correlated with infarct growth and poor neurological outcome [35].

In the penumbra, where energy supply is already critical, the recovery from SD adds further stress to the metabolic system. Other pathways causing damage are the calcium influx induced by prolonged depolarization and the large amounts of reactive oxygen species that are generated by neurons during and following SD [4]. These oxidatively damage the neuronal membrane and induce apoptosis (see figure 2.1). On the other hand, SD may have a neuroprotective role. It signals for an increased blood flow and furthermore, tissue preconditioned with SD has been found to be more resistant to hypoxia ([36] and references therein).

Experimentally, SD can be induced by various noxious stimuli, e.g. ischemia, intense electrical stimulation or application of $\mathrm{K}+$ /glutamate. In vivo, spreading depression is most likely initiated by a rise in extracellular potassium. Potassium and glutamate excite neurons, increasing the firing rate, and thereby stimulate their own release. To prevent $\left[\mathrm{K}^{+}\right]_{e}$ and $[\mathrm{Glu}]$ from rising beyond control, glial cells remove released potassium and glutamate from the extracellular space [37]. However, when insufficient ATP is available for neuronal and glial $\mathrm{Na} / \mathrm{K}$-pump activity, or the neuronal activity is pathologically high, the removal mechanisms cannot balance the release. This results in a sudden rise in extracellular potassium and glutamate, depolarizing the neurons. This depolarization can propagate by diffusion of extracellular potassium and glutamate through the extracellular space. Other propagation mechanisms, for example through neuronal gapjunctions, have been hypothesized as well [38].

Summarizing, SD is a process during which the dynamics of the ion concentrations and the neuronal membrane voltage strongly interact, resulting in large efflux of potassium and glutamate and depolarization of neurons. Its occurrence has been shown in several studies to correlate with infarct growth and poor neurological outcome.

\subsection{Conclusion}

The metabolic energy budget of the neural unit was discussed, as well as which processes fail first during a metabolic deficiency. The metabolic demand increases greatly with the neuronal firing rate, largely mediated by the ATP consumption of the ion pumps restoring the ion gradients. Increased firing rates, or impairment of the ion homeostasis mechanisms lead to changes in intra- and extracellular ion concentrations, most notably extracellular potassium. This in turn increases the neuronal excitability. When metabolic demand is not balanced by sufficient supply of oxygen and glucose from the blood, cellular and signaling processes fail at different thresh- 
olds of blood flow. Maintenance of the neuronal membrane voltage is the last process to be preserved, to prevent potassium efflux, neuronal depolarization and calcium influx, resulting in cell swelling and cellular damage. The dynamics of these processes and their interactions determine, in part, the progression of an ischemic infarct.

\section{References}

[1] M. Cloutier, F. B. Bolger, J. P. Lowry, and P. Wellstead, "An integrative dynamic model of brain energy metabolism using in vivo neurochemical measurements.”, J Comput Neurosci 27, 391-414 (2009).

[2] K. Krnjević, "Electrophysiology of cerebral ischemia.”, Neuropharmacology 55, 319-33 (2008).

[3] K.-A. Hossmann, "Pathophysiology and therapy of experimental stroke.", Cell Mol Neurobiol 26, 1057-1083 (2006).

[4] A. Viggiano, E. Viggiano, I. Valentino, M. Monda, A. Viggiano, and B. De Luca, "Cortical spreading depression affects reactive oxygen species production.”, Brain research 1368, 11-8 (2011).

[5] W. A. Mutch and A. J. Hansen, "Extracellular ph changes during spreading depression and cerebral ischemia: mechanisms of brain ph regulation.”, J Cereb Blood Flow Metab 4, 17-27 (1984).

[6] G. G. Somjen, Ions in the Brain - Normal Function, Seizures, and Stroke (Oxford University Press) (2004).

[7] P. K. Shetty, F. Galeffi, and D. A. Turner, "Cellular links between neuronal activity and energy homeostasis.", Front Pharmacol 3, 43 (2012).

[8] F. Hyder, A. B. Patel, A. Gjedde, D. L. Rothman, K. L. Behar, and R. G. Shulman, "Neuronalglial glucose oxidation and glutamatergic-gabaergic function.", J Cereb Blood Flow Metab 26, 865-877 (2006).

[9] A. Gjedde, Brain energetics: integration of molecular and cellular processes, chapter 4, 351 353 (Springer) (2007).

[10] A. Gjedde, S. Marrett, and M. Vafaee, "Oxidative and nonoxidative metabolism of excited neurons and astrocytes.”, J Cereb Blood Flow Metab 22, 1-14 (2002).

[11] S. Mangia, F. Giove, I. Tkc, N. K. Logothetis, P.-G. Henry, C. A. Olman, B. Maraviglia, F. Di Salle, and K. U? urbil, "Metabolic and hemodynamic events after changes in neuronal activity: current hypotheses, theoretical predictions and in vivo nmr experimental findings.', J Cereb Blood Flow Metab 29, 441-463 (2009).

[12] L. Pellerin and P. J. Magistretti, "Glutamate uptake into astrocytes stimulates aerobic glycolysis: a mechanism coupling neuronal activity to glucose utilization.", Proc Natl Acad Sci U S A 91 , 10625-10629 (1994).

[13] D. Attwell, A. M. Buchan, S. Charpak, M. Lauritzen, B. A. Macvicar, and E. A. Newman, "Glial and neuronal control of brain blood flow.", Nature 468, 232-243 (2010). 
[14] M. Zonta, M. C. Angulo, S. Gobbo, B. Rosengarten, K.-A. Hossmann, T. Pozzan, and G. Carmignoto, "Neuron-to-astrocyte signaling is central to the dynamic control of brain microcirculation.", Nat Neurosci 6, 43-50 (2003).

[15] C. Iadecola and M. Nedergaard, "Glial regulation of the cerebral microvasculature.", Nat Neurosci 10, 1369-1376 (2007).

[16] A. Aubert and R. Costalat, "A model of the coupling between brain electrical activity, metabolism, and hemodynamics: application to the interpretation of functional neuroimaging.", Neuroimage 17, 1162-1181 (2002).

[17] L. Vatov, Z. Kizner, E. Ruppin, S. Meilin, T. Manor, and A. Mayevsky, "Modeling brain energy metabolism and function: a multiparametric monitoring approach.”, Bull Math Biol 68, 275-291 (2006).

[18] H. Farr and T. David, "Models of neurovascular coupling via potassium and eet signalling.", J Theor Biol 286, 13-23 (2011).

[19] D. Attwell and S. B. Laughlin, "An energy budget for signaling in the grey matter of the brain.", J Cereb Blood Flow Metab 21, 1133-1145 (2001).

[20] C. Howarth, P. Gleeson, and D. Attwell, "Updated energy budgets for neural computation in the neocortex and cerebellum.", J Cereb Blood Flow Metab 32, 1222-1232 (2012).

[21] P. W. Hochachka and P. L. Lutz, "Mechanism, origin, and evolution of anoxia tolerance in animals.”, Comp Biochem Physiol B Biochem Mol Biol 130, 435-459 (2001).

[22] W. Boron and E. Boulpaep, Medical Physiology (Elsevier Health Sciences) (2008).

[23] J. Hofmeijer and M. J. A. M. van Putten, "Ischemic cerebral damage: an appraisal of synaptic failure.", Stroke 43, 607-615 (2012).

[24] J. N. Young and G. G. Somjen, "Suppression of presynaptic calcium currents by hypoxia in hippocampal tissue slices.”, Brain research 573, 70-6 (1992).

[25] B. E. Lindquist and C. W. Shuttleworth, "Adenosine receptor activation is responsible for prolonged depression of synaptic transmission after spreading depolarization in brain slices.", Neuroscience 223, 365-376 (2012).

[26] N. Inagaki and S. Seino, "ATP-sensitive potassium channels: structures, functions, and pathophysiology", The Japanese journal of physiology 48, 397-412 (1998).

[27] W. Walz, "Potassium homeostasis in the brain at the organ and cell level", in Non-Neuronal Cells of the Nervous System: Function and Dysfunction, edited by L. Hertz, volume Volume 31, 604 (Elsevier) (2003), URL http://www.sciencedirect.com/science/article/pii/S1569255803310276.

[28] L. P. Endresen, K. Hall, J. S. Hø ye, and J. Myrheim, "A theory for the membrane potential of living cells.”, European biophysics journal : EBJ 29, 90-103 (2000).

[29] P. Luger, "Electrogenic properties of the na,k-pump.”, Prog Clin Biol Res 273, 217-224 (1988).

[30] A. L. Hodgkin and A. F. Huxley, "A quantitative description of membrane current and its application to conduction and excitation in nerve.", J Physiol 117, 500-544 (1952). 
[31] M. A. Herrera-Valdez, "Membranes with the same ion channel populations but different excitabilities", PLoS ONE 7, e34636 (2012).

[32] J. Keener and J. Sneyd, Mathematical Physiology, chapter 3, 2nd edition (Springer) (2009).

[33] N. Hübel, E. Schöll, and M. A. Dahlem, "Bistable dynamics of ion homeostasis in ion-based neuron models", ArXiv e-prints (2013).

[34] M. Lauritzen, J. P. Dreier, M. Fabricius, J. a. Hartings, R. Graf, and A. J. Strong, "Clinical relevance of cortical spreading depression in neurological disorders: migraine, malignant stroke, subarachnoid and intracranial hemorrhage, and traumatic brain injury.”, Journal of cerebral blood flow and metabolism : official journal of the International Society of Cerebral Blood Flow and Metabolism 31, 17-35 (2011).

[35] H. Nakamura, A. J. Strong, C. Dohmen, O. W. Sakowitz, S. Vollmar, M. Su, L. Kracht, P. Hashemi, R. Bhatia, T. Yoshimine, J. P. Dreier, A. K. Dunn, and R. Graf, "Spreading depolarizations cycle around and enlarge focal ischaemic brain lesions.", Brain 133, 1994-2006 (2010).

[36] H. M. Gniel and R. L. Martin, "Cortical spreading depression-induced preconditioning in mouse neocortex is lamina specific.”, J Neurophysiol 109, 2923-2936 (2013).

[37] G. G. Somjen, "Mechanisms of spreading depression and hypoxic spreading depression-like depolarization.", Physiol Rev 81, 1065-1096 (2001).

[38] B. E. Shapiro, "Osmotic forces and gap junctions in spreading depression: a computational model.”, J Comput Neurosci 10, 99-120 (2001). 


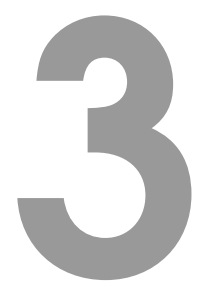

\title{
Neural Dynamics during Anoxia and the "Wave of Death" *
}

\begin{abstract}
Recent experiments in rats have shown the occurrence of a high amplitude slow brain wave in the EEG approximately 1 minute after decapitation, with a duration of 5-15 s (van Rijn et al, PLoS One 6 , e16514, 2011) that was presumed to signify the death of brain neurons. We present a computational model of a single neuron and its intra- and extracellular ion concentrations, which shows the physiological mechanism for this observation. The wave is caused by membrane potential oscillations, that occur after the cessation of activity of the sodium-potassium pumps has led to an excess of extracellular potassium. These oscillations can be described by the Hodgkin-Huxley equations for the sodium and potassium channels, and result in a sudden change in mean membrane voltage. In combination with a high-pass filter, this sudden depolarization leads to a wave in the EEG. We discuss that this process is not necessarily irreversible.
\end{abstract}

*Published as: BJ Zandt, B ten Haken, JG van Dijk, MJAM van Putten (2011) Neural Dynamics during Anoxia and the Wave of Death. PLoS ONE 6(7): e22127. doi:10.1371/journal.pone.0022127 


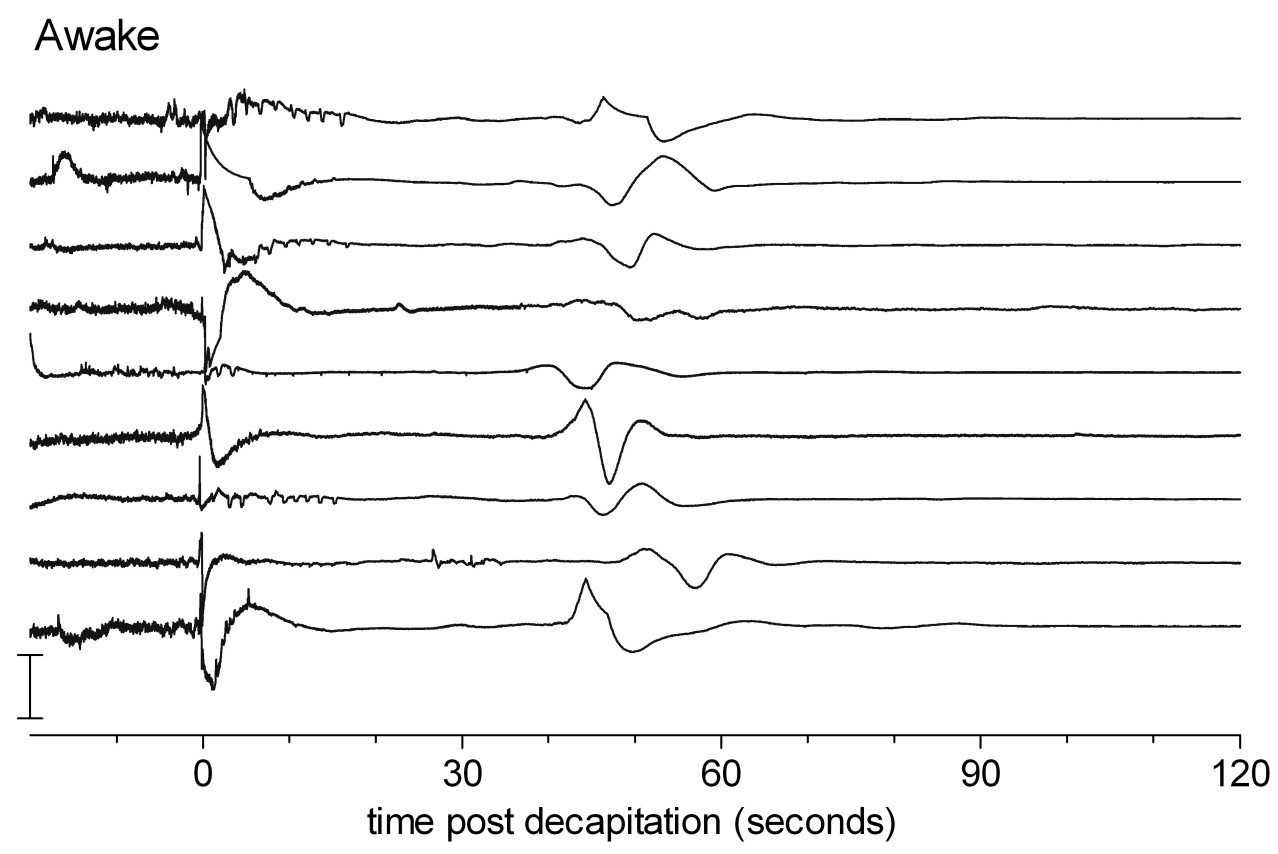

Figure 3.1: EEGs recorded in 9 animals after decapitation. Note the large slow wave around $50 \mathrm{~s}$ after decapitation. Similar experiments were performed in an anesthetized group of animals, where the wave appeared at a slightly later instant, approximately $80 \mathrm{~s}$. The changes in amplitude at $t=0$ are movement artifacts due to the decapitation. Figure from [3].

\subsection{Introduction}

Oxygen and glucose deprivation has almost immediate effects on brain function, typically causing symptoms in approximately 5-7 seconds. This dysfunction is also reflected in the electroencephalogram (EEG), generally consisting of an increase in slow wave activity and finally in the cessation of activity. These phenomena are a direct consequence of synaptic failure of pyramidal cells [1], reflecting the high metabolic demand of synaptic transmission [2].

Recent findings in rats, decapitated to study whether this is a humane method of euthanasia in awake animals, indeed showed disappearance of the EEG signal after approximately $15-20 \mathrm{~s}$. After half a minute of electrocerebral silence, however, a slow wave with a duration of approximately 5-15 seconds appeared (Figure 3.1). It was suggested that this wave might reflect the synchronous death of brain neurons [3] and was therefore named the "Wave of Death".

Similar experiments were performed by Swaab and Boer in 1972 [4]. The EEG survival time was of the same order as the observations of van Rijn et al [3]: after approximately $7 \mathrm{~s}$ the EEG flattened to become iso-electric after $20 \mathrm{~s}$. Recordings 
did not last longer than that, however, which may explain why the "Wave of Death" was not detected in these experiments.

Van Rijn et al. [3] speculated that the wave might be due to a simultaneous and massive loss of resting membrane potential, caused by the oxygen-glucose deprivation (OGD) following decapitation. Indeed, plenty of (experimental) literature exists showing that hypoxia causes membrane depolarization. Siemkowicz and Hansen [5], for instance, induced complete cerebral ischemia in rats for ten minutes. During and after this period they recorded an EEG and measured the extracellular potential and extracellular ion concentrations. A rapid deflection of the extracellular potential occurred typically 1-2 minutes after the onset of ischemia, accompanied by a sudden rise in extracellular potassium. Unfortunately, EEG activity during the ischemic episode was not described and it is unknown whether a similar wave in the EEG occurred here. Another example is the work of Dzhala et al., who perfused rat brains in vivo with an anoxic-aglycemic solution and measured the transmembrane potential of a pyramidal cell. Approximately eight minutes after the onset of the induced ischemia, they observed a rapid depolarization of the cell membrane [6]. Depolarization is also observed in computational models. For example, Kager et al. modeled neuronal dynamics and ion concentrations and show that an increased concentration of potassium in the neuronal environment can cause fast membrane depolarizations. Depolarization also takes place in their simulations when the ion pump rates are lowered and a neuron is stimulated by injecting current for a few $100 \mathrm{~ms}$ [7, 8].

In this chapter we present a minimal biophysical, single-cell model. Using HodgkinHuxley dynamics to describe the voltage-dependent ion channel dynamics, including oxygen/glucose dependent ion pumps, we show that severe oxygen-glucose deprivation results in a sudden depolarization of the membrane voltage. Subsequent modeling of the EEG results in a macroscopic wave, as observed by van Rijn et al. [3]. Finally we discuss that this wave does not reflect irreversible damage and hence not death.

\subsection{Methods}

\subsubsection{Biophysical model}

A biophysically realistic neuron is modeled using Hodgkin-Huxley dynamics of sodium and potassium channels combined with leak currents. The model includes the dynamics of the extra- and intracellular ion concentrations, which change significantly when homeostasis cannot be maintained by neurons and glia. Ion pump fluxes are incorporated to model this homeostasis. Our model is based on the equations by Cressman et al [9-11], who studied the effects of the extracellular ion concentrations in the generation of epileptic seizures. 
The model consists of an intracellular and an extracellular compartment separated by a semi-permeable cell membrane. This membrane contains a fast transient sodium channel, a delayed rectifier potassium channel and a leak for sodium, potassium and chlorine. The dynamics of the membrane voltage, $V$, are described with the HodgkinHuxley equations:

$$
C \frac{\mathrm{dV}}{\mathrm{dt}}=-I_{\mathrm{Na}}\left(m_{\infty}(V), h, V-E_{N a}\right)-I_{\mathrm{K}}\left(n, V-E_{K}\right)-I_{\mathrm{Cl}}\left(V-E_{C l}\right)
$$

with $C$ the membrane capacitance and $I_{\mathrm{Na}}, I_{\mathrm{K}}, I_{\mathrm{Cl}}$ the total sodium, potassium and chloride currents. The Nernst potential for each ion species is indicated with $E_{\mathrm{x}}$ and given by $E_{x}=\frac{k T}{q z_{x}} \cdot \log \left([x]_{e} /[x]_{i}\right)$, with $k$ the Boltzmann constant, $T$ the absolute temperature, $z_{x}$ the valency of the ion, $[x]_{i}$ and $[x]_{e}$ the intra- and extracellular concentrations and $\mathrm{x}=\mathrm{Na}, \mathrm{K}, \mathrm{Cl}$. The fraction of activated sodium channels, $m_{\infty}(V)^{3}$ is due to its fast dynamics assumed to depend instantaneously on the membrane voltage. $h$ is the fraction of inactivated sodium channels and is a variable in our model. $n$ is the fraction of activated potassium channels and is also a variable. The calcium gated current from the Cressman model is not implemented, because it does not qualitatively alter the behavior of interest here. We write for the total sodium, potassium and chloride currents

$$
\begin{aligned}
I_{\mathrm{Na}} & =g_{\mathrm{Na}} m_{\infty}(V)^{3} h(t)\left[V-E_{\mathrm{Na}}(t)\right]+g_{\mathrm{NaL}}\left[V-E_{\mathrm{Na}}(t)\right] \\
I_{\mathrm{K}} & =g_{\mathrm{K}} n(t)^{4}\left[V-E_{K}(t)\right]+g_{\mathrm{KL}}\left[V-E_{\mathrm{KL}}(t)\right] \\
I_{\mathrm{Cl}} & =g_{\mathrm{ClL}}\left[V-E_{\mathrm{Cl}}(t)\right],
\end{aligned}
$$

respectively. The maximum ion conductances for the gated currents are denoted with $g_{x}$ and for the leak currents with $g_{x L}$.

The gating variables $m_{\infty}(V), n$ and $h$ are modeled as [11]:

$$
\begin{aligned}
m_{\infty}(V) & =\alpha_{m}(V) /\left(\alpha_{m}(V)+\beta_{m}(V)\right) \\
\alpha_{m}(V) & =(V+30 \mathrm{mV}) /[(1-\exp (-(\mathrm{V}+30 \mathrm{mV}) / 10 \mathrm{mV})) \cdot 10 \mathrm{mV}] \\
\beta_{m}(V) & =4 \cdot \exp (-(\mathrm{V}+55 \mathrm{mV}) /(18 \mathrm{mV})) \\
\frac{\mathrm{dq}}{\mathrm{dt}} & =\phi\left[\alpha_{q}(V)(1-q)-\beta_{q}(V) q\right], \quad q=n, h \\
\alpha_{n}(V) & =(V+34 \mathrm{mV}) /[(1-\exp (-(\mathrm{V}+34 \mathrm{mV}) / 10 \mathrm{mV})) \cdot 100 \mathrm{mV}] \\
\beta_{n}(V) & =0.125 \cdot \exp (-(\mathrm{V}+44 \mathrm{mV}) /(80 \mathrm{mV})) \\
\alpha_{h}(V) & =0.07 \cdot \exp (-(\mathrm{V}+44 \mathrm{mV}) /(20 \mathrm{mV})) \\
\beta_{h}(V) & =1 /(1+\exp (-(\mathrm{V}+14 \mathrm{mV}) /(10 \mathrm{mV}))),
\end{aligned}
$$

where $\phi$ is the time constant of the channels. When the ion concentrations, on which the Nernst potentials depend, are assumed to be constant, equation sets 3.1 to 3.3 can be used to model the dynamical behavior of a single neuron. 
In order to calculate changes in ion concentrations in the model, equations are added that integrate the ion fluxes into and out of the two compartments. During physiological conditions, the concentrations are given by [11]:

$$
\begin{aligned}
\frac{\mathrm{d}[\mathrm{Na}]_{\mathrm{i}}}{\mathrm{dt}} & =\frac{A}{V F}\left(-I_{N a}-3 I_{p}\right) & \frac{\mathrm{d}[\mathrm{Na}]_{\mathrm{e}}}{\mathrm{dt}} & =-\frac{\beta A}{V F}\left(-I_{N a}-3 I_{p}\right) \\
\frac{\mathrm{d}[\mathrm{K}]_{\mathrm{i}}}{\mathrm{dt}} & =\frac{A}{V F}\left(-I_{K}+2 I_{p}\right) & \frac{\mathrm{d}[\mathrm{K}]_{\mathrm{e}}}{\mathrm{dt}} & =-\frac{\beta A}{V F}\left(-I_{K}+2 I_{p}\right)-I_{g}-I_{d} \\
\frac{\mathrm{d}[\mathrm{Cl}]_{\mathrm{i}}}{\mathrm{dt}} & =0 & \frac{\mathrm{d}[\mathrm{Cl}]_{\mathrm{e}}}{\mathrm{dt}} & =0,
\end{aligned}
$$

with $\mathrm{A}$ and $\mathrm{V}$ respectively the surface area and volume of the cell, $\mathrm{F}$ the Faraday constant and $\beta$ the ratio of the intra- and extracellular volumes. $I_{p}$ denotes a sodiumpotassium pump current (in $\mu \mathrm{A} / \mathrm{cm}^{2}$ ) which depends sigmoidally on the intracellular sodium concentration and the extracellular potassium concentration. The total amount of sodium is preserved in this model, but the extracellular potassium can be buffered by glial cells $\left(I_{g}\right)$ and can diffuse from and into the blood $\left(I_{d}\right)$. Furthermore, the chlorine concentrations are assumed to remain constant under normal conditions, without specifying the mechanism for this. The approximation that the efflux of potassium equals the influx of sodium made by Cressman et al. in order to reduce the number of variables is not made here.

The pump, glial and diffusion currents are modeled as [11]:

$$
\begin{aligned}
I_{p} & =\left(\frac{\rho_{p}}{1+\exp \left(\left(25 \mathrm{mM}-[\mathrm{Na}]_{\mathrm{i}}\right) /(3 \mathrm{mM})\right)}\right) \times \\
& \times\left(\frac{1}{1+\exp \left(\left(5.5 \mathrm{mM}-[\mathrm{K}]_{\mathrm{e}}\right) /(1 \mathrm{mM})\right)}\right) \\
I_{g} & =\frac{G}{1+\exp \left(\left(18 \mathrm{mM}-[\mathrm{K}]_{\mathrm{e}}\right) /(2.5 \mathrm{mM})\right)} \\
I_{d} & =\varepsilon\left([\mathrm{K}]_{\mathrm{e}}-\mathrm{k}_{\infty}\right)
\end{aligned}
$$

Here $\rho_{p}$ scales the pump rate, $G$ the glial buffering rate, $\varepsilon$ is the time constant of diffusion and $k_{\infty}$ the concentration of potassium in the blood. Note that $I_{g}$ and $I_{d}$ do not have the dimension of current, but that of rate of change of concentration $(\mathrm{mM} / \mathrm{s})$.

\subsubsection{Numerical implementation}

Equation sets 3.1 to 3.5 completely describe our model. The resting state of this system is calculated, with the parameters shown in table 3.1. The equations were solved with a solver for stiff ordinary differential equations (ode23 routine, Matlab, the Mathworks). The simulation code is available from ModelDB [12], accession number 139266. Table 3.2 shows the results of this calculation, which are used as 
starting point for the simulation of oxygen and glucose deprivation. It was verified that the model behaves as expected under normal circumstances: in rest the membrane potential and the sodium and potassium concentrations are in the physiological range. Furthermore the neuron responds with a single action potential when a short current pulse is applied and spikes periodically when a current of $1.5 \mu \mathrm{A} / \mathrm{cm}^{2}$ or more is injected.

To simulate the anoxia and aglycemia, we set both the pump current and the uptake of $\mathrm{K}^{+}$ions by the glial cells to zero as well as diffusion of $\mathrm{K}^{+}$to the blood. Furthermore the chlorine concentrations are no longer assumed to stay constant. This changes the equations for the concentration dynamics, Eqns 3.4, into:

$$
\frac{\mathrm{d}[\mathrm{x}]_{\mathrm{e}}}{\mathrm{dt}}=-\frac{\beta A}{V z_{x} F} I_{x}, \quad \frac{\mathrm{d}[\mathrm{x}]_{\mathrm{i}}}{\mathrm{dt}}=-\frac{A}{V z_{x} F} I_{x}, \quad \text { for } \mathrm{x}=\mathrm{K}, \mathrm{Na}, \mathrm{Cl}
$$

\subsection{Results}

In the case of a normally functioning neuronal unit, which maintains homeostasis, the model reaches a steady state with a membrane potential and ionic concentrations in physiological ranges (Table 3.2). Figure 3.2 shows the result of our simulation of oxygen and glucose deprivation using this steady state as a starting point. Initially, over the course of half a minute, the membrane voltage rises by approximately 0.7 $\mathrm{mV} / \mathrm{s}$. This is due to the efflux of potassium, which causes a rise in $\left[\mathrm{K}^{+}\right]_{\mathrm{e}}$ and correspondingly in $E_{K}$. The rise in $E_{K}$ is only partially compensated by the fall of $E_{\mathrm{Na}}$, caused by the influx of sodium ions.

At $\mathrm{t}=28.7 \mathrm{~s}$, the resting membrane voltage reaches the excitation threshold, such that the resting state of the cell loses stability and the cell starts to generate action potentials (spikes) with an initial frequency of $10 \mathrm{~Hz}$, increasing to $500 \mathrm{~Hz}$ in a $7 \mathrm{~s}$ period.

Each spike temporarily opens the potassium channels and transiently increases the efflux of potassium. The resulting increase of the extracellular potassium concentration in turn increases the mean membrane voltage and spiking frequency, forming a positive feedback loop. As a result, the mean membrane potential (Figure 3.2, left panel) steeply rises from -50 to $-20 \mathrm{mV}$ in the last 2 seconds of this oscillation period. During this $2 \mathrm{~s}$ period, the amplitude of the action potential spikes decreases to zero, after which the neuron obtains a stable resting state again. In this state, however, the neuron is no longer excitable, due to the so-called depolarization block, i.e. the permanent inactivation of the sodium channels. After the neuron stops spiking, the leak currents cause the difference between the Nernst potentials of sodium and potassium to slowly vanish over the course of a minute. Due to the small chlorine leak the Nernst potentials and membrane voltage eventually reach $-20 \mathrm{mV}$ after about ten minutes (not shown). 

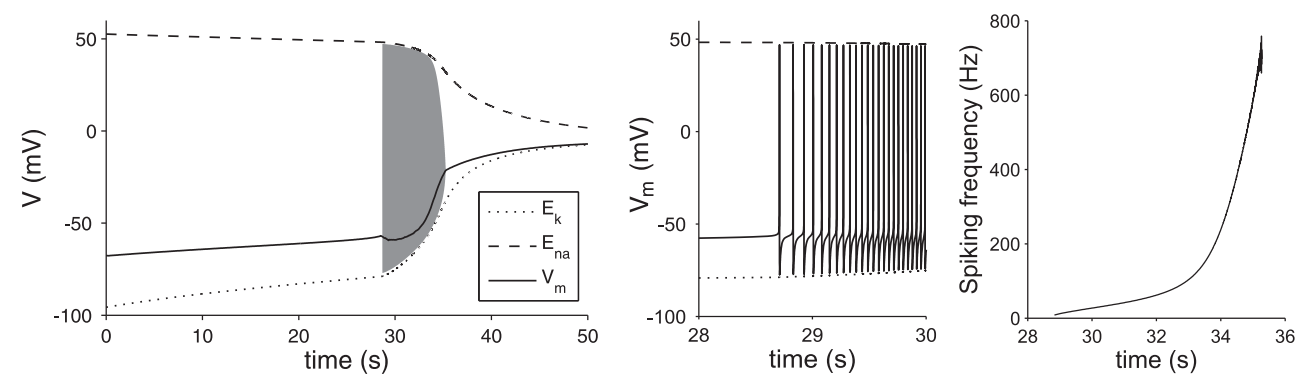

Figure 3.2: Membrane dynamics during oxygen-glucose deprivation. In the left panel the membrane dynamics are shown that occur after the onset of OGD (solid line). The dashed and dotted lines show the progressive loss of ion gradients. When after a gradual rise the membrane potential reaches the excitation threshold, this subsequently results in spiking of the membrane voltage according to Eqns 3.1 and 3.2 (gray region, not resolved). The black line shows the average membrane potential during the spiking (averaged over $300 \mathrm{~ms}$ ). After approximately 7 seconds of oscillations, the cell comes to rest again, with a resulting $V_{\mathrm{m}} \approx$ $-20 \mathrm{mV}$. The middle panel shows a close up of the start of spiking activity, the right panel shows the instantaneous firing rate.

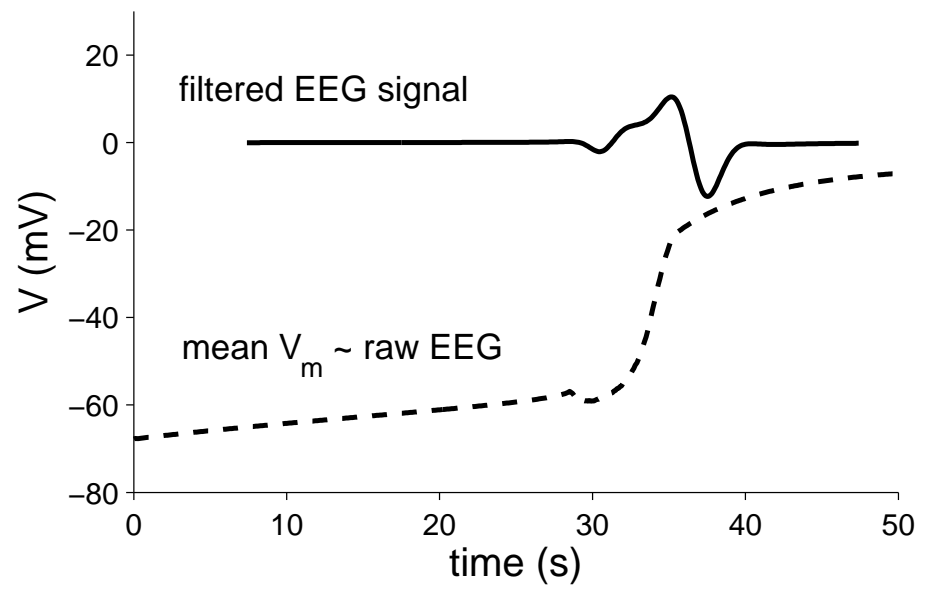

Figure 3.3: Mean membrane potential and simulated EEG signal. Shown are (dashed line) the simulated membrane potential averaged over $300 \mathrm{~ms}$ and in (solid line, a.u.) the signal that results after applying a high-pass filter $\left(2^{\text {nd }}\right.$ order Butterworth filter, cut-off at 0.1 $H z)$ 
In order to compare the simulated single cell behavior with the EEG observed by van Rijn et al. [3], we proceed as follows. The contribution of a single cell to the (raw) EEG is roughly proportional to its membrane potential [13]. Modeling the EEG realistically usually requires a large scale simulation with many neurons, because the behavior of a cell depends heavily on its interaction with other neurons. The present situation provides an exception, however, because synaptic transmission has stopped and neurons receive no direct input. As a result, their dynamics can be accurately described with a single cell model; the EEG of an ensemble of cells can be calculated by simply summing the contributions of individual neurons. Assuming that many neurons behave approximately the same as the modeled neuron, but with some small shift in time, the resulting raw EEG is proportional to the mean membrane potential (Figure 3.3, dashed line). For simplicity, a flat distribution of $300 \mathrm{~ms}$ wide was chosen, but varying the shape and width of this distribution hardly changes the resulting EEG. High-pass filtering the resulting potential with a cut-off at $0.1 \mathrm{~Hz}$ replicates the filter characteristics of the filter used by van Rijn et al. [3]. This results in the solid curve shown in Figure 3.3, similar to the reported "Wave of Death" (cf Figure 3.1 with solid curve in Figure 3.3).

\subsection{Discussion}

Dynamic phenomena that occur during hypoxia and the way they are reflected in the EEG are only partially understood. Measurements of extreme cases showing clear features in the EEG present an opportunity to gain insight in the relation with the underlying physiology. Such an extreme case is decapitation, in which the supply of energy to the entire brain is halted almost instantaneously. This causes the EEG to become flat after several seconds, but also results in a large amplitude wave approximately a minute after decapitation. Van Rijn et al. suggest that this wave "ultimately reflects brain death" [3], but also state that further research on the physiology of brain function during this process is needed.

We modeled the membrane voltage dynamics of a single neuron with a sodium and a potassium channel and leak currents, together with the corresponding changes in the intra- and extracellular ion concentrations. This model can explain the physiological origin of the wave. When a sodium-potassium pump, glial buffering and diffusion of potassium are incorporated to model homeostasis, the model shows regular behavior and has a resting state where all variables obtain values in their physiological ranges. After shutting down the energy supply, the membrane initially depolarizes slowly with a slope of approximately $0.7 \mathrm{mV} / \mathrm{s}$, until it reaches the excitation threshold, around $-58 \mathrm{mV}$. Now spiking starts, resulting in an increase in the potassium current with a concomitant reduction in the potassium Nernst potential and membrane voltage. Positive feedback between the increasing firing rate and potassium 
efflux causes a sudden depolarization of the membrane voltage $(30 \mathrm{mV}$ in 2 seconds), resulting in the membrane depolarization curve, displayed as a dashed line in Figure 3.3. In combination with a high-pass filter, the simulated membrane voltage results in a wave in the EEG as observed by van Rijn et al. (Figure 3.3, solid line). This behavior was also observed in the in vivo measurements in rats by Siemkowicz and Hansen [5], who also measured a rapid depolarization accompanied with an increase of extracellular potassium, typically 1-2 minutes after the onset of ischemia.

While modeling the effects of decapitation, an instantaneous cessation of the sodium-potassium pump, glial buffering and diffusion of potassium to the blood was assumed. The last assumption is very reasonable, because arterial pressure vanishes after decapitation, larger vessels are drained and blood flow through the capillaries will stop. The (remaining) blood volume is relatively small and the ion concentrations in the blood will therefore quickly equilibrate with the tissue. However, a complete stop of all active ion transport will not take place directly after decapitation. Some reserves of metabolic substrates and ATP are still left in the tissue. In human brain tissue for example, these reserves can support a maximum of one minute of normal metabolism [14], but less if no oxygen is available. Such effects do not disqualify the general behavior of the model, as they will only result in a delay in the onset of depolarization, in line with the observations by van Rijn et al. Siemkowicz and Hansen [5] hypothesized that the transition from a slow to a fast rise of extracellular potassium and the corresponding depolarization is the result of depletion of these energy reserves; they hypothesized that the pumps are initially still partially fueled by anaerobic glycolysis until the glucose reserve is depleted and the ion pumps stop, causing a large efflux of potassium. We show here, however, that this is not the case and that the transition results from the Hodgkin-Huxley dynamics of the voltage dependent channels in the cell membrane.

A single neuron model was used to calculate an EEG. Although usually the network properties of neurons are essential for the EEG, we argued that a single neuron approach is realistic because synaptic transmission ceases quickly during anoxia and neurons therefore no longer receive input. Such an early cessation of transmission during hypoxia is due to failure of neurotransmitter release, presumably caused by failure of the presynaptic calcium channels [15]. Although the postsynaptic response is still intact, for example the response of the neuron to glutamate [16], neurotransmitters are no longer released and transmission is halted. The absence of significant EEG power after about 20 seconds post decapitation as observed by van Rijn et al. most likely results from this failure of synaptic transmission.

The depolarization wave was observed during a relatively short period of $\sim$ $10-15 \mathrm{~s}$. As the extracellular currents generated by a single pyramidal neuron are of the order of $\mathrm{pA}$, much too small to generate a measurable scalp potential, a very large number of cortical neurons must simultaneously depolarize after decapitation. Such 
a synchronization has indeed been measured by Aitken et al., who induced depolarization in hippocampal slices, by either injecting $\mathrm{KCl}$ or halting the oxygen supply, to simulate spreading depression and hypoxia respectively. 1-3 minutes after the onset of hypoxia, small foci of depolarization appeared and spread with a speed of approximately $0.1 \mathrm{~mm} / \mathrm{s}$ [17]. The propagation of depolarization is hypothesized to be caused by diffusion of $\mathrm{K}^{+}$or glutamate or by interaction through gap junctions [8]. The observed speed of propagation in these slices is too slow to account for the depolarization of all cells in a whole (rat) brain in a few seconds. However, in the case of decapitation the supply of both oxygen and glucose is stopped simultaneously in the entire brain, so it is likely that many of these foci are formed simultaneously by cells of a single type with approximately the same properties. Other, fast, non-synaptic mechanisms, ephaptic transmission and electrical field effects [18], may play a role in the synchronization as well.

The absence of significant EEG power after the depolarization wave is caused by the depolarization block, which by no means implies irreversibility or cell death. Physiological cell dynamics can in principle be restored by resupplying the ion pumps with ATP. Siemkowicz and Hansen [5] observed the return of EEG activity 15 minutes after a period of isoelectricity caused by complete ischemia. High levels of intracellular calcium and cell swelling, associated with hypoxic depolarization, can be highly damaging to neurons; still this damage typically takes place over a period of hours [19]. Irreversible functional damage due to oxygen and glucose deprivation most likely occurs from damage to synapses, rather than from cell death itself [2]. In line with this perhaps surprising result, cells from neocortical slices from adult human brain obtained several hours postmortem, can survive for weeks in vitro [20]. We therefore reject the claim in the paper by van Rijn et al. [3] that this particular phenomenon can be used clinically to determine brain death. In fact, this wave does not imply death, neither of neurons nor of individuals.

In summary, our simulations and the data presented from experimental physiology show that the "Wave of Death" reflects the sudden change in membrane potential due to anoxic depolarization, that is a direct result of the Hodgkin-Huxley dynamics and ion concentrations. Although the wave is indeed a typical signature of the final membrane voltage changes of neurons suffering from severe oxygen and glucose deprivation, it is not a biomarker for irreversibility (e.g. Siemkowicz and Hansen [5]). Anoxic/hypoxic depolarizations are a well-known phenomenon in experimental physiology and can be simulated with our physiologically plausible minimal model. Therefore, a more appropriate name for this phenomenon is "cerebral anoxic depolarization". 


\section{A Parameters}

Table 3.1 shows the parameter values used in the simulation. Values are taken from [11]. (For the correct units, refer to [11] rather than [9]). We changed the definition of $\rho_{p}$ to yield a pump current density rather than a rate of change of concentration. The ratio of intra- and extracellular volume, $\beta$, is chosen as 2 rather than 7 . This is calculated from measurements by Mazel et al. that show that the extracellular space constitutes $20 \%$ of the total tissue volume in rat brain [21] and by assuming the rest of the tissue consists of approximately the same volume of glia as neurons.

Table 3.1: Overview of the parameters used in the simulations.

\begin{tabular}{|c|c|c|c|}
\hline variable & value & units & description \\
\hline$\overline{C_{m}}$ & 1.0 & $\mu \mathrm{F} / \mathrm{cm}^{2}$ & specific membrane capacitance \\
\hline$g_{n a}$ & 100 & $\mathrm{mS} / \mathrm{cm}^{2}$ & sodium channel conductance \\
\hline$g_{\text {naL }}$ & 0.0175 & $\mathrm{mS} / \mathrm{cm}^{2}$ & sodium leak conductance \\
\hline$g_{k}$ & 40 & $\mathrm{mS} / \mathrm{cm}^{2}$ & potassium channel conductance \\
\hline$g_{k L}$ & 0.05 & $\mathrm{mS} / \mathrm{cm}^{2}$ & potassium leak conductance \\
\hline$g_{c l L}$ & 0.05 & $\mathrm{mS} / \mathrm{cm}^{2}$ & chlorine leak conductance \\
\hline$\phi$ & 3 & $\mathrm{~ms}^{-1}$ & time constant of gating variables \\
\hline$A / V F$ & 0.044 & $\frac{\mathrm{mM}}{\mathrm{s}} /\left(\frac{\mu \mathrm{A}}{\mathrm{cm}^{2}}\right)$ & conversion factor current to concentration \\
\hline$\beta$ & 2.0 & & ratio intra- / extracellular volume \\
\hline$\rho_{p}$ & 28.1 & $\mu \mathrm{A} / \mathrm{cm}^{2}$ & NaK-Pump rate \\
\hline$G$ & 66 & $\mathrm{mM} / \mathrm{s}$ & glial buffering rate of $\mathrm{K}^{+}$ \\
\hline$\varepsilon$ & 1.3 & $\mathrm{~s}^{-1}$ & diffusion rate \\
\hline$k_{\infty}$ & 4.0 & $\mathrm{mM}$ & concentration $\mathrm{K}^{+}$in blood \\
\hline$T$ & 310 & $\mathrm{~K}$ & absolute temperature \\
\hline
\end{tabular}

Table 3.2: Overview of the variables in the steady state.

\begin{tabular}{lll} 
variable & $\begin{array}{l}\text { steady state } \\
\text { value }\end{array}$ & units \\
\hline$V_{\mathrm{m}}$ & -68 & $\mathrm{mV}$ \\
{$\left[\mathrm{K}^{+}\right]_{\mathrm{i}}$} & 139 & $\mathrm{mmol}$ \\
{$\left[\mathrm{K}^{+}\right]_{\mathrm{e}}$} & 3.8 & $\mathrm{mmol}$ \\
{$\left[\mathrm{Na}^{+}\right]_{\mathrm{i}}$} & 20 & $\mathrm{mmol}$ \\
{$\left[\mathrm{Na}^{+}\right]_{\mathrm{e}}$} & 144 & $\mathrm{mmol}$ \\
{$\left[\mathrm{Cl}^{-}\right]_{\mathrm{i}}$} & 6.0 & $\mathrm{mmol}$ \\
{$\left[\mathrm{Cl}^{-}\right]_{\mathrm{e}}$} & 130 & $\mathrm{mmol}$ \\
\hline \hline
\end{tabular}




\section{References}

[1] M. J. A. M. van Putten and M. H. P. M. van Putten, "Uncommon EEG burst-suppression in severe postanoxic encephalopathy.”, Clin Neurophysiol 121, 1213-1219 (2010).

[2] H. Bolay, Y. Grsoy-Ozdemir, Y. Sara, R. Onur, A. Can, and T. Dalkara, "Persistent defect in transmitter release and synapsin phosphorylation in cerebral cortex after transient moderate ischemic injury.", Stroke 33, 1369-1375 (2002).

[3] C. M. van Rijn, H. Krijnen, S. Menting-Hermeling, and A. M. L. Coenen, "Decapitation in rats: latency to unconsciousness and the 'wave of death'.', PLoS One 6, e16514 (2011).

[4] D. F. Swaab and K. Boer, "The presence of biologically labile compounds during ischemia and their relationship to the eeg in rat cerebral cortex and hypothalamus.", J Neurochem 19, 2843 2853 (1972).

[5] E. Siemkowicz and A. J. Hansen, "Brain extracellular ion composition and eeg activity following 10 minutes ischemia in normo- and hyperglycemic rats.", Stroke 12, 236-240 (1981).

[6] V. Dzhala, I. Khalilov, Y. Ben-Ari, and R. Khazipov, "Neuronal mechanisms of the anoxia-induced network oscillations in the rat hippocampus in vitro.", J Physiol 536, 521-531 (2001).

[7] H. Kager, W. J. Wadman, and G. G. Somjen, "Simulated seizures and spreading depression in a neuron model incorporating interstitial space and ion concentrations.", J Neurophysiol 84, 495$512(2000)$.

[8] G. G. Somjen, "Mechanisms of spreading depression and hypoxic spreading depression-like depolarization.”, Physiol Rev 81, 1065-1096 (2001).

[9] J. R. Cressman, G. Ullah, J. Ziburkus, S. J. Schiff, and E. Barreto, “The influence of sodium and potassium dynamics on excitability, seizures, and the stability of persistent states: I. single neuron dynamics.", J Comput Neurosci 26, 159-170 (2009).

[10] J. R. Cressman, G. Ullah, J. Ziburkus, S. J. Schiff, and E. Barreto, "Erratum to: The influence of sodium and potassium dynamics on excitability, seizures, and the stability of persistent states: I. single neuron dynamics.", J Comput Neurosci (2011).

[11] E. Barreto and J. R. Cressman, "Ion concentration dynamics as a mechanism for neuronal bursting”, (2011), journal of Biological Physics.

[12] M. L. Hines, T. Morse, M. Migliore, N. T. Carnevale, and G. M. Shepherd, "Modeldb: A database to support computational neuroscience.", J Comput Neurosci 17, 7-11 (2004).

[13] R. Plonsey and R. C. Barr, Bioelectricity, 3rd edition (Springer) (2007).

[14] A. Gjedde, Brain energetics: integration of molecular and cellular processes, chapter 4, 351 353 (Springer) (2007).

[15] G. G. Somjen, Ions in the Brain - Normal Function, Seizures, and Stroke, chapter 18 (Oxford University Press) (2004).

[16] M.-K. Sun, H. Xu, and D. L. Alkon, "Pharmacological protection of synaptic function, spatial learning, and memory from transient hypoxia in rats.", J Pharmacol Exp Ther 300, 408-416 (2002). 
[17] P. G. Aitken, G. C. Tombaugh, D. A. Turner, and G. G. Somjen, "Similar propagation of sd and hypoxic sd-like depolarization in rat hippocampus recorded optically and electrically.", J Neurophysiol 80, 1514-1521 (1998).

[18] J. G. Jefferys, "Nonsynaptic modulation of neuronal activity in the brain: electric currents and extracellular ions.”, Physiol Rev 75, 689-723 (1995).

[19] P. Lipton, “Ischemic cell death in brain neurons.”, Physiol Rev 79, 1431-1568 (1999).

[20] R. W. H. Verwer, E. J. G. Dubelaar, W. T. J. M. C. Hermens, and D. F. Swaab, "Tissue cultures from adult human postmortem subcortical brain areas.”, J Cell Mol Med 6, 429-432 (2002).

[21] T. Mazel, Z. Simonov, and E. Sykov, "Diffusion heterogeneity and anisotropy in rat hippocampus.”, Neuroreport 9, 1299-1304 (1998). 


\title{
4 \\ Diffusing substances during spreading depolarization: analytical expressions for propagation speed, triggering and concentration time-courses *
}

\begin{abstract}
Spreading depolarization (SD) is an important phenomenon in stroke and migraine. The processes underlying the propagation of $\mathrm{SD}$, however, are still poorly understood and an elementary model that is both physiological and quantitative is lacking. We show that during the onset and propagation of SD the concentration time courses of excitatory substances such as potassium and glutamate can be described with a reaction-diffusion equation. This equation contains four physiological parameters: a concentration threshold for excitation, a release rate, a removal rate and an effective diffusion constant. Solving this equation yields expressions for the propagation velocity, concentration time courses and the minimum stimulus that can trigger SD. This framework allows for analyzing experimental results in terms of these four parameters. The derived time courses are validated with measurements of potassium in rat brain tissue.
\end{abstract}

*Published as: BJ Zandt, B ten Haken, MJAM van Putten (2013) Diffusing substances during spreading depolarization: analytical expressions for propagation speed, triggering and concentration time-courses. The Journal of Neuroscience, 33(14):59155923 


\subsection{Introduction}

A spreading depression, or depolarization (SD), is a slow wave which progresses through neural tissue, depolarizing neurons and depressing EEG activity, as was discovered by Leão [1]. Spreading depressions are encountered in various pathologies in which metabolism and/or ionic homeostasis are compromised. SD's can be observed after stroke as peri-infarct depolarizations (PID) enlarging the damaged tissue area [2]. SD's can occur during global hypoxia and are the substrate of a migraineous aura as well [3].

Typically, a slowly progressing $(2-10 \mathrm{~mm} / \mathrm{min})$ wave is reported, in which neurons depolarize and ion concentrations in both the intracellular and extracellular space change drastically. After tens of seconds, the ion concentrations are gradually restored, neurons repolarize and function returns. However, under unfavorable circumstances no recovery of the ion concentrations takes place, hence the neurons no longer function and eventually die.

Experimentally, SD can be induced by any stimulus that excites or depolarizes the membrane potential. Notable examples are the application of glutamate or potassium, electrical stimulation and hypoxia. Once triggered, an SD wave propagates independent of the amplitude or kind of the stimulus. For reviews see [3-6].

Even after 50 years of research, it is still not clear what the exact mechanisms of propagation of SD are. It is also not clear whether different forms of SD, e.g. hypoxic versus normoxic, have different propagation mechanisms. We hope to contribute to solving the puzzle by providing analytical expressions that describe the dynamics of diffusible substances, such as potassium and glutamate, that lead to propagation and initiation of SD. To the best of our knowledge, such expressions have not been derived using physiological parameters.

Traditionally, extracellular diffusion of glutamate or potassium was hypothesized as the propagation mechanism for SD. Various experiments, however, suggest alternative mechanisms. In normoxic SD, for example, extracellular potassium does not increase until after the extracellular voltage changes [7]. Therefore, diffusion through the opening of gap junctions is now thought to play a major role in SD propagation, c.f. $[4,8,9]$.

We show that regardless of which substance or pathway of diffusion is most prominent, the contribution of diffusible substances to the propagation of SD can be described with the same general expressions.

We will discuss how the process of release and diffusion of an excitatory substance such as glutamate or potassium during the onset of SD can be described. This allows the construction of a reaction-diffusion equation, that can solved analytically. This results in expressions for both the propagation speed and the stimulus strength needed to elicit SD, which indicates how susceptible tissue is to SD. The solutions that correspond to an SD wave are compared with measurements from literature and 
are shown to describe these very well. Furthermore, we give examples of how the results of experimental measurements and numerical simulations can be analyzed with these expressions.

\subsection{Methods}

In essence, the onset of an SD wave is a reaction-diffusion (RD) process, which can be described by a reaction-diffusion equation. These equations express the changes in the concentration of a particular substance due to its generation, removal and diffusion [10]. The term diffusion is used here in the broadest sense. It can be either passive diffusion through Brownian motion, or any other form of movement or transport through the tissue, as long as the substance is moved along its concentration gradient.

Reaction-diffusion models for SD with varying amounts of physiological detail have been proposed [11-14]. However, the complexity of these models does not allow for analytical solutions. In a personal communication to Grafstein [15], Hodgkin and Huxley already solved a reaction-diffusion equation for SD, calculating the concentration of extracellular potassium, with a cubic polynomial for the reaction rate. This approach produces an analytical solution for the propagation velocity of SD. We will use a similar approach here, but use parameters and a reaction rate that are closer related to the underlying physiology.

The case in which a cubic polynomial is used as reaction rate in SD is discussed in $[16,17]$ and is more generally known as the Schlögl model [18]. SD is mathematically analogous to other phenomena, such as a flame on a matchstick [19]. Therefore, the solutions that we will obtain for our choice of the reaction rate are a specific case of the general results for initiation [20] and propagation [21] of waves in an excitable medium [19].

\subsubsection{Concentration dynamics of an excitatory substance}

We will discuss how the concentration of a single excitatory substance can be described, starting with the processes involved in the reaction. How multiple substances can interact during SD will be discussed briefly in section 4.4.3.

Since in experiments the application of extracellular potassium is often used to elicit SD and several concentration time courses are available from experiments in literature as well, we will use this as an example throughout this chapter. Note, however, that our approach is general, and is not limited to this particular choice.

In physiological conditions, the concentration $C$ of extracellular potassium is approximately $3 \mathrm{mM}$. This is the resting concentration $C_{0}$, at which there is an equilibrium between the efflux of potassium into the extracellular space from the neu- 


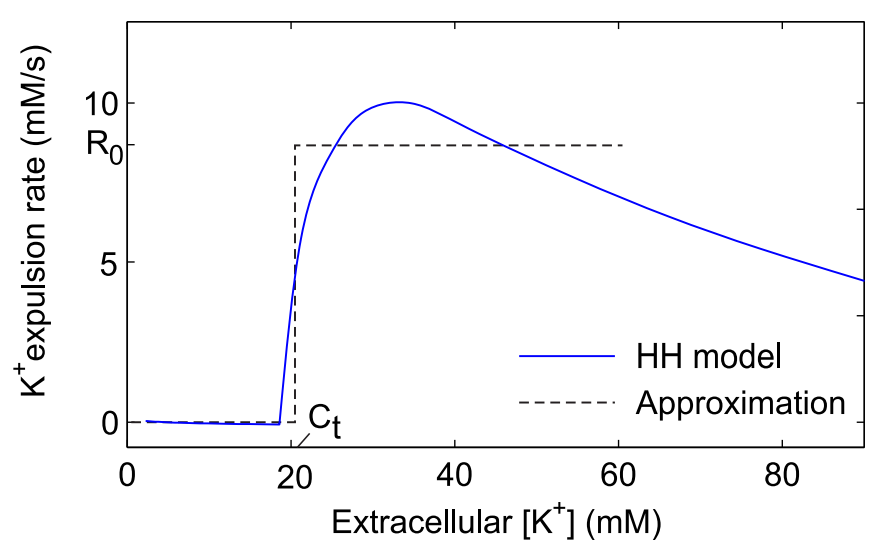

Figure 4.1: The average potassium efflux as a function of $\left[K^{+}\right]_{e}$ from a single HodgkinHuxley type neuron without input. Ion concentrations were kept constant at resting levels in the simulations and only the extracellular potassium concentration was changed. The time averaged leak and gated membrane currents were then summed. A critical concentration $C_{t}$ can be observed at approximately $20 \mathrm{mM}$ after which the neuron starts spiking and releases large amounts of potassium. The dotted line shows a step function approximation.

rons and glia and the influx of potassium through $\mathrm{Na} / \mathrm{K}$-pump activity. Physiological homeostasis mechanisms, e.g. increase of $\mathrm{Na} / \mathrm{K}$-pump activity and diffusion to the bloodstream, work to return the concentration to $C_{0}$ after changes from its equilibrium value, for example resulting from increased neural activity.

For simplicity we assume that the concentration relaxes exponentially to this equilibrium, so that the rate $R_{r}(C)$ at which the concentration decreases is proportional to the concentration difference from equilibrium:

$$
R_{r}(C)=\frac{d C}{d t}=G \cdot\left(C-C_{0}\right)
$$

where $G$ is the constant of proportionality. Since we will mainly deal with excess concentrations, we will refer to $G$ as the removal constant.

For pathologically high concentrations, however, another effect takes place due to the excitatory effect of extracellular potassium. As an illustration, the time averaged potassium release from a single, unstimulated, neuron is calculated with a model adapted from Chapter 3. Figure 4.1 shows this potassium efflux as a function of extracellular potassium. This release rate is negligible for low potassium concentrations. When $\left[\mathrm{K}^{+}\right]_{e}$ exceeds a critical concentration, however, the cell spontaneously generates action potentials (i.e. without synaptic input), which leads to a large efflux of more potassium (Chapter 3),[22]. Movement of this excess of potassium to neighboring tissue along its concentration gradient can generate a SD.

From the simulation shown in figure 4.1, we further observe that the release rate of potassium, $R(C)$, can be approximated with a step function of the extracellular 
potassium concentration $\mathrm{C}$ :

$$
R(C)=R_{0} \cdot H\left(C-C_{t}\right) .
$$

Here $H$ denotes the Heaviside step function $(H(x)=1$ when $x>0$ and 0 otherwise), $C_{t}$ denotes the concentration threshold above which neurons expel $\mathrm{K}^{+}$and $R_{0}$ (in $\mathrm{mM} / \mathrm{s}$ ) is the rate at which $\mathrm{C}$ increases due to this expulsion, which we will refer to as release rate. We will show in section 4.2.6 that approximating the release with a simple step function is not an oversimplification, but yields almost the same results as a sigmoidal function. This is important, because neural tissue contains inhomogeneous populations of neurons, for which a sigmoidal reaction rate is a more realistic approximation than a step function.

Summing the expressions for the homeostasis process and the release process yields the total reaction rate:

$$
\frac{d C}{d t}=R_{t}(C)=\overbrace{R_{0} \cdot H\left(C-C_{t}\right)}^{\text {release }}-\overbrace{G \cdot\left(C-C_{0}\right)}^{\text {homeostasis }} .
$$

The derivation of this reaction rate is generally valid. A pathological high concentration of an excitatory substance, when exceeding a certain threshold, can activate mechanisms that release more of this substance. As an example, figure 4.2 shows the total reaction rate for extracellular potassium. Parameters and their typical values are presented in table 4.1. We remark that the function has a shape similar to the polynomials used in other studies on SD [15, 23], with zeros at resting concentration, at a threshold $C_{t}$ and at some large value above threshold. We can use equation 4.3 to calculate waves resulting from the "generation" and diffusion of an excitatory substance.

Note that equation 4.3 can describe the depolarization of the neuronal membrane voltage too, as this is caused by an increase in concentration of excitatory substance, but it does not describe the recovery. The recovery process, however, does not need to be modeled, because its contribution to the reaction rate is significant only at concentrations far above threshold. It can be shown that its influence on the initiation and the propagation of SD can hence be neglected, except for relatively small propagation speeds, which we will discuss in section 4.4.3.

In short, we argue that SD can be locally mediated through release of an excitatory substance, resulting from exceeding a concentration threshold. Positive feedback leads to net release of more excitatory substance, resulting in depolarization. Equation 4.3 describes the reaction rate of a single excitatory substance using the rate of concentration increase due to release $R_{0}$, a concentration threshold $C_{t}$ and a removal constant $G$. Any effects from other substances or processes, can be expressed in terms of their effect on $R_{0}, C_{t}$ and $G$. As an example, extracellular potassium was used, but the derived expression is valid for any excitatory substance. In the next section, we 




Figure 4.2: The reaction rate, $R_{t}=R(C)-G \cdot\left(C-C_{0}\right)$. Parameters are given in table 4.1. The black dot denotes the physiological resting state. Far above threshold, the derived expressions are expected to be inaccurate, depicted by a dashed curve.

Table 4.1: Parameters and their typical values. Taken or calculated from 3. G was chosen to be in a range that allows SD to be elicited.

\begin{tabular}{ll|cc} 
Parameter & & Typical value & \\
\hline$k$ & Diffusion constant & $2 \cdot 10^{-9}$ & $\mathrm{~m}^{2} / \mathrm{s}$ \\
$R_{0}$ & Release rate & 10 & $\mathrm{mM} / \mathrm{s}$ \\
$C_{t}$ & Threshold concentration & 20 & $\mathrm{mM}$ \\
$C_{0}$ & Resting concentration & 4 & $\mathrm{mM}$ \\
$G$ & Removal rate & 0.1 & $\mathrm{~s}^{-1}$
\end{tabular}


will use the expression for the reaction rate to construct a reaction-diffusion equation that describes the propagation of SD.

\subsubsection{A reaction-diffusion equation for $\mathrm{SD}$}

To calculate the propagation velocity and the onset of a SD wave, a reaction-diffusion equation is constructed that describes the release and subsequent movement of excitatory substance through the tissue. Again, extracellular potassium is used as an example.

In general, a reaction-diffusion equation is written as:

$$
\frac{\partial C}{\partial t}=k \nabla^{2} C+R_{t}(C)
$$

$C$ denotes the concentration of a diffusible substance, $R_{t}$ its net production rate and $k$ its effective diffusion constant. The geometry of the extracellular space slows diffusion [24], while transporters may enhance movement. Furthermore, ions do not simply follow Fick's law of diffusion, because they are charged and therefore their diffusion depends on the co- and contra-diffusing ions, as excellently described in [13]. Therefore, the effective diffusion constant needs to be determined experimentally.

Because the cortex can be considered as a 2-dimensional sheet and the wave front is usually wide compared to the length of the wave, the propagation can be described in one dimension. When considering the initiation of spreading depression from a single point in space, two dimensions are needed and it is convenient to use polar coordinates.

In one dimension, equation 4.4 reads:

$$
\frac{\partial C}{\partial t}=k \frac{\partial^{2} C}{\partial x^{2}}+R_{t}(C)
$$

In the previous section, the total reaction rate was derived, using the expulsion of $\mathrm{K}^{+}$by the neurons, $R(C)$, and its removal from the extracellular space by neurons, glia cells and diffusion to the blood, $G \cdot\left(C-C_{0}\right)$. Substituting the reaction rate from equation 4.3 into equation 4.5 results in our main equation:

$$
\frac{\partial C}{\partial t}=k \frac{\partial^{2} C}{\partial x^{2}}+R_{0} \cdot H\left(C-C_{t}\right)-G \cdot\left(C-C_{0}\right) .
$$

The equation for the two-dimensional case in polar coordinates is similar. Solutions of equation 4.6 now describe the onset and propagation of SD. 


\subsubsection{Calculating a traveling wave solution}

Equation 4.6 is a non-ordinary partial differential equation, which is typically difficult to solve analytically. However, exact expressions can be found for traveling wave solutions, the solutions that describe an SD. Numerical solutions show that these solutions exist, that they maintain their shape while traveling and are stable against small perturbations. For more elaborate, mathematically rigorous, analyses of equations of this type for SD, we refer the reader to the work of e.g. Chapuisat [25] and Dahlem [26].

Because the solution of equation 4.6 is a traveling wave, it can be described as $C(z)=C(x-v t)$, where $v$ is the velocity, with the wave traveling in the positive $\mathrm{x}$ direction, assumed to be on the right. The solution can be translated arbitrarily and we conveniently put $C(z=0)=C_{t}$. Note that $\frac{\partial C}{\partial x}=\frac{\partial C}{\partial z}$.

The solution can be split in two parts: $C_{r}(z)$, the part below threshold on the right, and $C_{l}(z)$, the part above threshold on the left, described by:

$$
\begin{aligned}
& \frac{\partial C_{r}}{\partial t}=k \frac{\partial^{2} C_{r}}{\partial z^{2}}-G \cdot\left(C-C_{0}\right), \\
& \frac{\partial C_{l}}{\partial t}=k \frac{\partial^{2} C_{l}}{\partial z^{2}}+R_{0}-G \cdot\left(C-C_{0}\right),
\end{aligned}
$$

respectively. These are solved with the following constraints: continuity of the solution and its derivative,

$$
\begin{aligned}
C_{r}(0) & =C_{l}(0)=C_{t}, \\
\frac{\partial C_{r}}{\partial z}(0) & =\frac{\partial C_{l}}{\partial z}(0),
\end{aligned}
$$

a resting concentration far away from the wave on the right,

$$
C_{r}(\infty)=C_{0}
$$

and the wave keeping a constant shape,

$$
v=-\frac{\partial C_{r}}{\partial t} / \frac{\partial C_{r}}{\partial z}=-\frac{\partial C_{l}}{\partial t} / \frac{\partial C_{l}}{\partial z} .
$$

The constancy of the wave shape can be used to substitute $\frac{\partial C}{\partial t}$ with $-v \frac{\partial C}{\partial z}$ in equations 4.7 and 4.8:

$$
\begin{aligned}
& -v \frac{\partial C_{r}}{\partial z}=k \frac{\partial^{2} C_{r}}{\partial z^{2}}-G \cdot\left(C-C_{0}\right), \\
& -v \frac{\partial C_{l}}{\partial z}=k \frac{\partial^{2} C_{l}}{\partial z^{2}}+R_{0}-G \cdot\left(C-C_{0}\right) .
\end{aligned}
$$


When $G=0$, it is easy to see that solutions to these equations are:

$$
\begin{aligned}
& C_{r}(z)=C_{0}+\Delta C e^{-\lambda_{2} z}, \\
& C_{l}(z)=C_{t}-\frac{R_{0}}{v} z,
\end{aligned}
$$

with

$$
\begin{aligned}
\Delta C & =C_{t}-C_{0}, \\
\lambda_{2} & =v / k .
\end{aligned}
$$

Continuity of the derivative at $z=0$ can then be used to determine $v$ :

$$
\begin{aligned}
\frac{\partial C_{r}}{\partial z}(0) & =\frac{\partial C_{l}}{\partial z}(0) \\
-\frac{R_{0}}{v} & =-\Delta C \frac{v}{k}
\end{aligned}
$$

From which it follows that

$$
v=\sqrt{\frac{k R_{0}}{\Delta C}} .
$$

When $G \neq 0$, the physically relevant solutions to equations 4.13 and 4.14 can be calculated by using an exponential Ansatz and solving the characteristic polynomial:

$$
\begin{aligned}
& C_{r}(z)=C_{0}+\Delta C e^{\lambda_{-} z}, \\
& C_{l}(z)=C_{0}+\frac{R_{0}}{G}-\left(\frac{R_{0}}{G}-\Delta C\right) e^{\lambda_{+} z},
\end{aligned}
$$

with

$$
\begin{aligned}
& \Delta C=C_{t}-C_{0}, \\
& \lambda_{ \pm}=\frac{-v \pm \sqrt{v^{2}+4 k G}}{2 k} .
\end{aligned}
$$

Again continuity of the derivative is used to determine $v$ :

$$
\begin{aligned}
\frac{\partial C_{r}}{\partial z}(0) & =\frac{\partial C_{l}}{\partial z}(0) \\
\Delta C \lambda_{-} & =-\left(\frac{R_{0}}{G}-\Delta C\right) \lambda_{+} \\
\lambda_{-} & =\lambda_{+}\left(1-\frac{1}{\hat{G}}\right)
\end{aligned}
$$


Table 4.2: Quantities and their scaling.

\begin{tabular}{cccc|c}
\multicolumn{3}{c|}{ Quantity } & Units \\
\hline$x=$ & $\sqrt{k \frac{\Delta C}{R_{0}}}$ & $\times \hat{x}$ & $\mathrm{~m}$ \\
$t$ & $=$ & $\frac{\Delta C}{R_{0}}$ & $\times \hat{t}$ & $\mathrm{~s}$ \\
$C=$ & $C_{0}+(\Delta C$ & $\times \hat{C})$ & $\mathrm{mM}$ \\
$G=$ & $\frac{R_{0}}{\Delta C}$ & $\times \hat{G}$ & $\mathrm{~s}^{-1}$
\end{tabular}

with $\hat{G}=G \frac{\Delta C}{R_{0}}$. This last equation could be solved by writing $\lambda_{-} / \lambda_{+}$in terms of $v$. However, the algebra is slightly simplified by using an identity obtained from the characteristic polynomials:

$$
v=k \lambda_{+}-\frac{G}{\lambda_{+}}=k \lambda_{-}-\frac{G}{\lambda_{-}} .
$$

The last equality can be solved for $\lambda_{-}$by substituting $\lambda_{+}$from equation 4.28 , resulting in:

$$
\lambda_{-}=-\sqrt{1-\hat{G}} \cdot \sqrt{\frac{R_{0}}{k \Delta C}} .
$$

After elementary algebra the velocity $v$ is obtained:

$$
v=\frac{1-2 \hat{G}}{\sqrt{1-\hat{G}}} \cdot \sqrt{\frac{k R_{0}}{\Delta C}} \approx(1-2 \hat{G}) \cdot \sqrt{\frac{k R_{0}}{\Delta C}} .
$$

The last approximation underestimates the solution no more than $0.08 \cdot \sqrt{\frac{k R_{0}}{\Delta C}}$ on the interval $\hat{G}=\left[0, \frac{1}{2}\right]$ and is exact on the endpoints.

\subsubsection{Scaling the equation}

For numerical calculations and dimension analysis, it is convenient to scale $C, x$ and $t$. This yields an equation that depends on only one parameter, $\hat{G}$ :

$$
\frac{\partial \hat{C}}{\partial \hat{t}}=\frac{\partial^{2} \hat{C}}{\partial \hat{x}^{2}}+H(\hat{C}-1)-\hat{G} \hat{C} .
$$

In polar coordinates the equation is scaled similarly. The scaled quantities, denoted with a hat, are listed in table 4.2.

\subsubsection{Calculating the critical stimulus strength}

In various pathological conditions, a large release of potassium or glutamate into extracellular space can elicit a spreading depression. Such a release can for example be 
caused by excessive activity of neurons, or cell membrane damage. Again, we use extracellular potassium as an example. We assess the susceptibility of the modeled tissue to spreading depression by calculating whether or not a local release of potassium elicits a SD. This depends on the total amount of potassium released and on the duration of this stimulus. This is similar to determining the excitability of a neuron with an electrical stimulus. In that case, the current amplitude versus minimum pulse duration can be determined, which is characterized by a rheobase, a minimum charge and chronaxy. The same framework can be applied to SD [20]. We will limit our considerations to either a very short pulse stimulus or continuous stimulation by potassium injection, similar to determining the minimum charge and the rheobase of an electrical stimulus. We did not investigate the effect of the (spatial) width of the stimulus.

In the first scenario, a short release or injection of a certain amount of potassium is modeled with a delta pulse at $t=0$ at the origin. The stimulus results in a very high concentration of substance around the origin, which diffuses away into a larger area. Around the origin, the concentration is above the threshold concentration and therefore potassium is released by the neurons. An SD is generated only when more extracellular potassium is generated than diffuses away. Provided $\hat{G}<1 / 2$, this is the case when the amount of injected potassium is large enough.

In the second scenario, a stimulus of infinite duration is modeled as a point source of potassium at the origin. The steady flux of potassium leads to a build-up of concentration around the source. If the stimulus is weak, an equilibrium is reached between the generation of potassium and its diffusion and removal. If the source is stronger, such an equilibrium does not exist and a traveling wave of high potassium is induced.

Calculating the initiation of SD in one dimension underestimates the effectiveness of diffusion and therefore polar coordinates need to be used. We use the scaled equation, equation 4.32 , in polar coordinates. This allows us to calculate numerical results for different $\hat{G}$ and scale the results depending on $k, \Delta C$ and $R_{0}$, rather than simulating the full 4-parameter space. The equation reads:

$$
\frac{\partial \hat{C}}{\partial \hat{t}}=\frac{1}{\hat{r}} \frac{\partial \hat{C}}{\partial \hat{r}}+\frac{\partial^{2} \hat{C}}{\partial \hat{r}^{2}}+H(\hat{C}-1)-\hat{C} \hat{G}+\hat{P} \delta(\hat{t}, \hat{x}, \hat{y})+\hat{S} \delta(\hat{x}, \hat{y}) .
$$

Here

$$
P=\frac{k \Delta C^{2}}{R_{0}} \hat{P}
$$

is the amount of excitatory substance injected at $\mathrm{t}=0$ at the origin (in $\mathrm{mM} \cdot \mathrm{m}^{2}$ ) and

$$
S=k \Delta C \hat{S}
$$

is the flux of the point source of substance at the origin (in $\mathrm{mM}^{*} \mathrm{~m}^{2} / \mathrm{s}$ ). ${ }^{\dagger}$ The scal-

\footnotetext{
${ }^{\dagger}$ To avoid confusion, we point out that $\mathrm{mM}$ denotes milliMolar $=\mathrm{mmol} / \mathrm{liter}\left(=\mathrm{mol} / \mathrm{m}^{3}\right)$ and hence the use of "mM/liter", as sometimes encountered in literature on SD, is incorrect.
} 
ing of these parameters can be inferred from their units. To obtain the amount of substance that would need to be injected in an experiment, this needs to be multiplied with the thickness of the cortex in which the concentration is increased. We further remark that for a stimulus to be considered short, its duration should be much shorter than both $\Delta C / R_{0}$ and $1 / G$, typically $\ll 1 \mathrm{~s}$. Furthermore, for a stimulus to be considered a point source, it should have a width smaller than $k / v$, typically $<30$ $\mu \mathrm{m}$. This is not the case for most experimental stimuli. However, wider stimuli are expected to behave qualitatively similar to point sources, with the diffusion constant progressively losing influence with increasing stimulus width. Further calculations are needed to investigate the effect of stimuli with finite areas, which was not further explored in this study.

No analytical solution was found for equation 4.33 , neither for $P \neq 0$ nor for $S \neq 0$. Therefore, a numerical solution was obtained, by using a simple finite elements calculation.

The elements were rings with inner and outer radii of $r_{n}$ and $r_{n}+\Delta r$, with $r_{n}=$ $n * \Delta r$ and $n$ ranging from 0 to $L / \Delta r$, where $\mathrm{L}$ is the radius of the simulation area. The areas $A_{n}$ and outer circumferences $O_{n}$ were calculated and the flux between neighboring elements was calculated as $(C[n]-C[n+1])) O_{n} / \Delta x$. The outermost element was kept at $C=C_{0}$, to create an absorbing boundary condition, although the simulation area was large enough to not let the solution reach the boundary. The changes in concentration were then calculated with an explicit time stepping scheme.

$\Delta x$ was decreased, until no significant changes in simulation results were observed. The time step was chosen as $0.05 / \Delta x^{2}$ to ensure convergence. Typical values used were $L=30, \Delta x=0.1$ and simulation duration $T=10$. For various values of $\hat{G}$, the smallest values for both S and P that were able to induce SD were then obtained empirically.

\subsubsection{Using a sigmoid instead of a step function}

For tissue containing many neurons with inhomogeneous properties and varying inputs, a sigmoid is a better approximation to the release rate of potassium then a step function. To test whether this yields different results, the numerical calculations are done with a sigmoid instead of a step function. A sigmoid as wide as possible without having a significant slope at the origin was used. Figure 4.3(left) shows this sigmoid together with the original step function. Figure 4.3(right) compares the two waveforms. Even for this width of the sigmoid, the differences in shape and velocity are very small compared to the usually encountered variations in experimental measurements. Furthermore, numerical solutions show that the critical steady stimulus $f_{S}$ is smaller when $R(C)$ is a sigmoid instead of a step function and the critical short stimulus $f_{P}$ is slightly larger, but the behavior of the functions is similar.

From this we conclude that a step function yields practically the same results as 

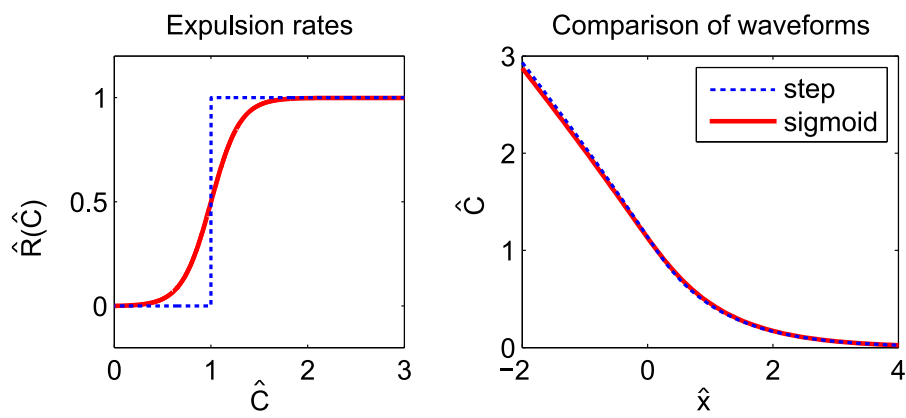

Figure 4.3: Comparison of SD waveforms for different expulsion functions. A sigmoid, $R(\hat{C})=1 /(1+\exp (-(\hat{C}-1) / 0.15))-1 /(1+\exp (1 / 0.15))$, is compared with the step function used throughout this chapter (left graph). The right graph shows how similar the two waveforms are. The propagation velocity when using a sigmoid is only $2 \%$ faster. The waveforms were calculated numerically from equation 4.32, with $\hat{G}=0.07$.

a sigmoidal function when calculating the propagation and initiation of SD.

\subsubsection{Comparison with experimental measurements from literature}

As an initial validation of the model, its solution will be compared with the time courses of the extracellular potassium concentration measured during experimentally induced SDs. Two concentration time-courses were obtained from literature. These measurements were chosen for their good time resolution, allowing us to obtain several data points during the onset of SD. The graphs were digitized manually from the figures using Matlab.

A first set of data was taken from figure 1A of [27], who elicited SD in rats of both sexes in the frontoparietal cortex. A filter paper soaked with $1 \mathrm{M} \mathrm{KCl}$, applied from an opening in the skull, induced the SD. The potassium waveform was measured 5 $\mathrm{mm}$ away from the stimulus location. A second experimental result was obtained from figure 2 of [7], where SD was elicited in a female rat in the dorsal hippocampus by pressure injection of potassium. A propagation speed was obtained in both papers by measuring the arrival times of the SD wave at two electrodes. The values at the center of the observed velocity ranges were used. These were 60 and $100 \mu \mathrm{m} / \mathrm{s}$, respectively.

For each data set, the interval was selected in which the concentration first increases exponentially and then rises linearly. The solution to equation 4.6 was fitted to the data points in this interval by a least-squares method with $\hat{G}, C_{0}, \Delta C$ and $R_{0}$, independent of $k$, which was set to yield the correct propagation speed. 


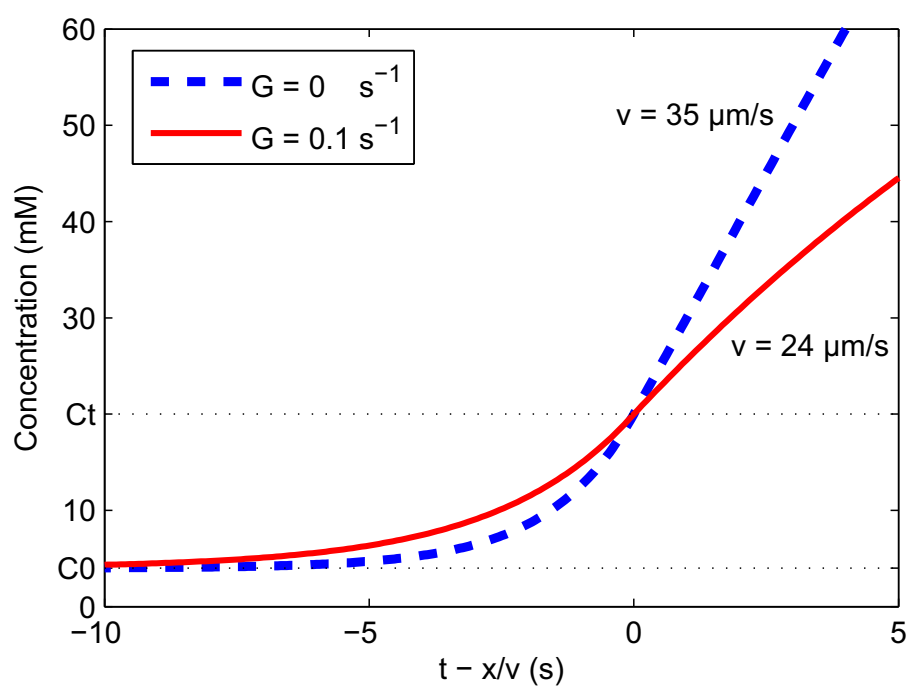

Figure 4.4: Traveling wave solutions of equation 4.6. Shown are results both for no removal, $G=0$ (dashed line) and a fairly large removal rate of $G=0.2 / s$ (solid line). Parameters are given in table 4.1. The propagation velocity of the waveforms was calculated as 35 and 24 $\mu \mathrm{m} / \mathrm{s}$, respectively.

\subsection{Results}

In the previous section, we derived a reaction diffusion-equation and calculated the concentration time courses and propagation velocity of SD as caused by an excitatory diffusible substance, such as potassium or glutamate. Furthermore, we assessed tissue susceptibility to SD, by calculating the minimum amplitude of a stimulus that triggers SD.

\subsubsection{Concentration time courses and propagation speed}

We calculated a traveling wave solution to equation 4.6, which describes an SD. Two examples of this solution with numerical values for extracellular potassium are shown in figure 4.4. The concentration time course during SD is characterized by an exponential increase that lasts until the concentration reaches the threshold $C_{t}$, followed by an almost linear rise.

When glial buffering is negligible, i.e. $G=0$, the corresponding speed of the wave is:

$$
v_{0}=\sqrt{\frac{k R_{0}}{\Delta C}} .
$$

This equation shows that when the removal rate of potassium is negligible, e.g. after complete ischemia, the propagation speed increases as the square root of the effective 
diffusion constant $k$ (as holds for any chemical wave [28]). The speed also increases as the rate of concentration rise $R_{0}$ due to expulsion of potassium increases, for example when the extracellular space is smaller. The speed of the SD wave also increases when the difference between resting and threshold concentration $\Delta C$ is decreased, for example by hyperkalemia or a concurrent rise of glutamate, which lowers the threshold concentration.

Considering the scenario where there is removal of extracellular $K^{+}$, for example by glia cells, bloodstream and $\mathrm{Na} / \mathrm{K}$-pumps, the conditions that result in the generation in a SD are slightly different. We find that a larger stimulus is needed to induce SD and SD will propagate slower. A useful quantity to calculate this is the normalized removal constant $\hat{G}=G \frac{\Delta C}{R_{0}}$. In the methods section it is derived that the propagation velocity is approximately multiplied with a factor $1-2 \hat{G}$ :

$$
v_{1} \approx(1-2 \hat{G}) \cdot v_{0}=(1-2 \hat{G}) \cdot \sqrt{\frac{k R_{0}}{\Delta C}} .
$$

Note that this implies that for fast normalized removal rates $\hat{G}>1 / 2$, the depolarization cannot propagate at all. Intuitively, one may suspect that the depolarization cannot propagate when diffusion is very slow either. This case, in which recovery cannot be neglected, is discussed in section 4.4.4.

\subsubsection{Critical stimulus for triggering spreading depression}

To obtain a measure for the susceptibility of tissue to $\mathrm{SD}$, we calculated both the minimum amount of excitatory substance in a short pulse $P$ and the minimum flux delivered by continuous stimulation $S$, that is sufficient to elicit SD. The critical amplitudes $P$ and $S$ are functions of $\hat{G}$ that scale with $k, \Delta C$ and $R_{0}$ and are given by

$$
P_{c r i t}=\frac{k \Delta C^{2}}{R_{0}} \cdot f_{P}\left(\frac{G \Delta C}{R_{0}}\right)
$$

and

$$
S_{c r i t}=k \Delta C \cdot f_{S}\left(\frac{G \Delta C}{R_{0}}\right),
$$

respectively. Here $f_{p}(\hat{G})$ and $f_{S}(\hat{G})$, shown in figure 4.5 , are functions that depend only on the normalized removal rate and were determined numerically. It can be observed for both stimulus types that SD is more difficult to induce in tissue that has a higher diffusion constant, larger concentration threshold $\Delta C$, a smaller release rate $R_{0}$ and a faster removal constant $G$. 

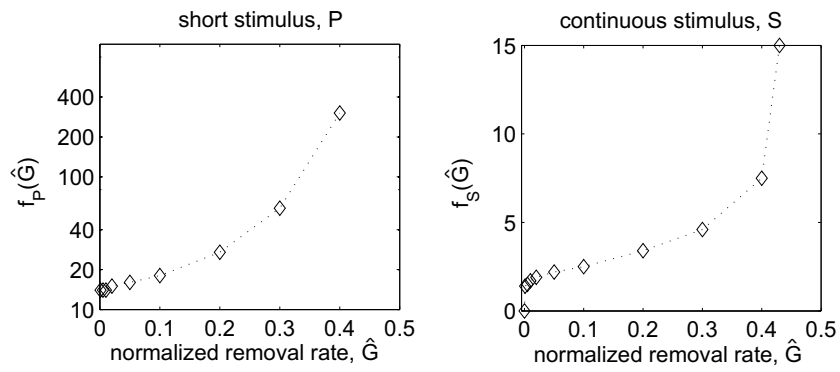

Figure 4.5: The strengths of the critical stimuli, calculated as a function of $\hat{G}$ as described in section 4.2.5. The line drawn serves as a guide for the eye.

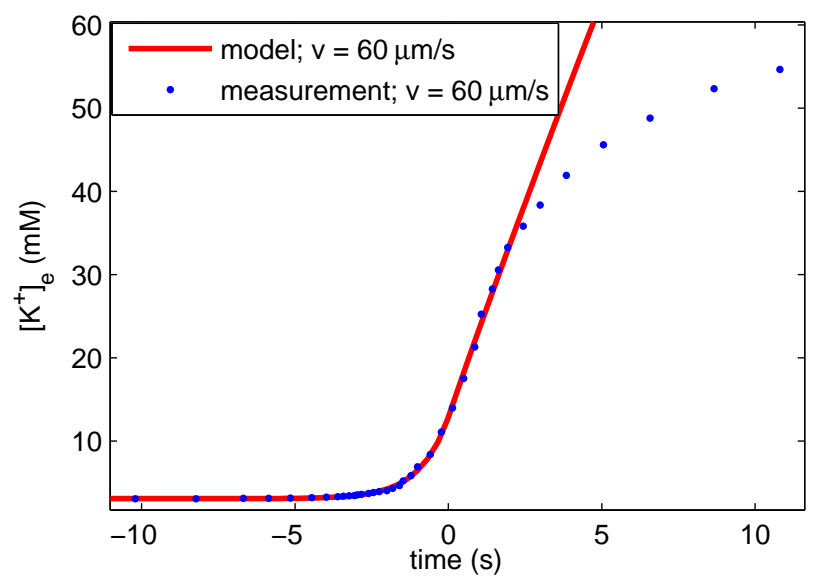

Figure 4.6: Comparison of the model's solution with experimental data, taken from figure 1A of [27]. The calculated and observed velocities of the waves are indicated in the legend. Dots are points digitally read out from the original graph. 


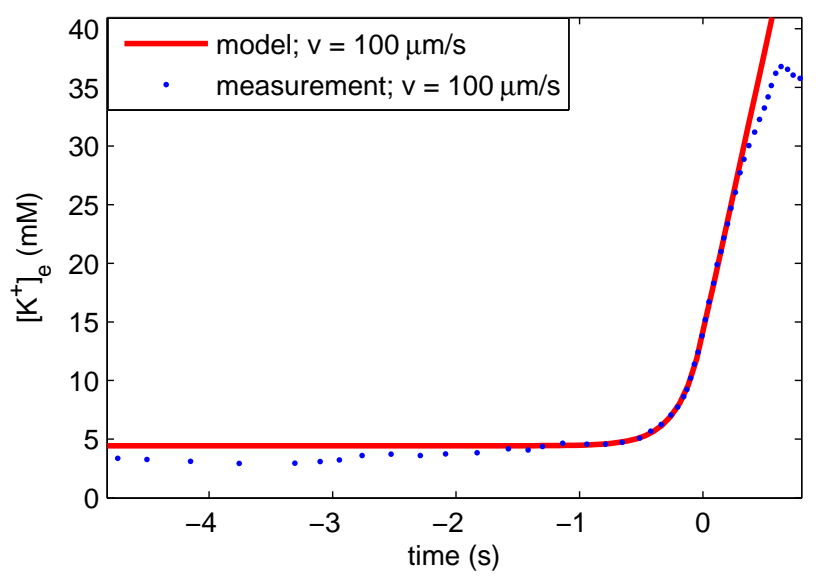

Figure 4.7: Comparison of the model's solution with data, taken from figure 2 of [7]. The calculated and observed velocities of the waves are indicated in the legend. Dots are points digitally read out from the original graph.

\subsubsection{Comparison with experimental time courses}

Figure 4.6 compares a modeled time course with the measurement obtained from [27]. The obtained parameter values are: $C_{0}=3.1 \mathrm{mM}, R_{0}=11 \mathrm{mM} / \mathrm{s}, \Delta C=9.4 \mathrm{mM}$, $k=3.4 \cdot 10^{-9} \mathrm{~m}^{2} / \mathrm{s}, G=0.02 / \mathrm{s}$. These values are in the physiological range and the model correctly describes the shape of the measured time trace: an exponential rise followed by a steep almost linear rise.

Figure 4.7 compares a modeled time course with a second measurement, from [7]. Here we find: $C_{0}=4.4 \mathrm{mM}, R_{0}=48 \mathrm{mM} / \mathrm{s}, \Delta C=9.0 \mathrm{mM}, k=1.9 \cdot 10^{-9} \mathrm{~m}^{2} / \mathrm{s}, G$ $=0.00 / \mathrm{s}$. These values are also in the physiological range.

Note that only the velocity ranges for the individual measured time courses are known with a typical spread of $20 \%$, which leads to large errors, $\pm 40 \%$, in the determined diffusion constants.

\subsubsection{Predictions of the model}

\section{Square root dependence of propagation speed on diffusion constant}

Our model predicts a square root dependence of the propagation velocity on the effective diffusion constant $k$ (equation 4.37).

This is in accordance with the findings of Shapiro [13], who calculates with a detailed numerical model the propagation velocity of SD as a function of $k$ for potassium. In his model, potassium diffuses mainly intercellularly through gap junctions, rather than extracellularly. He finds that the propagation speed of SD increases as $\sqrt{k}$. 
The velocity deviates from the square root dependence for small values of $k$, where it drops rapidly to zero and SD cannot be elicited below a certain value of $k$. We will discuss later in more detail that for very slow diffusion, the limits on the total amount of substance that can be released cannot be neglected. This lowers the propagation speed and prohibits SD for very small diffusion constants.

\section{SD cannot be induced when the concentration increase due to release is small}

According to equation 4.37, no SD can be elicited when $G>R_{0} /(2 \Delta C)$. Small rates of concentration rises, $R_{0}$, therefore prevent $\mathrm{SD}$. It is indeed observed that $\mathrm{SD}$ cannot be elicited in brains of young animals, in which the extracellular space is relatively large. Expelled potassium and neurotransmitters are diluted more, and cause a smaller rise in extracellular concentrations, thereby protecting against SD [29].

\section{Tissue susceptibility to SD and its propagation speed are not necessarily corre- lated}

Equations 4.37 - 4.39 predict that either decreasing $R_{0}$ or increasing $\Delta C$ or $G$ both lowers the propagation speed and increases the critical stimulus amplitude for triggering SD. Decreasing the effective diffusion constant $k$ also lowers the propagation speed, but decreases the critical stimulus amplitude. Hence, the intuitive idea that SD is more difficult to induce in tissue where it propagates slower, is incorrect in cases where the effective diffusion constant changes. This lack of correlation was indeed observed by Weimer et al. [30]. Their experiments in chick retina show that during the recovery period that follows an $\mathrm{SD}$, the threshold for electrical stimulation is increased, while cooling the tissue decreases this threshold. Both manipulations slow the propagation of SD.

Our model predicts from this measurement that cooling decreases the effective diffusion constant. It also quantifies this. Weimer et al. cooled the tissue from 30 to $20{ }^{\circ} \mathrm{C}$. This resulted in a reduction of propagation speed of $30 \%$, in combination with a decreased threshold for electrical stimulation of 50\%. These values both correspond with a decrease in diffusion constant of $50 \%$ (equation 4.37 and 4.38. In comparison, this cooling slows (passive) diffusion of potassium in water with only $20 \%$ [31]. Hence, our model predicts that passive diffusion of extracellular potassium cannot have a primary role in the propagation of SD in these experiments. Either potassium is actively transported, for example by the glia, or SD propagates through another mechanism such as axonal conduction of action potentials or diffusion of glutamate.

We have to be careful in drawing conclusions from the electrical stimulation threshold alone, since our model describes the stimulus amplitude in terms of substance release, which may not be proportional to the injected current and also may change upon cooling. 
Threshold concentration for SD is lower in female rat, while propagation speed is the same

Our model predicts that a lower concentration threshold increases the propagation speed (equation 4.37). This is in disagreement with observations from Adamek [27], who elicited SD by applying a cotton ball with $1 \mathrm{M} \mathrm{KCl}$ to the frontoparietal cortex. They measured the time course of the extracellular potassium concentration during the onset of SD close $(1.5 \mathrm{~mm})$ to the stimulus site. A measure for the concentration threshold was obtained by plotting this time course on a semi-logarithmic scale, which shows a deflection point. This point was found at much lower concentrations in female rat cortex $(5.0 \pm 0.6 \mathrm{mM})$ than in male rat cortex $(11.4 \pm 0.4 \mathrm{mM})$ above the resting concentration $(3.0 \mathrm{mM})$. The corresponding propagation speed for $\mathrm{SD}$, was determined to be $3.9 \pm 0.3 \mathrm{~mm} / \mathrm{min}$ in females and $3.6 \pm 0.3 \mathrm{~mm} / \mathrm{min}$ in males. The difference is not statistically significant.

Our simulations show that this deflection point in the semi-logarithmic plot is not a reliable measure of the concentration threshold, because it is influenced by the SD wave that is generated at the stimulus site. However, their measurements definitely suggest a concentration threshold that is lower in female rat, although maybe less pronounced than the deflection points suggest.

As the lower concentration threshold for triggering does not have a large effect on the propagation speed, we predict that diffusing potassium has only a secondary role in the propagation of normoxic SD. The hypothesis that a lower concentration threshold for potassium leads to a higher susceptibility to SD is in agreement with the predictions of our model (equation 4.38 and 4.39).

\subsection{Discussion}

The main results of this work are the analytical expressions for the dynamics of diffusible excitatory substances during SD and how these contribute to the triggering and propagation. Expressions were derived for the propagation speed (equations 4.36 and 4.37) and the minimum amplitude of a stimulus that is sufficient to induce SD (equations 4.38 and 4.39). The theoretical concentration time courses during the onset of SD were compared with experimental recordings of extracellular potassium (figures 4.6 and 4.7 ) and several predictions were made with the model by analyzing observations from literature.

\subsubsection{Role of neural excitability}

The most important assumption that we make to arrive at the expressions for the propagation and triggering, equations 4.36-4.39, is that the release rate of a substance is a function of its own concentration that can be approximated with a step function or 
sigmoid. Although our results do not depend on the exact mechanism, we hypothesized that neuronal excitation mediates such a release rate of potassium and/or glutamate. However, it has been found that SD can still occur when TTX blocks sodium channels [32,33], showing that the generation of action potentials is not necessary for SD. In this case, depolarization of the neuronal membrane can still mediate SD. Also with TTX applied, a critical value for the neuronal resting membrane voltage is observed above which SD is triggered. This is a few $\mathrm{mV}$ higher than when TTX is not applied [33]. Hence, depolarization of the neuronal cell membrane by excitatory effects could mediate a pathological release of excitatory substances. This hypothesis can be tested by slowly increasing the glutamate or the potassium concentration in a large area and verifying that the threshold resting membrane voltages above which the depolarization is started are similar for both substances.

\subsubsection{Validity of the derived expressions}

The solution to equation 4.6 was compared with two experimental time courses of extracellular potassium (figures 4.6 and 4.7). For both measurements, the theoretical solution describes the measured data very well, until the concentration has risen to approximately two thirds of the maximum concentration reached during the SD. Hereafter, recovery processes, which were not modeled, start to influence the concentration time course. In both cases, the obtained parameter values are in the physiological range.

Furthermore, the solution also deviates during the earliest phase of the SD wave from the data set of Herreras et al. [7],(figure 4.7). Rather than an exponential increase in concentration, the concentration initially rises approximately linearly. The start of this rise $(t=-3.5 \mathrm{~s})$ occurs simultaneously with the onset of prodromal spiking activity that Herreras et al. observed in extracellular recordings. This activity can cause the initially linear rise in $\left[K^{+}\right]_{e}$. Note that we only described the contribution of diffusing substances on the propagation of $\mathrm{SD}$, and not the possible contributions of other mechanisms, notably the propagation of neural activity through synaptic connections. This has been investigated, although not applied to SD, for constant ion concentrations [34], as well as numerically, for dynamic ion concentrations [35].

Also, Herreras et al. observed a large change in extracellular potential, associated with neuronal depolarization, before the fast rise of $\left[K^{+}\right]_{e}$. Therefore, extracellular potassium seems to be a byproduct, rather than the primary contribution to the propagation of SD in this experiment. Still, a critical point can be observed, after which a fast linear rise in potassium concentration occurs, preceded by an exponential increase, as caused by diffusion.

Although the model describes the experimental time courses of the concentration of extracellular potassium very well, this does not mean that diffusing extracellular potassium is the primary mechanism for SD propagation. For the release of a sec- 
ondary substance, the same reaction-diffusion equation is valid. In that case, however, a different value for the concentration threshold is observed. We will discuss this in the next section.

\subsubsection{Multiple diffusing substances}

The release rate of one excitatory substance, e.g. potassium, can be influenced by the concentration of a second substance, e.g. glutamate. A rise in extracellular glutamate concentration lowers the concentration of potassium at which a certain membrane voltage is reached. If the release mechanism is mediated by the membrane voltage, this effectively lowers the potassium concentration threshold for potassium release. This results in a higher propagation speed (equation 4.37).

Two threshold concentrations can then be distinguished for a single substance. The threshold for (experimentally) inducing SD, when no other substance is released yet, and the observed threshold during propagation. The latter is lower due to the influence of a second excitatory substance. How close the threshold during propagation is to the threshold for inducing SD is a measure for the relative importance of a substance in the propagation of SD.

The model can be extended by adding an equation for a second substance to it and describing the concentration threshold of both substances as a function of concentration of the other.

\subsubsection{Effects of recovery and limited total amount of released substance}

In deriving equation 4.6, it is assumed that cells in the tissue, once the concentration rises above the threshold, expel excitatory substance indefinitely. This assumption is justified in most cases, because only the front of the wave is important for an accurate description of propagation and initiation. Even for a diffusion constant close to zero, the model predicts that propagation of SD is possible, albeit slow. In that case, the concentration at a certain position in the tissue keeps rising, such that the concentration gradient eventually is steep enough to cause sufficient flux to the interstitial space of the neighboring tissue. This cannot occur in reality, because there is a natural limit on the concentration and the total amount of substance that can be released from the cells.

Therefore, when equation 4.37 predicts relatively slow propagation velocities, these are either overestimated or propagation is not possible. The propagation velocity is considered slow, when the front of the waveform is affected by recovery effects.

To investigate this, recovery effects can be incorporated in the numerical model. This would also allow for investigating how consecutive SD's are induced by a prolonged stimulus. These effects can be incorporated, for example, by introducing a 
recovery variable in the model [23]. Such a variable slowly increases when the concentration is high and limits the release rate and enhances removal.

\subsection{Conclusion}

Concentration dynamics of diffusible excitatory substances, such as potassium and glutamate, were calculated during triggering and propagation of spreading depression (SD). For this purpose, a reaction-diffusion equation was constructed from four physiological parameters. Solutions to the equation describe the onset of SD and yield an analytical solution of the shape of the front of the wave, e.g. concentration as a function of position and time, and the propagation speed (equation 4.36 and 4.37). A critical stimulus strength was calculated for both prolonged and short stimuli (equations 4.38 and 4.39).

The solution to our equations was validated with experimental measurements of extracellular potassium concentration and was found to describe the onset of the concentration time course very well. The estimates for the parameters are all in the physiological range. Predictions of the model were presented and it was shown how the obtained expressions can be used to analyze several experimental findings. The model can be elaborated to describe multiple substances or recovery effects.

The presented expressions allow an analysis of the influence of an excitatory substance on SD propagation and triggering, in terms of concentration threshold, release rate, removal rate and effective diffusion constant. The effects of other influences can be described by how they change these quantities. By comparing them with experiments or more elaborate numerical models, the derived expressions may be helpful in answering the question of which role diffusible substances play in various forms of SD.

\section{References}

[1] A. A. P. Leao, "Further observations on the spreading depression of activity in the cerebral cortex.", J Neurophysiol 10, 409-414 (1947).

[2] H. Nakamura, A. J. Strong, C. Dohmen, O. W. Sakowitz, S. Vollmar, M. Su, L. Kracht, P. Hashemi, R. Bhatia, T. Yoshimine, J. P. Dreier, A. K. Dunn, and R. Graf, "Spreading depolarizations cycle around and enlarge focal ischaemic brain lesions.”, Brain 133, 1994-2006 (2010).

[3] J. P. Dreier, "The role of spreading depression, spreading depolarization and spreading ischemia in neurological disease.”, Nat Med 17, 439-447 (2011).

[4] G. G. Somjen, "Mechanisms of spreading depression and hypoxic spreading depression-like depolarization.”, Physiol Rev 81, 1065-1096 (2001). 
[5] M. Lauritzen, J. P. Dreier, M. Fabricius, J. A. Hartings, R. Graf, and A. J. Strong, "Clinical relevance of cortical spreading depression in neurological disorders: migraine, malignant stroke, subarachnoid and intracranial hemorrhage, and traumatic brain injury.", J Cereb Blood Flow Metab 31, 17-35 (2011).

[6] B. Grafstein, "Subverting the hegemony of the synapse: complicity of neurons, astrocytes, and vasculature in spreading depression and pathology of the cerebral cortex.", Brain Res Rev 66, 123-132 (2011).

[7] O. Herreras and G. G. Somjen, "Propagation of spreading depression among dendrites and somata of the same cell population.”, Brain Res 610, 276-282 (1993).

[8] C. Largo, G. C. Tombaugh, P. G. Aitken, O. Herreras, and G. G. Somjen, "Heptanol but not fluoroacetate prevents the propagation of spreading depression in rat hippocampal slices.", J Neurophysiol 77, 9-16 (1997).

[9] O. Herreras, "Electrical prodromals of spreading depression void grafstein's potassium hypothesis.", J Neurophysiol 94, 3656; author reply 3656-3656; author reply 3657 (2005).

[10] M. Ghergu, Nonlinear PDEs mathematical models in biology, chemistry and population genetics (Springer-Verlag Berlin Heidelberg, Heidelberg New York) (2012).

[11] H. C. Tuckwell and R. M. Miura, “A mathematical model for spreading cortical depression.”, Biophys J 23, 257-276 (1978).

[12] H. C. Tuckwell, "Simplified reaction-diffusion equations for potassium and calcium ion concentrations during spreading cortical depression.”, Int J Neurosci 12, 95-107 (1981).

[13] B. E. Shapiro, "Osmotic forces and gap junctions in spreading depression: a computational model.", J Comput Neurosci 10, 99-120 (2001).

[14] W. Yao, H. Huang, and R. M. Miura, "A continuum neuronal model for the instigation and propagation of cortical spreading depression.”, Bull Math Biol 73, 2773-2790 (2011).

[15] B. Grafstein, Neuronal release of potassium during spreading depression., chapter Brain Function, Vol. I. (University of California Press, Berkeley, Calif. 87.) (1963).

[16] J. Bureš, O. Buresová, and J. Křivánek, The mechanism and applications of Leão's spreading depression of electroencephalographic activity (Academia, t. [ST 5]) (1974).

[17] H. Wilson, Spikes, Decisions, and Actions: The Dynamical Foundations of Neuroscience (Oxford University Press) (1999).

[18] F. Schlögl, "Chemical reaction models for non-equilibrium phase transitions", Zeitschrift für Physik 253, 147-161 (1972).

[19] C. F. Starmer, "Initiation of excitation waves", Scholarpedia 2, 1848 (2007).

[20] I. Idris and V. N. Biktashev, "Analytical approach to initiation of propagating fronts.", Phys Rev Lett 101, 244101 (2008).

[21] Y. B. Zeldovich and D. A. Frank-Kamenetskii, "Theory of thermal flame propagation", Zh. Fiz. Khim. 12, No. 1, 100105 (1938). 
[22] H. Kager, W. J. Wadman, and G. G. Somjen, "Conditions for the triggering of spreading depression studied with computer simulations.”, J Neurophysiol 88, 2700-2712 (2002).

[23] J. A. Reggia and D. Montgomery, "Modeling cortical spreading depression., Proc Annu Symp Comput Appl Med Care 873-877 (1994).

[24] E. Syková and C. Nicholson, "Diffusion in brain extracellular space.”, Physiol Rev 88, 1277-1340 (2008).

[25] G. Chapuisat and R. Joly, "Asymptotic profiles for a traveling front solution of a biological equation", Mathematical models \& methods in applied sciences 21, 2155-2177 (2011).

[26] M. A. Dahlem, R. Graf, A. J. Strong, J. P. Dreier, Y. A. Dahlem, M. Sieber, W. Hanke, K. Podoll, and E. Schll, "Two-dimensional wave patterns of spreading depolarization: Retracting, re-entrant, and stationary waves”, Physica D: Nonlinear Phenomena 239, 889 - 903 (2010).

[27] S. Adámek and F. Vyskočil, "Potassium-selective microelectrode revealed difference in threshold potassium concentration for cortical spreading depression in female and male rat brain.", Brain Res 1370, 215-219 (2011).

[28] K. Showalter and J. Tyson, "Luther's 1906 discovery and analysis of chemical waves", Journal of Chemical Education 64, 742-744 (1987).

[29] G. G. Somjen, Ions in the Brain - Normal Function, Seizures, and Stroke, chapter 18 (Oxford University Press) (2004).

[30] M. S. Weimer and W. Hanke, "Propagation velocity and triggering threshold of retinal spreading depression are not correlated.”, Exp Brain Res 164, 185-193 (2005).

[31] T. Bastug and S. Kuyucak, "Temperature dependence of the transport coefficients of ions from molecular dynamics simulations", Chemical Physics Letters 408, 84 - 88 (2005).

[32] C. Tobiasz and C. Nicholson, "Tetrodotoxin resistant propagation and extracellular sodium changes during spreading depression in rat cerebellum.”, Brain Res 241, 329-333 (1982).

[33] M. Müller and G. G. Somjen, " $\mathrm{Na}(+)$ and $\mathrm{k}(+)$ concentrations, extra- and intracellular voltages, and the effect of ttx in hypoxic rat hippocampal slices.", J Neurophysiol 83, 735-745 (2000).

[34] S. Amari, "Dynamics of pattern formation in lateral-inhibition type neural fields.", Biol Cybern 27, 77-87 (1977).

[35] G. Ullah, J. R. Cressman, Jr, E. Barreto, and S. J. Schiff, "The influence of sodium and potassium dynamics on excitability, seizures, and the stability of persistent states. ii. network and glial dynamics.", J Comput Neurosci 26, 171-183 (2009). 


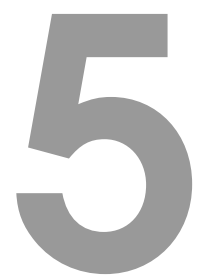

\title{
Single neuron dynamics during experimentally induced anoxic depolarization *
}

\begin{abstract}
We studied single neuron dynamics during anoxic depolarizations, which are often observed in cases of neuronal energy depletion. Anoxic and similar depolarizations play an important role in several pathologies, notably stroke, migraine, and epilepsy. One of the effects of energy depletion was experimentally simulated in slices of rat cortex by blocking the sodium-potassium pumps with ouabain. The membrane voltage of pyramidal cells was measured. Five different kinds of dynamical behavior of the membrane voltage were observed during the resulting depolarizations. Using bifurcation analysis of a single cell model, we show that these voltage dynamics all are responses of the same cell, with normally functioning ion channels, to particular courses of the intra- and extracellular concentrations of sodium and potassium.
\end{abstract}

*Published as: BJ Zandt, T Stigen, B ten Haken, T Netoff, MJAM van Putten (2013) Single neuron dynamics during experimentally induced anoxic depolarization. Journal of Neurophysiology, 110:14691475 


\subsection{Introduction}

Physiological functioning of neurons is critically dependent on the ion concentration gradients across the cell membranes. Molecular pumps and transporters, notably the sodium-potassium pump, in the membranes of neurons and glia cells work to maintain the physiological intra- and extracellular concentrations of ions and neurotransmitters.

These pumps require a constant supply of energy in the form of ATP. After an ischemic stroke, or during anoxia, the supply of glucose and oxygen from the blood is insufficient for the tissue's metabolic needs. This leads to failure of ion homeostasis. A characteristic phenomenon resulting from this failure is anoxic depolarization, an initial slow increase of the membrane voltage $\left(V_{m}\right)$, followed by a sudden, large depolarization, accompanied by a loss of membrane resistance and large shifts in the intra- and extracellular ion concentrations [1]. Modeling studies [2-5] show that during anoxia/ischemia potassium ions leak into the extracellular space, which causes a slow, gradual increase of $V_{m}$. When the extracellular potassium concentration reaches a certain threshold, the neurons start spiking, causing a large efflux of potassium, resulting in a fast depolarization.

Also, during epileptic seizures, the intense neuronal activity can cause the extracellular potassium concentration to rise out of its physiological range. This initially excites neurons, but may subsequently result in depolarization and cessation of electrical activity. This is a possible mechanism for spontaneous termination of seizures $[3,4,6-8]$.

A phenomenon similar to anoxic depolarization, spreading depression, is the physiological substrate of a migraine aura. Spreading depression is a wave that slowly propagates through the neural tissue, during which there is a large shift in intra- and extracellular ion concentrations, glutamate is released, cells depolarize and electrical activity is depressed $[9,10]$. When blood flow is sufficient, these depolarizations are relatively harmless and cells recover within minutes [11]. However, when blood flow is restricted, as during ischemia, these depolarizations induce massive cell death [12] and are therefore a potential target for therapy.

Despite the importance of anoxic depolarizations in several neurological disorders, the underlying physiology is not yet fully known [13]. This is probably due to the complex interplay of neuronal membrane dynamics, synaptic activity, glial and ion-pump activity, the composition of the extracellular space and blood flow during these depolarizations. Although the membrane voltage dynamics have a central role in the initiation of the rapid depolarization, there is little experimental literature available on these dynamics during anoxic depolarization. Here we present such measurements. We experimentally simulated one of the effects of ischemia by applying ouabain [14-16] to slices of rat brain. This blocks the sodium-potassium pump activity and induces anoxic depolarizations. 
First we will discuss the rich variety of membrane voltage dynamics that was observed during this halt of the $\mathrm{Na} / \mathrm{K}$-pumps. Then we analyze these dynamics in terms of a dynamical system and compare them with the behavior predicted by single neuron models with dynamic ion concentrations $[2,7,17]$.

\subsection{Materials and methods}

\subsubsection{Experimental procedure}

Long Evans rats aged 14-21 days were anesthetized with isoflurane, decapitated and the brain removed. The brain was then blocked to prepare transverse slices of entorhinal cortex and ventral hippocampus. Using a microtome, $400 \mu \mathrm{m}$ thick slices were prepared. The slices were then placed in a submersion chamber containing artificial cerebrospinal fluid (in mM: $125 \mathrm{NaCl}, 25 \mathrm{NaHCO}$, 11 D-glucose, $3 \mathrm{KCl}$, $1.25 \mathrm{NaH} 2 \mathrm{PO} 4,2 \mathrm{CaCl} 2,1 \mathrm{MgCl} 2$ ) and allowed to recover for 1 hour at $37^{\circ} \mathrm{C}$. Brain slices were then transferred to a submerged recording chamber. Neurons were visualized using differential interference contrast (DIC) optics. An extracellular electrode, $\mathrm{Ag} / \mathrm{AgCl}$ microelectrode inside a borosilicate micropipette, was placed in layer $2 / 3$ of entorhinal cortex. Based on its morphology, a pyramidal cell was selected for patching, located approximately $40 \mu \mathrm{m}$ adjacent to the extracellular electrode. Intracellular recording electrodes were filled with intracellular recording fluid (in $\mathrm{mM}$ : $120 \mathrm{~K}$-gluconate, $20 \mathrm{KCl}, 10 \mathrm{HEPES}, 7 \mathrm{Na}$-Phoscreatine, $4 \mathrm{Na} 2$-ATP, $2 \mathrm{MgCl} 2,0.3$ Tris-GTP, 0.2 EGTA) and used to whole cell patch the neuron.

After establishing stable intracellular and extracellular recordings, the perfusion fluid was switched for 30 seconds to artificial cerebral spinal fluid containing $200 \mu \mathrm{M}$ ouabain. This corresponds approximately to $t=0-30 \mathrm{~s}$ in the recordings. The flow rate of the recording chamber was approximately $4 \mathrm{~mL} / \mathrm{min}$ and its volume $0.5 \mathrm{~mL}$. The applied ouabain concentration, $200 \mu \mathrm{M}$, is two orders of magnitude above the binding constant $K_{d} \approx 50 \mathrm{nM}$ [18], resulting in a complete block of the $\mathrm{Na} / \mathrm{K}$-pumps. A relatively high concentration was chosen to ensure rapid diffusion of the substance into the slice. The washout time of the ouabain was determined to be much longer than the duration of the experiments, on the order of 4-6 hours.

Recordings were amplified using an Axon 700B amplifier (Molecular Devices, Sunnyvale, CA) with a $2.4 \mathrm{kHz}$ low pass filter. Extracellular signals were amplified 500x prior to digitization and intracellular signals were amplified 10x prior to digitization. Analog to digital conversion was performed using a National Instruments (Austin, TX) M-6259 16-bit data acquisition card. Data was recorded using the RealTime eXperiment Interface (RTXI, www.rtxi.org).

Data was analyzed using MATLAB (Natick, MA). The transmembrane voltage signals were obtained by subtracting the extracellular voltage from the intracellular signal. Of the 12 obtained recordings, two were discarded. In one measurement we 
expected a problem with the patch, while the other was halted after $180 \mathrm{~s}$, before the cell fully depolarized. This resulted in 10 measurements for analysis.

Experiments were conducted in accordance to IUCAC approved protocols at the University of Minnesota.

\subsubsection{Analytical methods}

To analyze the observed dynamics, we will use the results of Barreto et al. [17], who characterized a Hodgkin-Huxley $(\mathrm{HH})$ neuron model for different ion concentration gradients. Their analysis was performed to qualitatively study how changes in sodium and potassium Nernst potentials can induce periodic bursting behavior of neurons.

The Nernst potential is a function of the intra- and extracellular ion concentrations, which are not constant in our case. However, since the dynamics of these concentrations are slow compared to the membrane dynamics, the behavior of the membrane voltage can be investigated as though the concentrations are constant. The bifurcation diagram of Barreto et al. [17] depicts the different behaviors of the $\mathrm{HH}$ neuron, e.g. resting, tonically spiking, as a function of both the intracellular sodium concentration and extracellular potassium concentration. In the HH model, these concentrations affect only the corresponding Nernst potentials, $E_{N a}$ and $E_{K}$, which are direct parameters in many single cell models. Therefore, the diagram was redrawn with $E_{K}$ and $E_{N a}$ as parameters, calculated from the concentrations stated in their paper as

$$
E_{x}=27 \mathrm{mV} \cdot \ln \frac{\left[C_{x}\right]_{e}}{\left[C_{x}\right]_{i}} .
$$

Here $\left[C_{x}\right]_{i, e}$ denote, respectively, the intracellular and extracellular concentrations of ion species $x=\mathrm{Na}$, K.

The measurements were grouped in categories based on the dynamic states and transitions that were observed. For example: "from resting state, to tonic spiking starting with zero frequency, to a depolarized resting state through depolarization block", or "depolarization without spiking".

\subsection{Results}

\subsubsection{Membrane voltage}

After the addition of ouabain, the majority of cell membranes depolarized to a resting voltage close to zero, as for example can be seen in Figure 5.1-A1. This is expected, because the main mechanism for maintaining the ion gradients, the sodium-potassium pump, is halted. Interestingly, the cells showed different kinds of dynamical behavior during this process. Five different types of behavior were observed. 

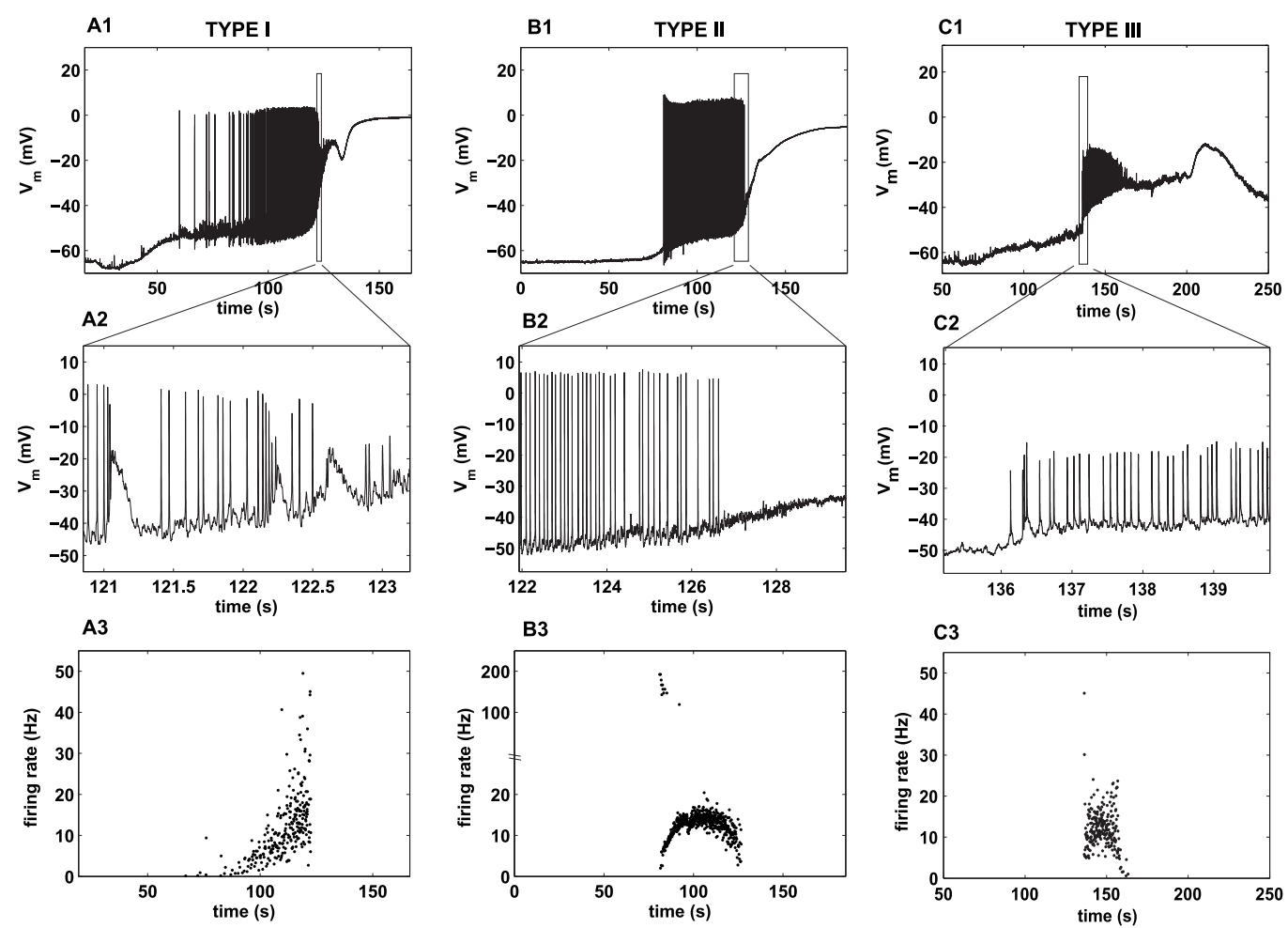

Figure 5.1: Time course of the membrane voltage after 30 s perfusion with $200 \mu M$ ouabain: time traces $(A 1, B 1, C 1)$, a close up of the end $(A 2, B 2)$ or start $(C 2)$ of the spiking epoch and the firing rates, calculated from the inter-spike intervals (A3, B3, C3). Panels A show an example of the first type of dynamical behavior described in the Results, where spiking is stopped by a depolarization block, panels B present the second type, where spiking stops by slowing down and panels $C$ show the third type, where the spikes have a small amplitude. 

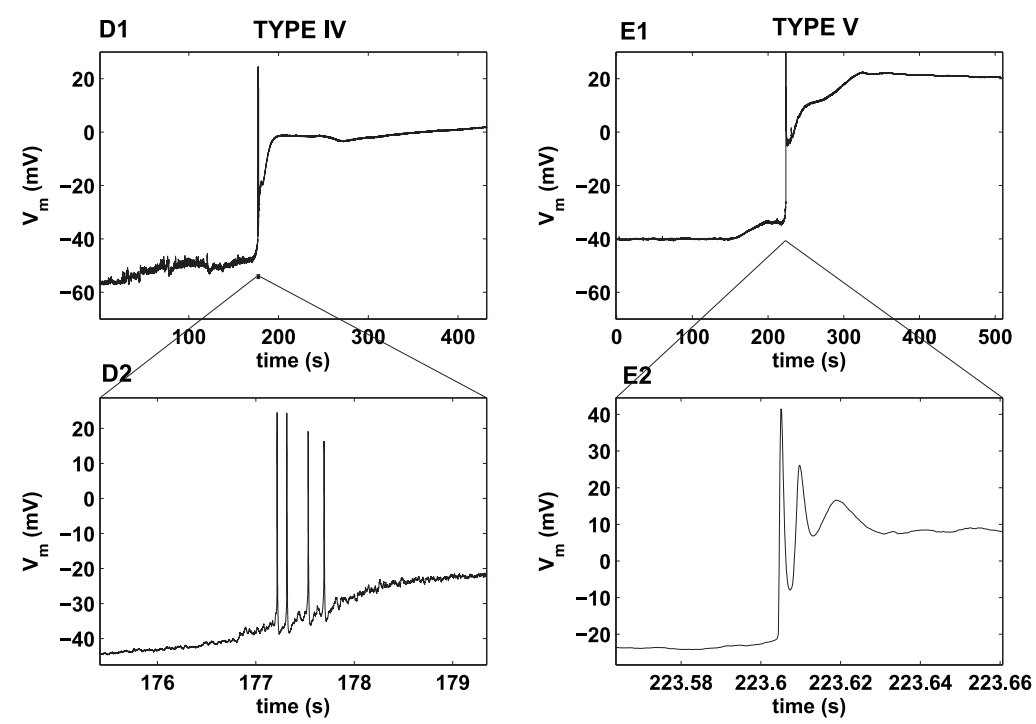

Figure 5.2: Time course of the membrane voltage after 30 s perfusion with $200 \mu M$ ouabain: time traces $(D 1, E 1)$ and a close up (D2, E2). Panels D show the fourth type of dynamical behavior described in the Results, in which only a few or no spikes occur. Panels E show the fifth type, where the membrane voltage shows a relaxation oscillation from a polarized to a depolarized state. Note the short timescale on the time axis for this close up.

Figure 5.1-A shows an example of the first type, recorded in two out of ten measurements. After the application of ouabain, the cell's membrane potential increases from about $-65 \mathrm{mV}$ to $-55 \mathrm{mV}$ ( $\mathrm{t}=20$ to 60 seconds). In the next 30 seconds, $V_{m}$ shows large subthreshold variations and a few action potentials. This suggests that the cell receives substantial synaptic input. The height of the voltage plateau does not change significantly during this period. Then, from 90-120 s, the voltage plateau depolarizes and the spiking frequency increases. The neuron achieves a maximal firing rate of $50 \mathrm{~Hz}$, just before going into depolarization block. In the case shown, the neuron goes into block and back to spiking several times. After the cell goes into block the last time, it has a membrane voltage around $-20 \mathrm{mV}$. Then, in approximately 30 seconds, it depolarizes and finally reaches a membrane potential of approximately 0 $\mathrm{mV}$.

In four recordings, labeled as type 2, we observed a spiking epoch with a similar onset, but with a different ending, as shown in Figure 5.1-B. Here, the fastest firing rate occurs in the middle of the spiking period. The cell does not go into depolarization block, but decreases its spiking frequency to zero. Then the membrane depolarizes from about $-40 \mathrm{mV}$ up to its final potential in approximately 30 seconds.

Furthermore, panel B3 shows the occurrence of two-spike bursts, with an interspike interval $<10 \mathrm{~ms}(>100 \mathrm{~Hz})$. The bursts are followed by after-spike hyperpo- 
larizations, which are deeper than those following single spikes. These bursts were observed in several of the recordings labeled as type 1 or 2 .

Labeled as type 3, shown in Figure 5.1-C, one recording displayed a spiking epoch with a frequency decreasing towards zero, as the second type, but the onset of spiking was not at almost zero frequency, but had an initial spike rate of about 7 Hz. The amplitude of the spikes is small and the minimum voltage during spiking is above the resting state voltage.

Two recordings, that were labeled as the fourth type, did not exhibit a spiking epoch. No, or only a few, spikes are observed before the neuron depolarizes in a similar way as the second type. Figure 5.2-D shows one recording as an example. The second recording was similar, but did not exhibit any action potentials.

A fifth type was observed in one recording, shown in Figure 5.2-E: a single spike followed by a relaxation oscillation to a depolarized membrane voltage.

\subsubsection{Analysis of the membrane voltage dynamics}

We will use bifurcation analysis to explain the different observed dynamics. Bifurcations are the points in parameter space where the system changes behavior, for example from resting to tonic spiking. The bifurcation analysis performed by Barreto et al. [17] of a Hodgkin-Huxley model, determines the dynamics of a neuron for different ion concentrations. Figure 5.3 shows this bifurcation diagram.

The saddle-node-on-invariant-circle (SNIC) bifurcation line depicts the points where the neuron changes its behavior from resting to tonic spiking. When either $E_{K}$ or $E_{N a}$ increases (moving right or down in Figure 5.3), the net inward current is increased, depolarizing the neuron. Upon crossing the bifurcation line the resting state vanishes, which causes the neuron to tonically spike, starting at zero frequency. To the left of this curve the neuron is excitable, the closer to the line, the more excitable. To the right of this line, the neuron tonically spikes, with increasing frequency as the system moves further from the bifurcation line. This is known as class 1 excitability [19].

The Hopf bifurcation line corresponds to the points where the neuron goes into depolarization block. Such a block occurs when the spiking frequency becomes so high that the sodium gates have no time to deinactivate completely after an action potential. This reduces the peak amplitude of the action potential, which in turn decreases the opening of the potassium gates and the resulting repolarization. Upon crossing the line, the amplitude of the action potential becomes zero [19]. Hence the membrane voltage oscillates towards a stable depolarized state. The sodium and the potassium gates are then half open and the electrical membrane resistance is low [20].

A saddle-homoclinic orbit (HC) and two saddle-node ( $\mathrm{SN}$ ) bifurcations, connect the SNIC and Hopf lines. Upon crossing the HC bifurcation, the potassium current 


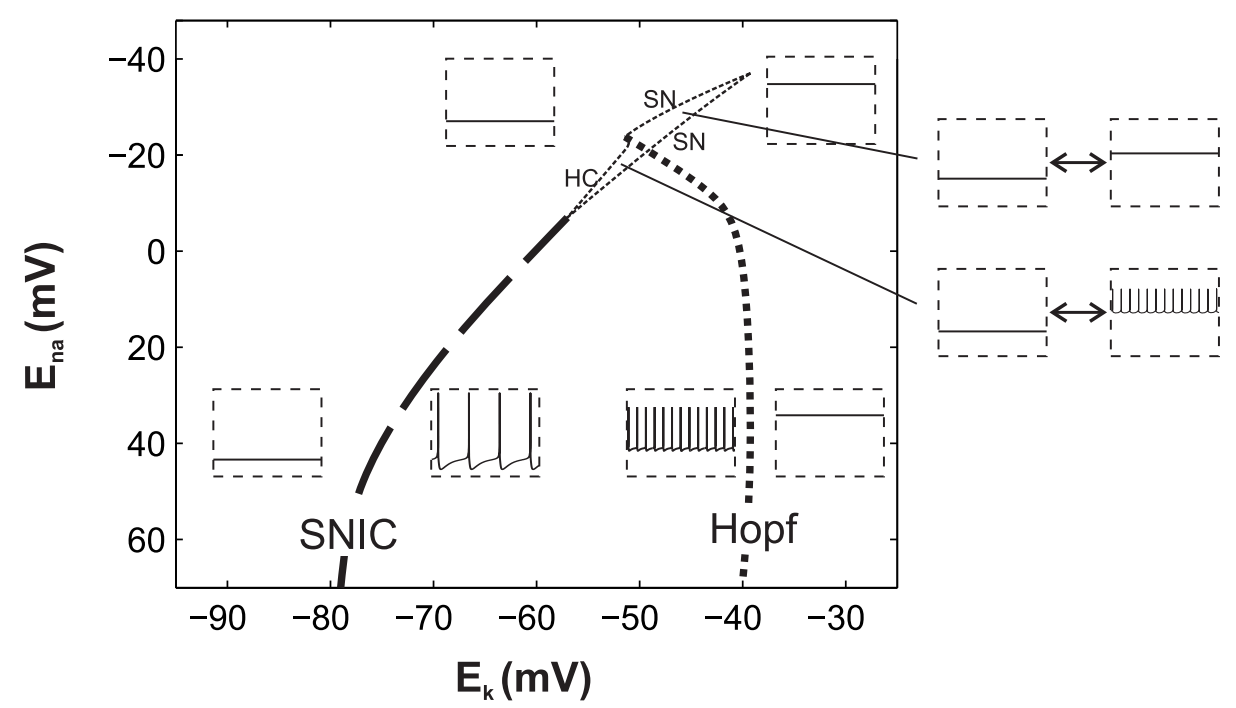

Figure 5.3: Sketch of a bifurcation diagram of a Hodgkin-Huxley type model. Shown are the SNIC bifurcation (thick dashed line), which corresponds to a class 1 spiking threshold, and the Hopf bifurcation (thick dotted line), which corresponds to a depolarization block. A saddle-homoclinic orbit (HC) and two saddle-node (SN) bifurcations (thin dashed lines) connect the SNIC and Hopf lines. The insets depict qualitatively the dynamics of the membrane voltage at different positions in the diagram. In the small area between the HC and SN bifurcation lines, the neuron dynamics are bistable (denoted by double-headed arrows). Bifurcation lines are calculated from the diagram depicted in [17]. 
has become too small to fully repolarize the membrane voltage to the resting state once an action potential is induced, and the cell keeps spiking. The neuron has become bistable and input can switch it between spiking and resting. The bifurcation can be identified from the lack of hyperpolarization below the resting voltage, when the cell is generating action potentials.

When instead the left SN bifurcation line is crossed, a stable depolarized equilibrium is formed and the cell can be switched between the polarized resting state and the depolarized resting state. When the system subsequently crosses the right SN line, the polarized resting state loses stability.

We now show that a single cell, with the same electrophysiological membrane properties, can exhibit any of the five types of the experimentally observed dynamics, depending on the trajectory that is followed in parameter space by the sodium and potassium Nernst potentials, $E_{N a}$ and $E_{K}$. Under physiological circumstances these potentials are stable around 50 and $-90 \mathrm{mV}$ respectively. When all ion pumps stop functioning, the ion gradients diminish and these voltages will go towards the socalled Donnan equilibrium [1]. The ion gradients do not completely vanish, due to charged, large molecules in the intracellular space and gradients may even reverse. In our experiments, the chloride pumps are still functioning, which also keeps the equilibrium potentials from zero.

In Figure 5.4, hypothetical trajectories are drawn that correspond to the experimentally observed behaviors. Both trajectories 1 and 2 cross the SNIC bifurcation, transitioning from resting to regularly spiking. Trajectories 4 and 5 do not cross this bifurcation. In trajectory 1 , the potassium reversal potential is lost faster than that of sodium and the cell goes through the Hopf bifurcation into depolarization block. In trajectory 2, 4 and 5, the sodium reversal potential is lost faster than that of potassium and the cell exits the spiking regime via the SNIC bifurcation and firing rate slows down to zero (trajectory 2), or the trajectory stays above the SNIC bifurcation and the cell does not spike at all (trajectories 4 and 5).

For types 2 and 4 no further bifurcations occur and the membrane depolarizes due to loss of the potassium gradient. For type 5, the trajectory crosses two saddle-node bifurcations. This causes a depolarized equilibrium to stabilize, followed by the loss of stability of the physiological resting equilibrium. This leads to a fast relaxation oscillation to the depolarized equilibrium. The trajectory of type 3 (not shown in Figure 5.4), lies slightly below trajectory 5 and crosses the homoclinic orbit bifurcation. This leads to a short period of spiking, that starts at a non-zero frequency and shows small action potentials that are not followed by a hyperpolarization. Because the spiking frequency decreases to zero, it can be concluded that, rather than moving through the Hopf bifurcation, the trajectory crossed the HC bifurcation again before the membrane completely depolarized.

These five trajectories produce all dynamic transitions that can occur when the 


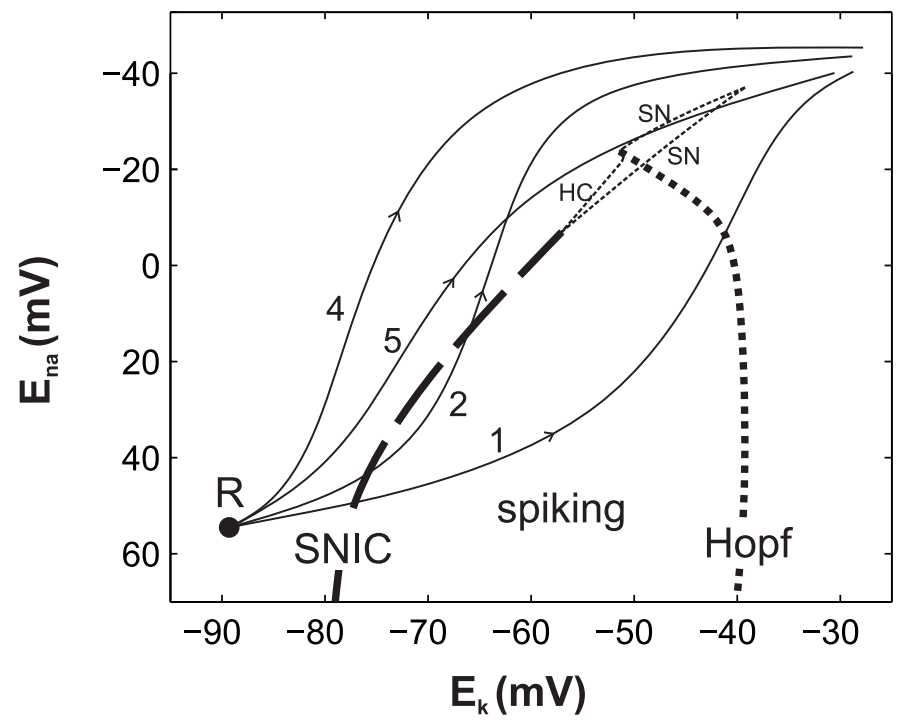

Figure 5.4: Trajectories through the bifurcation diagram of a Hodgkin-Huxley type model. The physiological state is denoted $R$. Solid lines denote four hypothetical trajectories of the ion concentrations, leading to the experimentally observed dynamics. Trajectory 3 (not shown for clarity) lies slightly below trajectory 5 and crosses the HC bifurcation. Bifurcation lines are calculated from the diagram depicted in [17].

sodium and potassium gradients change monotonically in this model. As comparison, Figure 5.5 shows the behavior of the HH model in which the Nernst potentials were set to follow these trajectories. The behavior largely agrees with that experimentally observed.

\subsection{Discussion and conclusion}

We conducted this study to improve our understanding of the dynamics of a neural unit during anoxic depolarizations. Here, we focused on the membrane voltage. The membrane voltage of pyramidal cells from rat neocortex was measured after blocking the sodium-potassium pumps with ouabain. This induced anoxic-like depolarizations. Five different kinds of dynamic behavior were observed. Bifurcation analysis shows that these correspond to different paths through the state space of the sodium and potassium Nernst potentials of the same neuron model.

To our knowledge, this is the first time this variety in voltage dynamics of depolarizing neurons is experimentally observed. The observed types are characterized by the bifurcations through which the system goes, which are shown to be the bifurcations that occur in the HH-model. All the bifurcations predicted by the HH-model, 


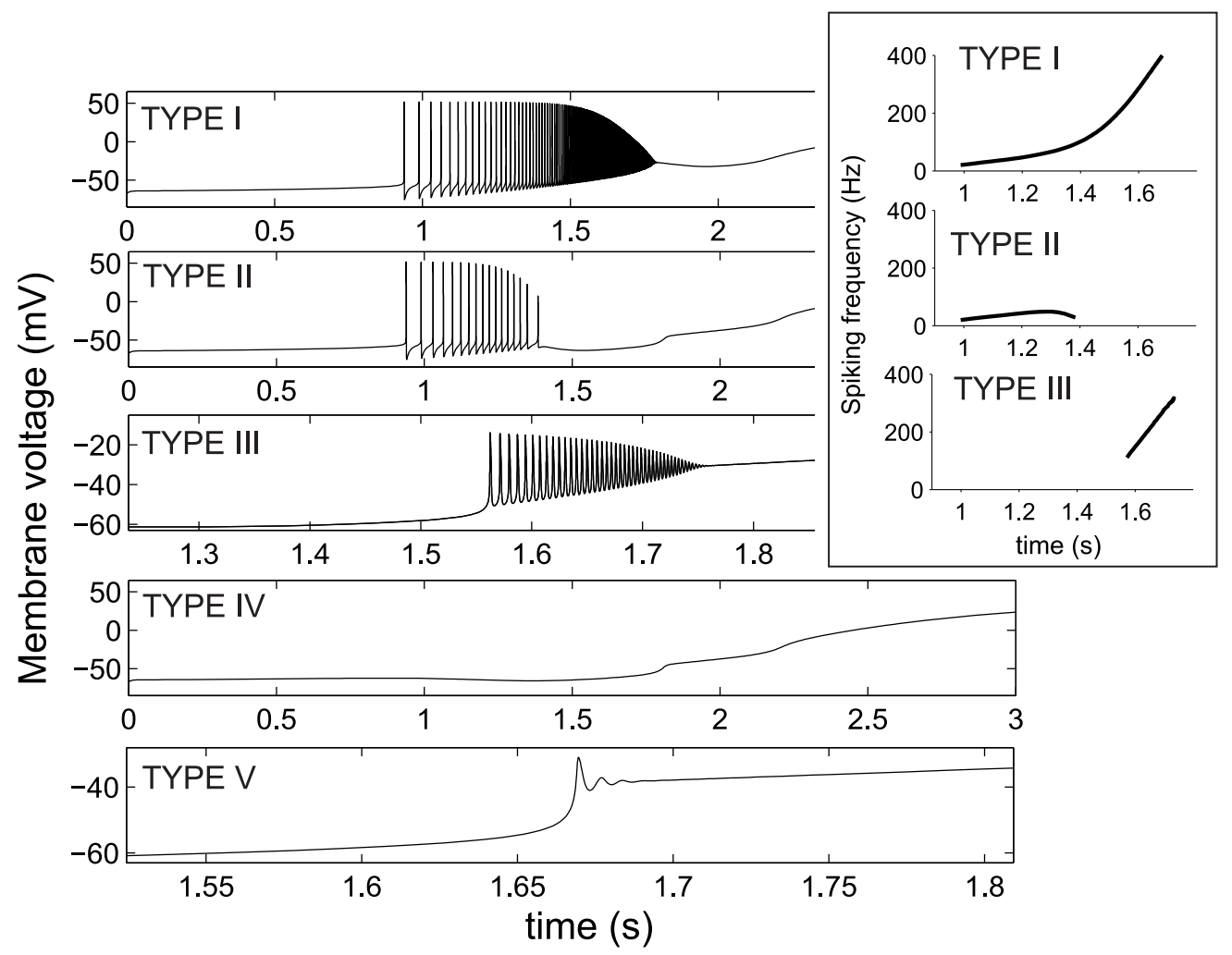

Figure 5.5: Membrane voltage dynamics of the $\mathrm{HH}$ model for five different trajectories of the Nernst potentials. The inset shows the spiking frequency. The time courses of $E_{K}$ and $E_{N a}$ were set to follow approximately the trajectories in Figure 5.4. Since the HH model shows a much higher firing rate than the measured neurons, the Nernst potentials were changed on a much shorter time scale (4s) than experimentally observed (minutes), in order to show the depolarization and the spiking behavior in the same plot: $E_{K}=-90+65 *(\tanh (t-2)+1)$ and $\left.E_{N a}=53+\Delta E_{N a} \tanh \left(\left(E_{K}-\mu\right) / w\right)+1\right) / 2$, using $\left[\Delta E_{N a}, \mu, w\right]=[103,-40,10 ; 103,-60,5 ; 68,-$ $70,10 ; 103,-70,10 ; 82,-70,10] \mathrm{mM}$, respectively. Parameters of the voltage gated channels were chosen as in [17]. 
but not all possible combinations, were observed in the measurements. The bifurcation diagram suggests that other types of dynamics can occur that are combinations of the observed dynamics. For example, the spiking epoch of type 2 could be followed by the relaxation oscillation of type 5 , and the epoch of low amplitude spikes of type 3 could end with the Hopf bifurcation shown by type 1, instead of slowing down in spike rate (c.f. Figures 5.1-C and 5.5-3). From the limited amount of measurements (10), however, we cannot exclude the existence of behavior not predicted by the HH-model. Furthermore, neurons with spiking dynamics that are not described by this HH-model, such as intrinsically bursting cells, will certainly show different membrane dynamics during depolarization.

Some of the voltage dynamics, for example those in Figure 5.1-C, may appear atypical. They do not, however, indicate a diseased state of the cell, but are rather the normal response of the membrane voltage to particular courses of the intra- and extracellular ion concentrations. When performing experimental measurements, classifying the membrane dynamics during the depolarizations can yield additional information on the changes in the sodium and potassium gradients.

The dynamics of the ion concentrations are a complex function of various parameters, including cell volume, membrane area and extracellular volume, local glial cell activity, the effective distance for diffusion between the cell and the bath fluid, and the ion channel densities of the cells $[3,4]$. Furthermore, the bifurcation diagram itself depends on the cell's channel conductances and the synaptic input it receives, which is different from cell to cell. This may account for the differences between the measurements.

Since the synapses were fully functional during the measurements, they affect the membrane dynamics. Synaptic input can shift the positions of the bifurcations, but does not qualitatively change the structure of the bifurcation diagram. Therefore, only the fluctuations in synaptic input can be observed from the membrane voltage trace, showing as subthreshold movements, variations in the spike intervals or the occurrence of a few spikes (e.g. Figures 5.1-A1 and 5.2-D2). When the neuron is close to a bifurcation, these fluctuations can even cause transitions back and forth between states, as for example observed in Figure 5.1-A2, where the dynamics fluctuate between spiking and depolarization block.

During our analysis of the single neuron dynamics, we considered the courses of the potassium and sodium Nernst potentials as given. Also, in order to replicate the dynamics observed in the measurements with the HH-model, trajectories in the state space of the Nernst potentials were artificially chosen. In reality, however, these trajectories are determined by the closed-loop interaction between the transmembrane ion fluxes resulting from neuronal activity and the dynamics of the ion concentrations. This interaction determines the critical conditions for triggering a rapid depolarization, such as a potassium concentration threshold or minimum ion pump activity. 
Further efforts are needed to experimentally verify this interaction, as modeled in $[2,17]$, as well as the influence of synaptic coupling between the cells. This requires simultaneous measurement of the ion concentrations, the membrane voltage and neural activity. This may also allow a deeper understanding of clinically relevant phenomena, i.e. the occurrence of (spreading) depolarizations in migraine or ischemia/anoxia. Indeed, to identify the conditions responsible for these mesoscopic events, the dynamics of the ion concentrations must be considered as well.

Trajectories in the bifurcation diagram derived from the calculations of Barreto et al. [17] must reach a sodium Nernst potential $<-20 \mathrm{mV}$ in order to circumvent the Hopf bifurcation line, and hence produce a depolarization without a depolarization block (Trajectories 2-5). This is not likely, as the intracellular sodium concentration would have to become almost twice as large as the extracellular sodium concentration. However, the positions of the bifurcations depend heavily on the values for the conductances and the specific model used. Krishnan et al. [7], for example, constructed a similar, more elaborate model, using values of mammalian cortical cells. Their bifurcation diagram is qualitatively similar, but they find the highest point of the spiking regime at a sodium Nernst potential of $47 \mathrm{mV}$, rather than $-20 \mathrm{mV}$. This sodium potential can certainly be reached during anoxic depolarization. So, although a mammalian neuron is more complex than the $\mathrm{HH}$ model and the values at which the bifurcations occur in the model will not be commensurate with the experimental findings, the model is able to explain the bifurcations. Therefore, our observations agree very well with the model's qualitative behavior.

We studied membrane dynamics after failure of the $\mathrm{Na} / \mathrm{K}$-pumps, by blocking them with ouabain. The results may therefore be slightly different from in vivo pathophysiology, where other processes play a role as well. During OGD, for example, synaptic transmission is one of the first processes to fail [21]. Furthermore, the chloride pumps stop functioning, which results in a hyperpolarizing chloride current [22]. Also, ATP-sensitive potassium channels can open [23]. Due to these effects, neurons tend to temporarily hyperpolarize during OGD. Therefore, it is expected that during OGD the onset of depolarization will be later then after application of ouabain. However, since the gated currents are larger than the currents generated by these effects, the dynamic behavior during the depolarization is expected to be similar.

Our results may also be relevant to epilepsy and seizure generation. It is known that an increase of extracellular potassium, in the range observed in vivo during seizures, depolarizes cortical pyramidal neurons resulting in increased excitability. Failure in maintaining homeostasis of the extracellular potassium concentrations has therefore been proposed to play a significant role in seizure generation and termination [24]. Our results help identify different "ionic routes" to depolarization block in seizures. Different rates of accumulation of extracellular potassium and intracellular sodium may determine different seizure dynamics. This may be useful in classifying 
seizure types and determining which drug targets may be more effective depending on the seizure dynamics.

To conclude, we report on experimental observations on single neurons during anoxic-like depolarizations. In these measurements, five different types of dynamics were observed. These are explained with a bifurcation diagram of a single cell model and were shown to correspond to different courses of the sodium and potassium gradients. All dynamical states and transitions predicted by the model were observed. This shows that bifurcation analysis of single cell models for the sodium and potassium Nernst potentials is indeed a successful approach to understand the membrane voltage dynamics during anoxic and anoxic-like depolarizations, that can occur during epilepsy, migraine and stroke.

\section{References}

[1] G. G. Somjen, Ions in the Brain - Normal Function, Seizures, and Stroke, chapter 18 (Oxford University Press) (2004).

[2] B.-J. Zandt, B. ten Haken, J. G. van Dijk, and M. J. A. M. van Putten, "Neural dynamics during anoxia and the "wave of death"”, PLoS One 6, e22127 (2011).

[3] J. R. Cressman, G. Ullah, J. Ziburkus, S. J. Schiff, and E. Barreto, "The influence of sodium and potassium dynamics on excitability, seizures, and the stability of persistent states: I. single neuron dynamics.", J Comput Neurosci 26, 159-170 (2009).

[4] J. R. Cressman, G. Ullah, J. Ziburkus, S. J. Schiff, and E. Barreto, "Erratum to: The influence of sodium and potassium dynamics on excitability, seizures, and the stability of persistent states: I. single neuron dynamics", J Comput Neurosci 30, 781-781 (2011).

[5] H. Kager, W. J. Wadman, and G. G. Somjen, "Simulated seizures and spreading depression in a neuron model incorporating interstitial space and ion concentrations.", J Neurophysiol 84, 495 512 (2000).

[6] A. Bragin, M. Penttonen, and G. Buzski, "Termination of epileptic afterdischarge in the hippocampus.”, J Neurosci 17, 2567-2579 (1997).

[7] G. P. Krishnan and M. Bazhenov, "Ionic dynamics mediate spontaneous termination of seizures and postictal depression state.", J Neurosci 31, 8870-8882 (2011).

[8] M. A. Kramer, W. Truccolo, U. T. Eden, K. Q. Lepage, L. R. Hochberg, E. N. Eskandar, J. R. Madsen, J. W. Lee, A. Maheshwari, E. Halgren, C. J. Chu, and S. S. Cash, "Human seizures self-terminate across spatial scales via a critical transition.”, Proc Natl Acad Sci U S A 109, 21116-21121 (2012).

[9] J. P. Dreier, "The role of spreading depression, spreading depolarization and spreading ischemia in neurological disease.”, Nat Med 17, 439-447 (2011).

[10] B.-J. Zandt, B. Ten Haken, and M. J. A. M. van Putten, "Diffusing substances during spreading depolarization: Analytical expressions for propagation speed, triggering, and concentration time courses.", J Neurosci 33, 5915-5923 (2013). 
[11] B. E. Lindquist and C. W. Shuttleworth, "Adenosine receptor activation is responsible for prolonged depression of synaptic transmission after spreading depolarization in brain slices.", Neuroscience 223, 365-376 (2012).

[12] H. Nakamura, A. J. Strong, C. Dohmen, O. W. Sakowitz, S. Vollmar, M. Su, L. Kracht, P. Hashemi, R. Bhatia, T. Yoshimine, J. P. Dreier, A. K. Dunn, and R. Graf, "Spreading depolarizations cycle around and enlarge focal ischaemic brain lesions.”, Brain 133, 1994-2006 (2010).

[13] M. Lauritzen, J. P. Dreier, M. Fabricius, J. A. Hartings, R. Graf, and A. J. Strong, "Clinical relevance of cortical spreading depression in neurological disorders: migraine, malignant stroke, subarachnoid and intracranial hemorrhage, and traumatic brain injury.", J Cereb Blood Flow Metab 31, 17-35 (2011).

[14] R. Hauger, H. M. Luu, D. K. Meyer, F. K. Goodwin, and S. M. Paul, "Characterization of "highaffinity" [3h]ouabain binding in the rat central nervous system.", J Neurochem 44, 1709-1715 (1985).

[15] W. R. Schlue, "Effects of ouabain on intracellular ion activities of sensory neurons of the leech central nervous system.", J Neurophysiol 65, 736-746 (1991).

[16] W. Sandtner, B. Egwolf, F. Khalili-Araghi, J. E. Snchez-Rodrguez, B. Roux, F. Bezanilla, and M. Holmgren, "Ouabain binding site in a functioning nat/k+ atpase.", J Biol Chem 286, 38177-38183 (2011).

[17] E. Barreto and J. R. Cressman, "Ion concentration dynamics as a mechanism for neuronal bursting.", J Biol Phys 37, 361-373 (2011).

[18] A. A. Maki, D. G. Baskin, and W. L. Stahl, "[3h]-ouabain binding sites in rat brain: distribution and properties assessed by quantitative autoradiography.", J Histochem Cytochem 40, 771-779 (1992).

[19] E. M. Izhikevich, Dynamical Systems in Neuroscience: The Geometry of Excitability and Bursting (Computational Neuroscience), 1 edition (The MIT Press) (2006).

[20] D. Bianchi, A. Marasco, A. Limongiello, C. Marchetti, H. Marie, B. Tirozzi, and M. Migliore, "On the mechanisms underlying the depolarization block in the spiking dynamics of cal pyramidal neurons.”, J Comput Neurosci 33, 207-225 (2012).

[21] J. Hofmeijer and M. J. A. M. van Putten, "Ischemic cerebral damage: an appraisal of synaptic failure.", Stroke 43, 607-615 (2012).

[22] M. Müller, "Effects of chloride transport inhibition and chloride substitution on neuron function and on hypoxic spreading-depression-like depolarization in rat hippocampal slices.", Neuroscience 97, 33-45 (2000).

[23] M. Müller, J. Brockhaus, and K. Ballanyi, "Atp-independent anoxic activation of atp-sensitive k+ channels in dorsal vagal neurons of juvenile mice in situ.", Neuroscience 109, 313-328 (2002).

[24] F. Frhlich, M. Bazhenov, V. Iragui-Madoz, and T. J. Sejnowski, "Potassium dynamics in the epileptic cortex: new insights on an old topic.”, Neuroscientist 14, 422-433 (2008). 


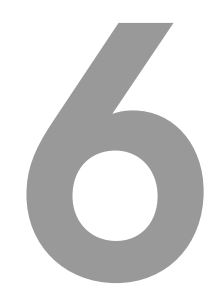

\title{
A neural mass model based on single cell dynamics to model pathologies *
}

\begin{abstract}
Neural mass models are successful in modeling brain rhythms as observed in macroscopic measurements such as the electroencephalogram (EEG). While the synaptic current is explicitly modeled in current models, the single cell electrophysiology is not taken into account. To allow for investigations of the effects of channel pathologies, channel blockers and ion concentrations on macroscopic activity, we formulate neural mass equations explicitly incorporating the single cell dynamics. The mean and variance of the firing rate and synaptic input distributions are modeled. The firing rate curve (F(I)-curve) is used as link between the single cell and macroscopic dynamics. We show that this model accurately reproduces the behavior of two populations of synaptically connected Hodgkin-Huxley neurons, also in non-steady state.
\end{abstract}

*Submitted as: BJ Zandt, S Visser, MJAM van Putten, B ten Haken (2014) A neural mass model based on single cell dynamics to model pathologies. 


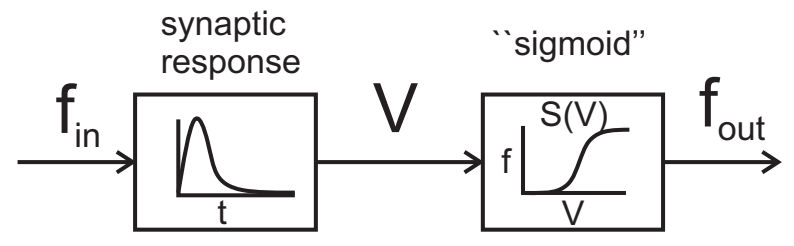

Figure 6.1: Diagram of a neural mass model describing one population with one input. The input to a population $f_{\text {in }}$, i.e. the presynaptic firing rate, drives the dynamics of the state of the population, represented by the population potential $V$. The response of $V$ to $f_{\text {in }}$ represents the dynamics of the synapses and the membrane potential of the dendrites and soma. This state is consequently converted into the output firing rate $f_{\text {out }}$ by a sigmoidal function $S(V)$.

\subsection{Introduction}

Neural mass models (NMM) are very successful in describing brain rhythms as measured with electroencephalogram (EEG), electrocorticogram (ECoG) and magnetoencephalogram (MEG). The main advantage of these models is that they can be mathematically analyzed due to their low dimensionality and that they are computationally inexpensive. In short, a NMM produces the average activity (firing rates) of populations of neurons [1]. If desired, NMM's can be extended with a spatial component (neural field) to model the propagation of rhythms and activations.

Neural mass models can also be used to investigate how (patho)physiological changes in the brain affect EEG rhythms. For example, they were recently used to show how the effects of anesthetics/sedatives [2-4] and selective synaptic damage [5] result in the patterns observed in EEG's from patients under sedation and with ischemic damage, respectively.

The models contain explicit expressions for the synaptic response functions (see figure 6.1). Therefore, it is clear how to incorporate factors that alter the synaptic responses. For instance, propofol prolongs this response, which can be incorporated in a NMM by increasing the synaptic time constants [2]. However, an explicit relation between the sigmoidal function and the dynamics of single cells is missing. Therefore, it is not trivial how to correctly adapt the sigmoid to model the EEG of patients with conditions that alter the dynamics of the individual neuron, like those induced by voltage-gated channel blockers, channel mutations or changes in ion concentrations. Such factors play an important role in the (patho)physiology of patients with ischemia, head trauma, epilepsy and migraine [6-9].

In this paper we aim to provide a straight-forward description that relates a NMM to the dynamics of the single cells and their connectivity. From this description, we construct a new neural mass model. In particular, we investigate the relation of the sigmoid to the single cell dynamics and their connectivity, by describing the dynamics of a network of excitatory and inhibitory conductance based cells (see figure 6.2). 
Network of single cell models

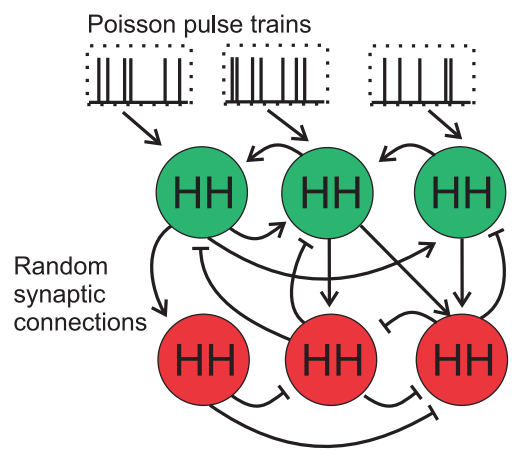

Neural mass model

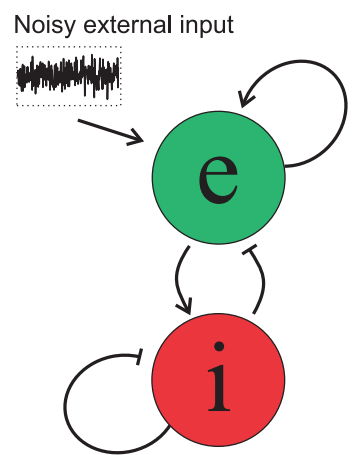

Figure 6.2: The approach we use for modeling neural dynamics. A network of spiking neurons is taken as starting point (left). It consists of two populations of synaptically coupled Hodgkin-Huxley $(\mathrm{HH})$ neurons. The new model we will construct, a neural mass model (right), describes the average dynamics of each population.

We confirm that our newly proposed neural mass model accurately reproduces the dynamics observed in a network of spiking neurons.

To take the influence of the connectivity in the network into account, we study the distributions of firing rates and input currents of the neurons. The dynamic evolution of these distributions is then expressed up to second order, i.e. mean and variance. The distribution of input currents yields, together with the F(I)-curves, the distribution of firing rates within the population. Changes in electrophysiological parameters of the cells typically shift and/or stretch these F(I)-curves. The well-known Hodgkin-Huxley $(\mathrm{HH})$ model is used as single cell model because it is the simplest model which is fully expressed in physiological parameters. Our approach is general, though, and allows for relating a neural mass model to networks of other single cell spiking models as well.

First, we discuss the interpretation of the variables which commonly appear in a neural mass model and some critical issues with the classical derivation of the sigmoidal function. Then we describe our NMM that includes the single cell dynamics, its implementation in Matlab, and validate this new model with a network of spiking cells.

\subsubsection{Neural mass modeling}

We discuss the typical parameters and variables of a neural mass model, and their interpretation. The model from Liley et al. [10] is used as example.

The variable representing the state of the population is the population potential $V$ (denoted $h$ in [10]). The EEG signal is assumed to be proportional to $V$. It is 
interpreted as the average membrane potential of the cells' somas. $V$ is obtained by low-pass filtering the input spike rate to the population with a set of differential equations. Their impulse response is the postsynaptic potential (PSP) of a neuron, i.e. the response of the post-synaptic conductance and subsequently the post-synaptic membrane voltage to a presynaptic spike:

$$
\begin{aligned}
& I_{\mathrm{syn}, \mathrm{x}}=H * f_{x} \\
& \tau \frac{d V}{d t}=V_{\text {rest }}-V+\sum_{x} \psi I_{\mathrm{syn}, \mathrm{x}},
\end{aligned}
$$

where $x$ denotes the populations from which input is received (i.e. excitatory and inhibitory population), $H * f_{x}$ is the synaptic impulse response convoluted with the presynaptic firing rate, $\tau$ the membrane time constant, $V_{\text {rest }}$ the resting potential and $\psi$ is the synaptic effectiveness. This effectiveness depends weakly on $V . I_{\mathrm{syn}, \mathrm{x}}$ are the synaptic activations of the synapses originating from the two populations respectively. Liley et al. chose alpha synapses in their model, for which the convolution with $H$ corresponds to a second order differential equation.

Subsequently, $V$ is converted to an output firing rate of the population through a sigmoidal function $S(V)$. A symmetric sigmoid is derived under the assumption that a cell is either active (firing) or not, and a cell is assumed active when its membrane potential is above its spiking threshold. Furthermore it is assumed that either all cells receive the same input, but have different voltage thresholds, or the variation in spiking threshold is relatively small, while the variation in input or noise causes cells to have different membrane potentials. These two interpretations are visualized in figure 6.3. When Gaussian distributions are assumed, both assumptions result in a sigmoid that is the integral of a Gaussian curve [1]. For simplicity, this integral is often approximated by:

$$
S(V)=\int_{-\infty}^{V} \frac{1}{\sigma \sqrt{2 \pi}} \exp \left\{\frac{\left(V^{\prime}-\mu\right)^{2}}{2 \sigma^{2}}\right\} \mathrm{d} V^{\prime} \approx \frac{f_{\max }}{1+\exp (\sqrt{2}(\mu-V) / \sigma)},
$$

where $\mu$ is the threshold voltage, $\sigma$ the width of the voltage distribution and $f_{\max }$ the firing rate of a single neuron when active, i.e. the maximum mean firing rate of the population.

These derivations assume a constant firing rate of the cells when above threshold (so-called McCulloch-Pitts neurons), contrary to the fact that neurons have an increasing firing rate for increasing input currents. Still, the models using this sigmoid are successful, because they are operated on only a small part of the $f=S(V)$ curve, with parameters that are fitted to reproduce experimental data. Therefore, an exact determination of the entire sigmoidal firing rate curve from a physiological basis is often not necessary to obtain realistic results. This means that the sigmoidal 

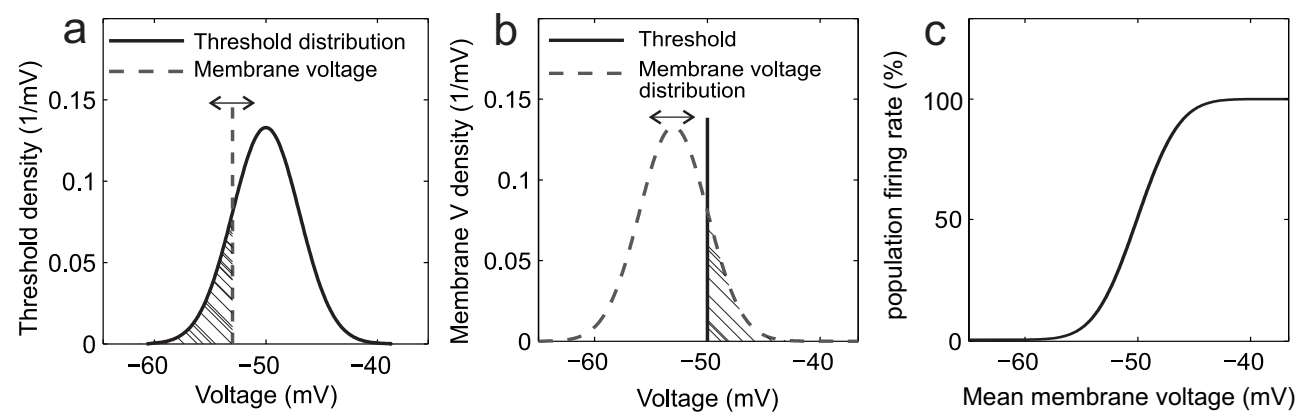

Figure 6.3: Visualization of calculating the sigmoidal voltage-to-spike-rate function (c), from either an assumed Gaussian distribution of the spiking thresholds (a) or the induced membrane voltages of the neurons (b). The hatched area is the part of the population that has a membrane voltage above threshold and is considered to be spiking. The double headed arrow denotes that the average membrane voltage can vary in time.

curve is de facto phenomenological, despite the fact that its parameters suggest to be physiological.

Another issue is that the interpretation of "the membrane voltage of a cell" is not clear in the derivations above, especially not for spiking cells. Two interpretations are [11]: 1, the hypothetical membrane potential of the somas, if they had been passive (linear). The somas with a potential above threshold are actually spiking. 2, the postsynaptic membrane voltage of the dendritic cable. In this case, the dendritic membrane voltage induces an input current into the soma that, when large enough, generates action potentials. It can be shown that the reaction time of the population is too slow when using the first interpretation of passive somas [11]. Therefore we will adhere to the second interpretation. This means that $V-V_{\text {rest }}$ is interpreted as a measure for the input current a cell receives, following Ohms law.

For a more in-depth discussion of the derivation of neural mass models, we recommend the excellent review from Deco et al. [12].

\subsection{Constructing a new neural mass model}

We will derive the average behavior of a population of neurons from the properties of a network of synaptically connected cells, by describing the statistics of the synaptic inputs and the firing rates. The sigmoidal function will be derived using both the $\mathrm{F}(\mathrm{I})$-curve of the single cells and the variance of the input current over the cells. Therefore, we need to calculate not only the mean input and output, but also their variances. Note that throughout this work the terms mean and variance denote the instantaneous distribution over the cells in a population, i.e. the ensemble, not a distribution over time. 


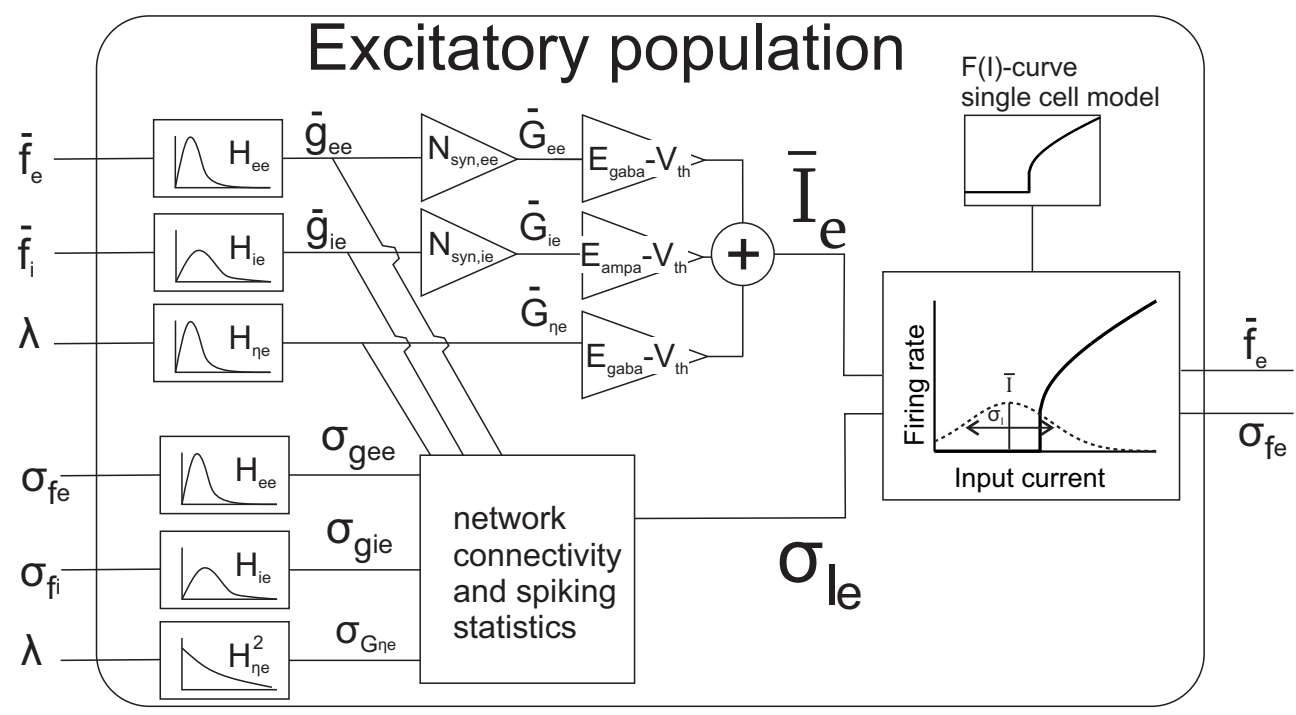

Figure 6.4: Schematic of the excitatory population in the neural mass model. The population receives input from the excitatory and inhibitory populations, as well as excitatory external input $\lambda$, with firing rates with mean $\bar{f}$ and variance $\sigma_{f}$. These are convoluted with their synaptic responses to yield the conductances of the single synapses (mean $\bar{g}$ and variance $\left.\sigma_{g}\right)$. These are the state variables in the model. From these the input currents are calculated (mean $\bar{I}$ and variance $\sigma_{I}$ ). Together with the single cell $F(I)$-curve, they produce the output firing rates.

Figure 6.4 shows schematically our model for the excitatory population and the synapses that project onto these cells. The schematic for the inhibitory population is similar, but without external input $\lambda$. The excitatory population (e) receives excitatory input from itself and from an external source $(\eta)$, and inhibitory input from the inhibitory population (i). The mean $\bar{f}$ and variance $\sigma_{f}$ of the firing rates of the three input sources are convoluted with the corresponding impulse response $H$. This yields the mean $\bar{g}$ and variance $\sigma_{g}$ of the corresponding synaptic conductances. These are the dynamical state variables in our model. These in turn are combined into the mean $\bar{I}$ and variance $\sigma_{I}$ of the input currents into the cells, from which the mean and variance of the output firing rates are calculated. We derive the model step by step, starting with the output.

\subsubsection{The firing rate of a population: the sigmoid function}

In this section we derive a sigmoidal function from the distribution of input currents. We assume these to be normally distributed, since the input current of each cell is the 

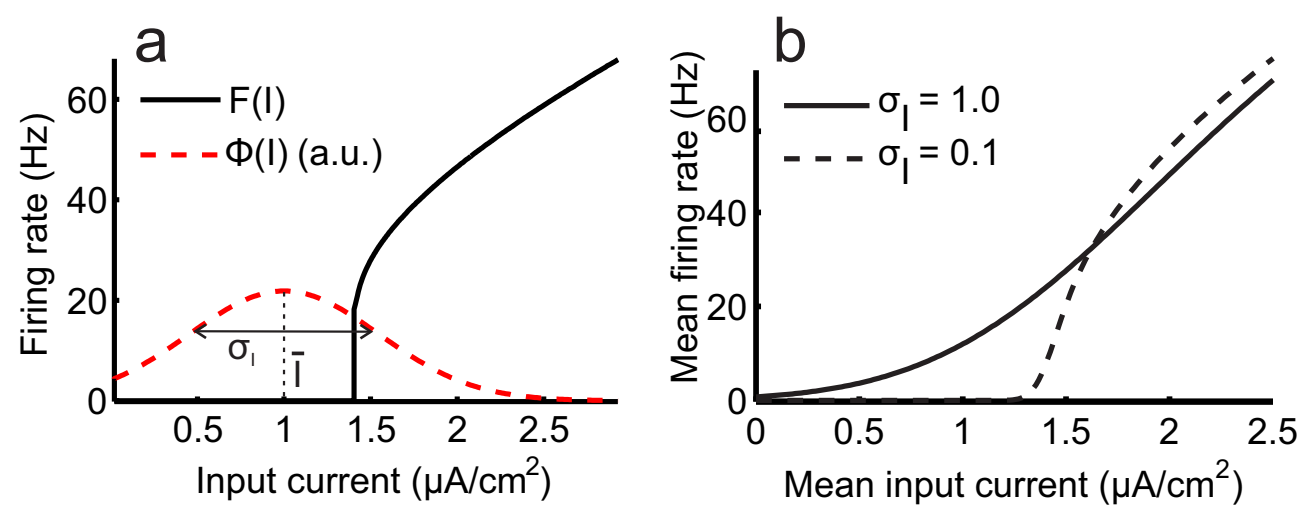

Figure 6.5: The functions involved in calculating the firing rate distribution. a) The distribution of the input currents and the $F(I)$-curve and $b$ ) the resulting sigmoidal function, depending on $\sigma_{I}$.

sum of contributions from many synapses (central limit theorem):

$$
I(t) \sim N\left(\bar{I}(t), \sigma_{I}^{2}(t)\right) .
$$

Hence, the mean $\bar{I}(t)$ and variance $\sigma_{I}^{2}(t)$ fully characterize the distribution function $\phi(I ; t)$ as:

$$
\phi_{y}(I ; t):=\phi\left(I ; \bar{I}_{y}(t), \sigma_{I_{y}}(t)\right)=\frac{1}{\sigma_{I_{y}}(t) \sqrt{2 \pi}} \exp \left(-\frac{1}{2}\left(\frac{\left(I-\bar{I}_{y}(t)\right)}{\sigma_{I_{y}}(t)}\right)^{2}\right) .
$$

Throughout this paper, the subscript $x$ denotes the presynaptic population or source $(\mathrm{e}, \mathrm{i}, \eta)$ and $y$ the postsynaptic population $(\mathrm{e}, \mathrm{i})$.

The mean $\bar{f}(t)$ and variance $\sigma_{f}^{2}(t)$ of the output firing rates can be calculated from the input current distribution and the F(I)-curve, as depicted in Fig. 6.5a:

$$
\begin{aligned}
\bar{f}_{y}(t) & =\int F_{y}(I) \phi\left(I ; \bar{I}_{y}(t), \sigma_{I_{y}}(t)\right) \mathrm{d} I \\
\sigma_{f_{y}}^{2}(t) & =\int\left(F_{y}(I)-\bar{f}_{y}(t)\right)^{2} \phi\left(I ; \bar{I}_{y}(t), \sigma_{I_{y}}(t)\right) \mathrm{d} I .
\end{aligned}
$$

In order for this to be valid, the $\mathrm{F}(\mathrm{I})$-curve must yield a good approximation for the instantaneous firing rate of neurons receiving fluctuating inputs. We will show in the Results that this is the case, provided that these fluctuations are on a time scale similar to or slower than the synaptic integration time. Note that the firing rates are not required to be normally distributed.

To obtain a sigmoid function in familiar form, equation 6.6 is equivalently described as the $\mathrm{F}(\mathrm{I})$-curve smoothed by convolution with a Gaussian of width $\sigma_{I}(t)$ :

$$
\bar{f}_{y}(t)=S_{y}\left(\bar{I}_{y}(t) ; \sigma_{I_{y}}^{2}(t)\right)
$$


The resulting sigmoid function $\mathrm{S}$ now depends on $\sigma_{I}(t)$, as shown in figure $6.5 \mathrm{~b}$.

We have now derived a sigmoid function based on the firing rate curve of the cells in the population, as was our main aim. The $\mathrm{F}(\mathrm{I})$-curve can be calculated numerically or analytically from the single cell model. The output firing rate distribution $\left(\bar{f}_{y}(t)\right.$ and $\left.\sigma_{f_{y}}^{2}\right)$ is a function of $\bar{I}(t)$ and $\sigma_{I}(t)$. These will be calculated from the input firing rates in the next sections.

\subsubsection{Distribution of the synaptic conductance}

Presynaptic activity causes the synapses to release neurotransmitter. This in turn opens ion channels on the postsynaptic membrane, increasing the conductance of these channels, which generates a synaptic current. The distribution of the input current will be calculated from the synaptic conductances.

We assume that cells in the network are randomly connected with synapses. These connections possibly have different strengths (weights). Cells receive synaptic connections from different populations and we refer to the group of all synapses from source $x$ to population $y$ as the synaptic population $x y$. Our model contains five synaptic populations, specifically ee, ie, $\eta$ e, ei, and ii (see figures 6.2 and 6.4). The conductances of the single synapses and their summation by the postsynaptic neurons are calculated in appendix 6.A. The derived expressions used in the neural mass model are summarized in this section.

The mean and variance of the conductance of the individual synapses $g_{x y}$ are obtained from the presynaptic firing rates $f_{x}$ (appendix 6.A):

$$
\begin{gathered}
\bar{g}_{x y}=H_{x y} * \bar{f}_{x}(t) \\
\sigma_{g_{x y}}=H_{x y} * \sigma_{f_{x}}(t) .
\end{gathered}
$$

Although the form of equation 6.10 is simple, the derivation is not trivial. In its derivation the presynaptic cells are assumed to fire periodically. In contrast, for the external input we assume Poisson (shot noise) statistics. Assuming all postsynaptic cells receive external input at the same rate $\lambda$, the mean and variance of the externally induced synaptic conductance are calculated as [13]:

$$
\begin{aligned}
\overline{G_{\eta}} & =H_{\eta e} * \lambda \\
\sigma_{G \eta}^{2} & =H_{\eta e}^{2} * \lambda .
\end{aligned}
$$

We define the total conductance $G_{x y}$ as the sum of the contributions of the individual synapses from source $x$ to a single neuron in population $y$. The distribution of $G_{x y}$ is determined (appendix 6.A) from the connection matrix. The mean $\bar{G}_{x y}$ is expressed as:

$$
\bar{G}_{x y}(t)=\bar{N}_{\mathrm{syn}, x y} H_{x y} * \bar{f}_{x}(t) .
$$


$N_{\text {syn }}$ is the mean (weighted) number of synaptic connections a neuron receives, $H$ the synaptic impulse response, i.e. PSP, of a single synapse and the asterisk denotes a convolution.

The variance $\sigma_{G}(t)$ is expressed as (appendix 6.A):

$$
\sigma_{G_{x} y}^{2}(t)=\operatorname{var}\left(N_{\mathrm{syn}, x y}\right) \bar{g}_{x y}(t)^{2}+N_{\mathrm{syn}, x y}^{\prime} \sigma_{g_{x} y}^{2}(t) .
$$

The first term is the variance due to differences in the number of synaptic connections received by the cells, the second term is the variance due to the variance in firing rates of the presynaptic neurons. $N_{\mathrm{syn}}^{\prime}$ is the expected (weighted) number of connections a postsynaptic neuron does not share with a random other cell of its population. This number is zero for an all-to-all connected network and approximately equal to $N_{\text {syn }}$ for a very sparsely connected network.

\subsubsection{Distribution of the input current}

With the expressions for the total synaptic conductances the distribution of the input current can be calculated. The total input current $I_{e}$ into a single cell in the excitatory population is calculated from the conductance of the three sources of input (see figure 6.4) as:

$$
I_{e}(t)=\left(E_{\mathrm{ampa}}-V_{\mathrm{th}}\right) G_{e e}(t)+\left(E_{\mathrm{gaba}}-V_{\mathrm{th}}\right) G_{i e}(t)+\left(E_{\mathrm{ampa}}-V_{\mathrm{th}}\right) G_{\eta e}(t)+\Delta I .
$$

The expressions for the inhibitory population are similar. $\Delta I$ is an effective synaptic input, added to simulate heterogeneity of the single cell parameters. $E_{\text {ampa/gaba }}$ is the reversal potential of the excitatory/inhibitory synapses, $G_{x y}$ the total synaptic conductance induced by population $x$ on a neuron in population $y$ and $\eta$ the conductance induced by external input. $V_{\text {th }}$ is the threshold potential of the neuron. Usually, the membrane voltage itself, rather than the threshold voltage, is used to calculate the input current. However, a spiking neuron spends most time close to threshold. Hence, this yields a good approximation to determine the spike rate. Subthreshold input currents are calculated incorrectly, but this is inconsequential, since the spike rate will be determined correctly as zero.

Assuming the distribution of the total conductances from the different sources are independent, the mean and variance of the input current are calculated as:

$$
\begin{aligned}
\bar{I}_{e}(t) & =\left(E_{\mathrm{ampa}}-V_{\mathrm{th}}\right) \bar{G}_{e e}(t)+\left(E_{\mathrm{gaba}}-V_{\mathrm{th}}\right) \bar{G}_{i e}(t)+\left(E_{\mathrm{ampa}}-V_{\mathrm{th}}\right) \bar{G}_{\eta e}(t), \\
\sigma_{I e}^{2}(t) & =\left(E_{\mathrm{ampa}}-V_{\mathrm{th}}\right)^{2} \sigma_{G_{e e}}^{2}(t)+\left(E_{\mathrm{gaba}}-V_{\mathrm{th}}\right)^{2} \sigma_{G_{i e}}^{2}(t)+\left(E_{\mathrm{ampa}}-V_{\mathrm{th}}\right)^{2} \sigma_{G_{\eta e}}^{2}(t)+\sigma_{\Delta I}^{2} .
\end{aligned}
$$

These expressions close our set of equations, which can now in principle be analyzed and simulated. First however, more computationally efficient expressions for the convolutions (Eqs. 6.10-6.12) will be given in the next section. 


\subsubsection{Differential equations for the synaptic dynamics}

For computational efficiency as well as analytical tractability, a synaptic impulse response $H$ is chosen such that the convolutions can be described with ordinary differential equations. Common choices are the exponential and the alpha synapse, with impulse responses (PSP's) of

$$
H_{\mathrm{exp}}=g_{0} e^{-t / \tau}
$$

and

$$
H_{\alpha}=e g_{0} \frac{t}{\tau} e^{-t / \tau},
$$

respectively. Here $g_{0}$ is the peak amplitude of the PSP and $\tau$ the synaptic time constant. The corresponding differential equations are:

$$
\frac{\mathrm{d} g}{\mathrm{~d} t}=-\frac{g}{\tau}+g_{0} f(t)
$$

and

$$
\frac{\mathrm{d}^{2} g}{\mathrm{~d} t^{2}}=-\frac{2}{\tau} \frac{\mathrm{d} g}{\mathrm{~d} t}-\frac{g}{\tau^{2}}+\frac{e}{\tau} g_{0} f(t) .
$$

The convolution with $H^{2}$ (Eq. 6.12) can be written as:

$$
\frac{\mathrm{d} \sigma^{2}}{\mathrm{~d} t}+2 \frac{\sigma^{2}}{\tau}=g_{0}^{2} f(t)
$$

and

$$
\frac{\mathrm{d}^{3} \sigma^{2}}{\mathrm{~d} t^{3}}+\frac{6}{\tau} \frac{\mathrm{d}^{2} \sigma^{2}}{\mathrm{~d} t^{2}}+\frac{12}{\tau^{2}} \frac{\mathrm{d} \sigma^{2}}{\mathrm{~d} t}+\frac{8}{\tau^{3}} \sigma^{2}=2 \frac{e^{2} g_{0}^{2}}{\tau^{2}} \lambda(t),
$$

for the exponential and alpha synapse respectively. ${ }^{\dagger}$

We used the alpha synapses in our implementation. However, to reduce the dimensionality and improve computational efficiency when calculating the variances, $H$ and $H^{2}$ were approximated with exponential functions. To obtain equal steady state values and approximately equal rise times, we chose approximations that have equal areas, and equal second moments around $t=0$. This gives values of $g_{0}=e / 2 g_{0, \alpha}$ and $\tau=2 \tau_{\alpha}$ for $H$ and $g_{0}=e / \sqrt{(6)} g_{\alpha}$ and $\tau=3 \tau_{\alpha}$ for $H^{2}$.

\subsection{Implementation and simulations}

First, we describe the implementation of a network of spiking neurons, used as a standard for validation, and then the implementation of our new neural mass model.

\footnotetext{
${ }^{\dagger}$ I would like to thank Hil Meijer for presenting me with the latter equation.
} 


\subsubsection{Network of spiking neurons}

The Norns - Neural Network Studio was used to build and simulate the network of spiking cells. Norns is designed for easy analysis of networks of spiking neuron models ${ }^{\ddagger}$.

The single cells were modeled with a Hodgkin-Huxley (HH) model with voltage gated $\mathrm{Na}^{+}$- and $\mathrm{K}^{+}$-channels and leak currents (Eqs. 1-3 of [14]). The cells were synaptically connected with alpha synapses. These were chosen since they are a second order system, which has been shown to generate more realistic frequency spectra than exponential synapses [10]. Differential equations for the HH model and the alpha synapse (equation 6.21) were added as new cell and synapse types to the Norns package. An action potential (spike) was defined as the membrane voltage crossing $0 \mathrm{mV}$ from below.

A network was built consisting of two populations of 100 cells each (see figure 6.2). For simplicity, the same parameters were chosen for all cells. Then, heterogeneity of the population was obtained with an additional sodium leak conductance, normally distributed over the cells. This conveniently allows us to model this heterogeneity as an extra synaptic input.

Synaptic currents were included next to the leak and gated currents. They are expressed as usual as $I=g_{\text {syn }}\left(E_{\text {syn }}-V\right)$, where $V$ is the neuron's membrane voltage. Synaptic connections, represented in connection matrices $W_{x y}$, were randomly made between the cells with a probability $p$, all having equal strength $(w=1)$. Different strengths of the synaptic populations $(\mathrm{e} \rightarrow \mathrm{e}, \mathrm{e} \rightarrow \mathrm{i}$, etc.) were obtained by choosing different PSP amplitudes $g_{0}$.

The excitatory cells were given external input via excitatory synapses, that received Poisson distributed spikes with rate $\lambda$. To show how the populations react to a change in input, a double step in input was given:

$$
\lambda= \begin{cases}0 \mathrm{~s}^{-1} & t<0 \mathrm{~s} \\ 600 \mathrm{~s}^{-1} & 0 \mathrm{~s}<t<0.5 \mathrm{~s} \\ 400 \mathrm{~s}^{-1} & 0.5 \mathrm{~s}<t\end{cases}
$$

The synaptic currents that are induced by this input are depicted in figure 6.6.

\subsubsection{The new neural mass model}

First, the F(I)-curve was calculated by simulating a single $\mathrm{HH}$ cell, as described in the previous section, for a range of constant conductivities of the excitatory synapses in the simulation. F was determined from the interspike interval (ISI). The reported $I$ is calculated as $I=g\left(E_{\mathrm{ampa}}-V_{\mathrm{th}}\right)$. Alternatively, a current could have been injected

\footnotetext{
$\ddagger$ available from: http://wwwhome.math.utwente.nl/ visser/
} 


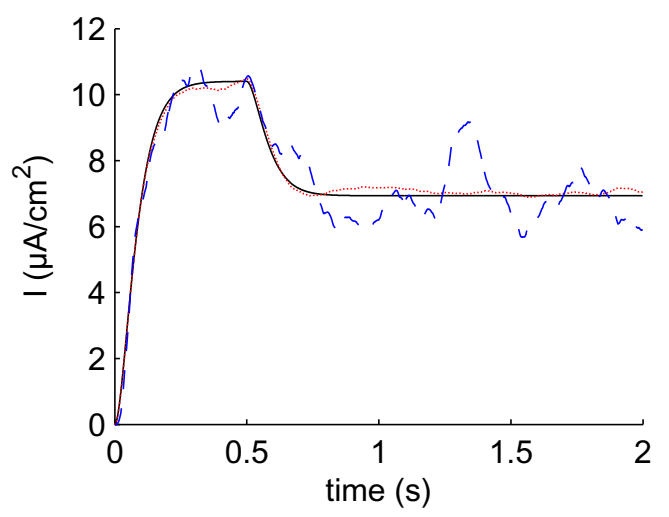

Figure 6.6: Synaptic currents induced by (Poisson distributed) external input in our simulation. Depicted are the expected current (solid line), and a typical realization for a single synapse (dashed line) and the mean of 100 synapses (dotted line).

directly into the cells. The former method leads to more accurate results, since it takes the shunting effect of the excitatory synaptic conductance into account. For simplicity, the neurons in the two populations were given the same parameter values and hence have the same $\mathrm{F}(\mathrm{I})$-curve. The threshold voltage of the $\mathrm{HH}$ model was determined numerically, typically $-55 \mathrm{mV}$.

The values for the parameters representing the number of synapses were calculated from the generated connection matrices $W_{x y}$ used in the network model. For a binomial distribution with $p=0.5$ and $N=100$, this generates on average values of $\bar{N}_{\text {syn }}=50, \operatorname{var}\left(N_{\text {syn }}\right)=25$ and $N^{\prime}=25$ for all synaptic populations, i.e. the cells receive $50 \pm \sqrt{25}$ synapses of each type, and two cells share on average 50-25 synaptic connections of each type. Note that $\operatorname{var}(N)=N_{\text {syn }}^{\prime}$ is a property of the binomial distribution.

The differential equations of the neural mass model (see method section) were implemented in Matlab. The differential equations were solved with a forward Euler time stepping algorithm, using a time step of $0.1 \mathrm{~ms}$. The second order differential equation for the alpha synapse was used to calculate $\bar{g}$ for each of the five synaptic populations. To reduce the dimensionality, $\sigma_{g}$ was calculated using an exponential approximation (see section 6.2.4). Because the variance of $g$ has only a secondary effect on the dynamics, the errors on the firing rates and synaptic conductances introduced by this approximation are small.

A noisy input signal for the NMM was generated by binning the random external input spike times of the simulation of the network model ( $\Delta t=1 \mathrm{~ms})$, allowing to compare the two models with the same input. 


\subsubsection{Parameters}

For the single $\mathrm{HH}$ cells, conductances and time constants were chosen as in [14]. Nernst potentials were chosen in the physiological range as $E_{k}=-95 \mathrm{mV}, E_{\mathrm{na}}=53$ $\mathrm{mV}, E_{\mathrm{cl}}=-82 \mathrm{mV}$. We simulated 4 different networks. Our standard parameter set defines a network of type 1 spiking neurons in which there is relatively weak feedback between the populations. We further tested our approach by increasing the feedback, i.e. the strength of the $i \rightarrow e$ synapses, and by simulating a network with type 2 spiking cells. To demonstrate changes in single cell dynamics can easily be incorporated, an increased extracellular potassium concentration is modeled. In summary, four cases are modeled:

1. a network of type 1 spiking neurons

2. the same network with strong feedback between the populations ( $g_{0 \eta}=5.4$ and $g_{0 i e}=8 \mu \mathrm{S} / \mathrm{cm}^{2}$ )

3. a network of type 2 spiking neurons

4. network 1 with increased $E_{k}(-80 \mathrm{mV})$

A type 1 neuron was created from the standard $\mathrm{HH}$ parameters by increasing the membrane capacitance $C_{m}$ to $10 \mu \mathrm{F} / \mathrm{cm}^{2}$, while the original value of $C_{m}=1.0 \mu \mathrm{F} / \mathrm{cm}^{2}$ was used to model type 2 spiking behavior. Heterogeneity of the cells was modeled by an extra sodium conductance that was normally distributed, with a width corresponding to an input current of $\sigma_{\Delta I}=0.5 \mu \mathrm{A} / \mathrm{cm}^{2}$, approximately a third of the threshold current. Time constants were chosen based on the membrane time constants obtained in [10]. These were halved, since the alpha function is wider than an exponential function, in order to obtain similar synaptic integration times. Table 6.1 shows the synaptic parameters. Values for the synaptic strengths were chosen such that the steady state firing rates of both populations were approximately equal in the range between 4 and $100 \mathrm{~Hz}$. A mean firing rate was obtained around approximately $30 \mathrm{~Hz}$ by tuning the strength of the external input.

Table 6.1: Synaptic parameters

\begin{tabular}{lllccccc} 
Synaptic population & & & $\eta \rightarrow \mathrm{e}$ & $\mathrm{e} \rightarrow \mathrm{e}$ & $\mathrm{e} \rightarrow \mathrm{i}$ & $\mathrm{i} \rightarrow \mathrm{e}$ & $\mathrm{i} \rightarrow \mathrm{i}$ \\
\hline Connection probability & $p$ & & & 0.5 & 0.5 & 0.5 & 0.5 \\
Time constant & $\tau$ & $(\mathrm{ms})$ & 45 & 45 & 34 & 45 & 34 \\
Reversal potential & $E_{\mathrm{syn}}$ & $(\mathrm{mV})$ & 50 & 50 & 50 & -82 & -82 \\
Synaptic strengths & & & & & & & \\
Network w. type 1 cells & $g_{0}$ & $\left(\mu \mathrm{S} / \mathrm{cm}^{2}\right)$ & 1.4 & 0.06 & 0.45 & 0.11 & 0.15 \\
Network w. type 2 cells & $g_{0}$ & $\left(\mu \mathrm{S} / \mathrm{cm}^{2}\right)$ & 0.8 & 0.06 & 0.45 & 0.8 & 1.5
\end{tabular}




\subsection{Data analysis of the network simulations}

Spike times and synaptic conductances for each cell were recorded from the network model. From the synaptic conductances, synaptic currents were calculated from the terms in equations 6.16 and 6.17. For some simulations the frequency spectrum of the synaptic current was obtained with the fast Fourier transform (FFT) of an 8 second long segment with constant input rate. The mean firing rate in the network model was determined for both populations by binning the spikes in intervals of $5 \mathrm{~ms}$. The standard deviation of the firing rates were calculated from the instantaneous frequencies of the individual cells, determined by their interspike intervals. This simple method yields artifacts when the population spike rate changes rapidly. Sections with artifacts were bleached in the figures shown in the Results.

One of the main assumptions in the derivation of our NMM was that the firing rate of the cells in the network is determined by their instantaneous input current. To test whether this approximation was accurate, for each cell in the network the spike rate was calculated both from the recorded interspike intervals as well as from the recorded synaptic conductances, according to equation 6.15 .

Besides the spike timings of the external input, none of the recorded quantities were given to the NMM.

\subsection{Results}

We show simulation results for several parameter values, that allow us to illustrate our main conclusions on the constructed NMM. To validate our model, we compare the new NMM with simulations of a network of type 1 spiking neurons, with both weak and strong feedback between the populations, and with a network of type 2 neurons. We then show how our neural mass model can be used to model changes in single cell parameters. As an example, an increased extracellular potassium concentration was modeled. The F(I)-curves for these three different neurons were calculated, and are shown in figure 6.7. After validating the new NMM with the spiking network, we verify that the firing rates of the single cells in the network indeed instantaneously depend on their synaptic input, as was assumed in the derivation of the NMM.

\subsubsection{Type 1 neurons}

First we show and discuss the results for a network consisting of two populations of 100 type 1 cells, with relatively weak feedback., i.e. the dynamics of the excitatory population are mainly determined by the external input and the dynamics of the inhibitory population are mainly determined by the firing rate of the excitatory cells. Figure 6.8 shows that the NMM model describes the dynamics of the network of neurons very well, both for the synaptic conductances (mean and variance) and the firing 


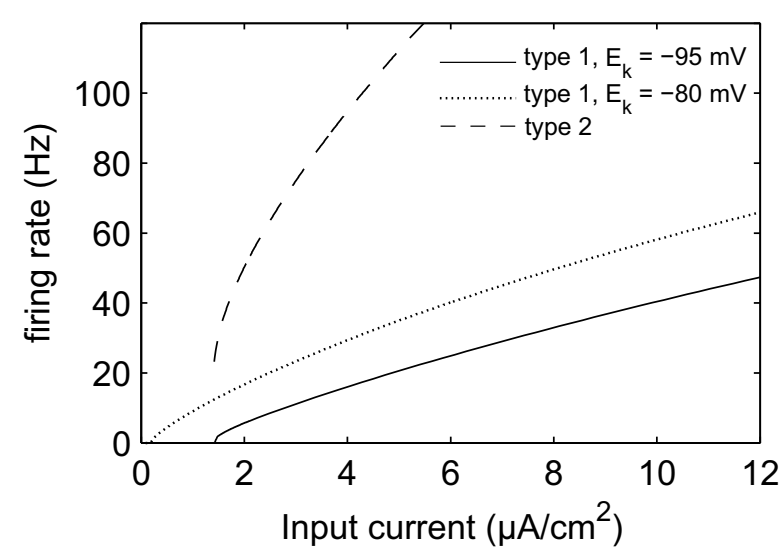

Figure 6.7: $F(I)$-curves of the single cells. Shown are the responses of a type 1 spiking neuron (full line), the same neuron with an increased extracellular potassium concentration, and a type 2 spiking neuron (dashed line.) The curves were obtained from simulations of single cells (HH-model) with a range of excitatory input conductances.

rates (mean and variance). The fluctuations in the standard deviations are caused by the small size of the modeled network. These are stochastic in nature and hence are not reproduced by the NMM.

Figures 6.9 and 6.10 show a close-up of the dynamics of one of the synaptic currents and its frequency spectrum. The new NMM reproduces the low frequency part $(<20 \mathrm{~Hz})$ of the firing rate signal very accurately.

The synaptic currents in the spiking network model show small oscillations in the frequency band around the mean firing rates of the populations. These oscillations are caused by some of the neurons firing in phase. As expected, the NMM does not produce these oscillations, since neural mass models assume that phase effects are averaged out.

\subsubsection{Stronger feedback}

In the previous simulations, the feedback from the inhibitory to the excitatory population was relatively weak. We further test our newly proposed NMM by simulating a network with strong feedback. Increasing the strength of the $i \rightarrow e$ synapses leads first to the appearance of a resonance peak in the spectrum, i.e. spindles in the time domain. This is followed by the appearance of a limit cycle. Figure 6.11 shows the dynamics of network 2 , where $g_{0, i, e}$ has been increased from 0.1 to $8 \mu \mathrm{S} / \mathrm{cm}^{2}$. The input strength $g_{0, \eta}$ is increased from 1.4 to $5.4 \mu \mathrm{S} / \mathrm{cm}^{2}$ to compensate for the inhibition. The NMM underestimates the amplitude of the oscillations, also in the resonating regime (not shown) and the time courses of the synaptic currents are more sinusoidal than those in the network model. However, the approximate frequency of 

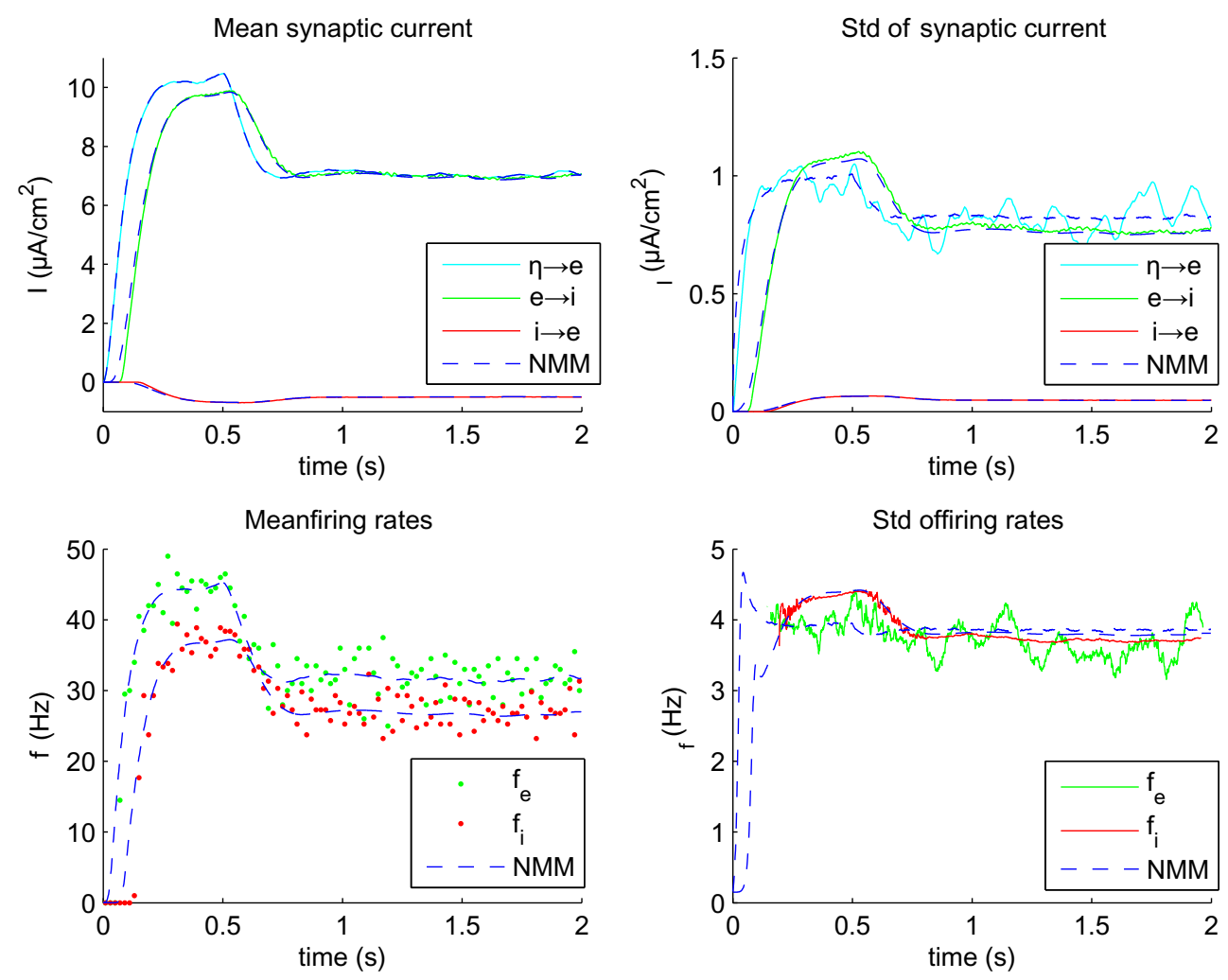

Figure 6.8: Comparison of our new NMM with a detailed network model. The reaction to a double step in input rate of the network model (full lines and dots) is compared to that of the NMM (blue dashed lines). The mean and standard deviation of three synaptic currents are displayed, as well as those of the firing rates of the excitatory and inhibitory populations. 


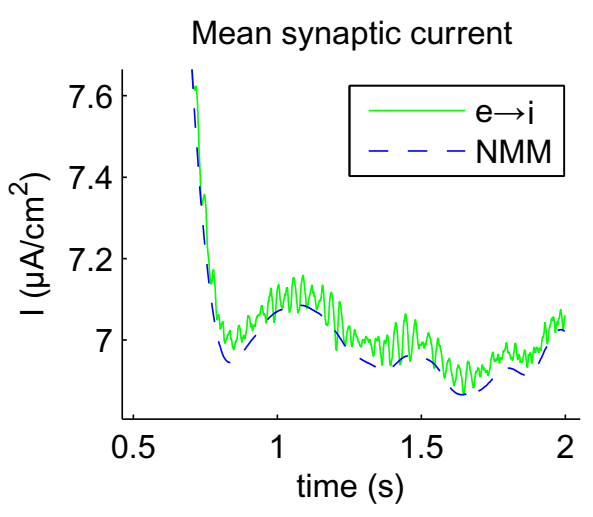

Figure 6.9: Close up of the mean synaptic current (figure 6.8, upper left panel). The new NMM predicts the DC value accurately within $1 \%$ error. Also, the low frequency oscillations observed in the network model are accurately reproduced.

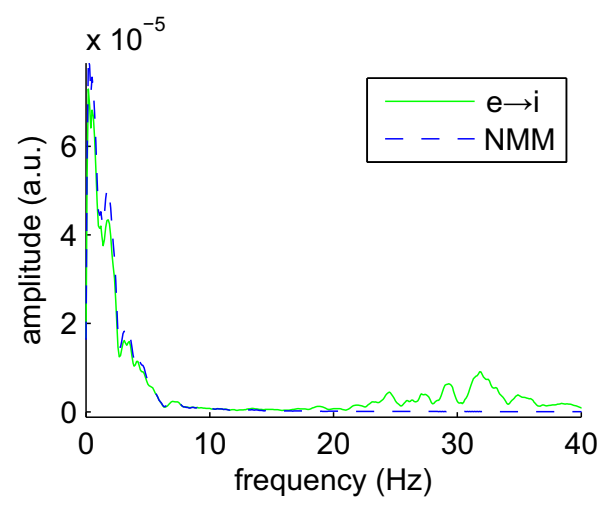

Figure 6.10: Frequency spectrum of the synaptic current. The frequency spectrum is accurately reproduced below $18 \mathrm{~Hz}$. A broad peak is observed around $30 \mathrm{~Hz}$, the mean firing rate, in the dynamics of the network model. As expected, this is not reproduced by the new NMM.

the limit cycle, as well as the relative phases and amplitudes of the firing rates and synaptic currents of the two populations are well reproduced.

\subsubsection{Type 2 neurons}

The F(I)-curve of the type 1 neurons is almost straight for frequencies above $10 \mathrm{~Hz}$. Hence the sigmoidal function (equation 6.8) is almost equal to the F(I)-curve regardless of the variance in the input current, and the variance does hardly influence the dynamics of the synaptic conductances. Therefore, we test whether our new NMM can also reproduce the behavior of a network with two populations consisting of type 2 firing neurons. In this network, the width of the input current distribution has a larger influence on the dynamics, due to the step in the F(I)-curve. Advantageous for our approach is that the single cell dynamics have a negligibly small region of bistability.

Again our new NMM is compared to a network of spiking cells in figure 6.12. The oscillations of the synaptic currents and firing rates in the new NMM are less pronounced than in the spiking network, but the general behavior is very well reproduced. The mean of the firing rate is slightly underestimated, what in turn causes the standard deviation of the synaptic currents and firing rates to be underestimated. Considering that the network operates close to the thresholds of the neurons, where the standard deviation of the firing rates is very sensitive to the synaptic current distribution, the new NMM performs well. 

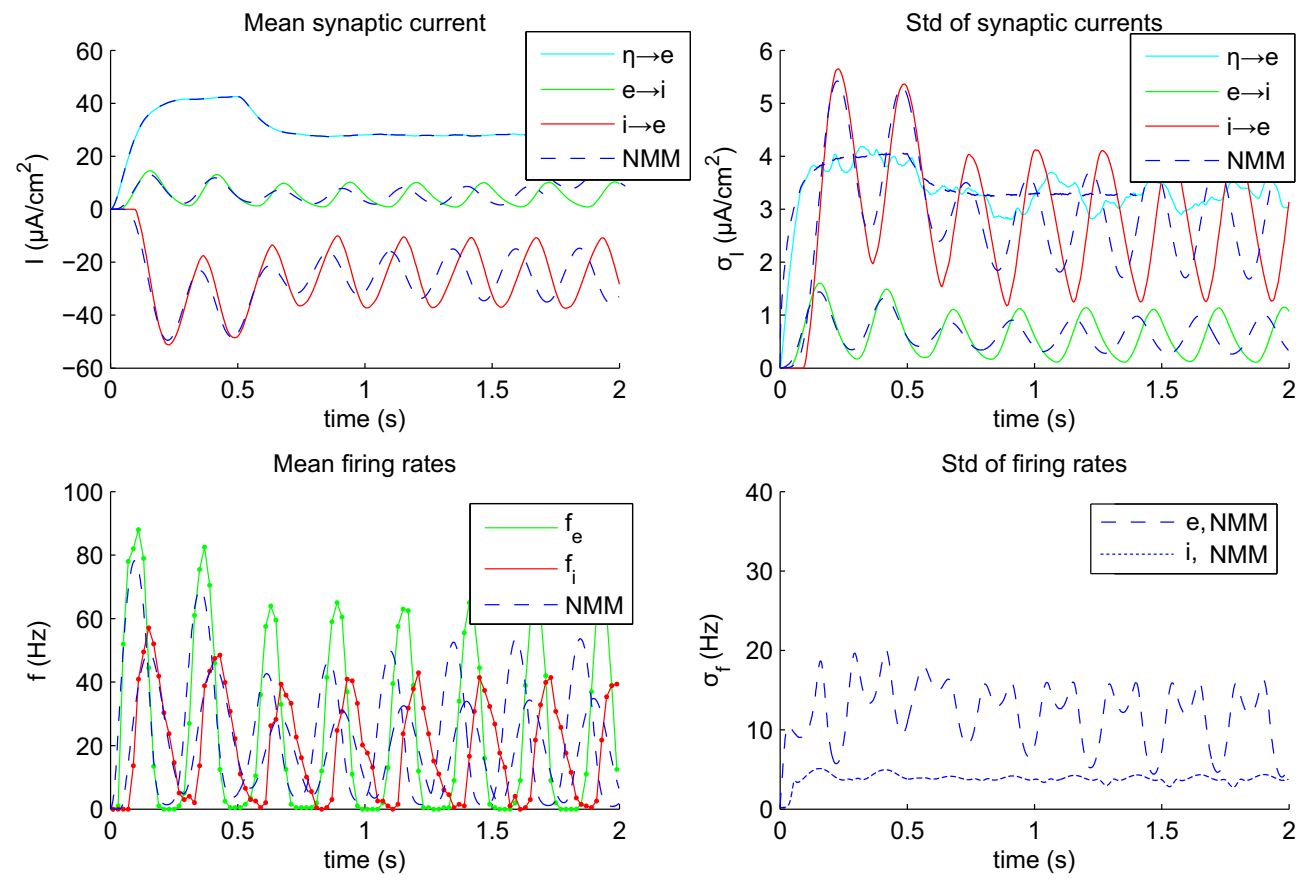

Figure 6.11: Simulations of a network with strong feedback. The reaction to a double step in input rate of the new NMM (blue dashed lines) is compared to that of the network model (full lines and dots). The mean and standard deviation of three synaptic currents are displayed, as well as the firing rates of the excitatory and inhibitory populations. The standard deviations of the firing rates of the network model are not shown since they are dominated by artifacts. 

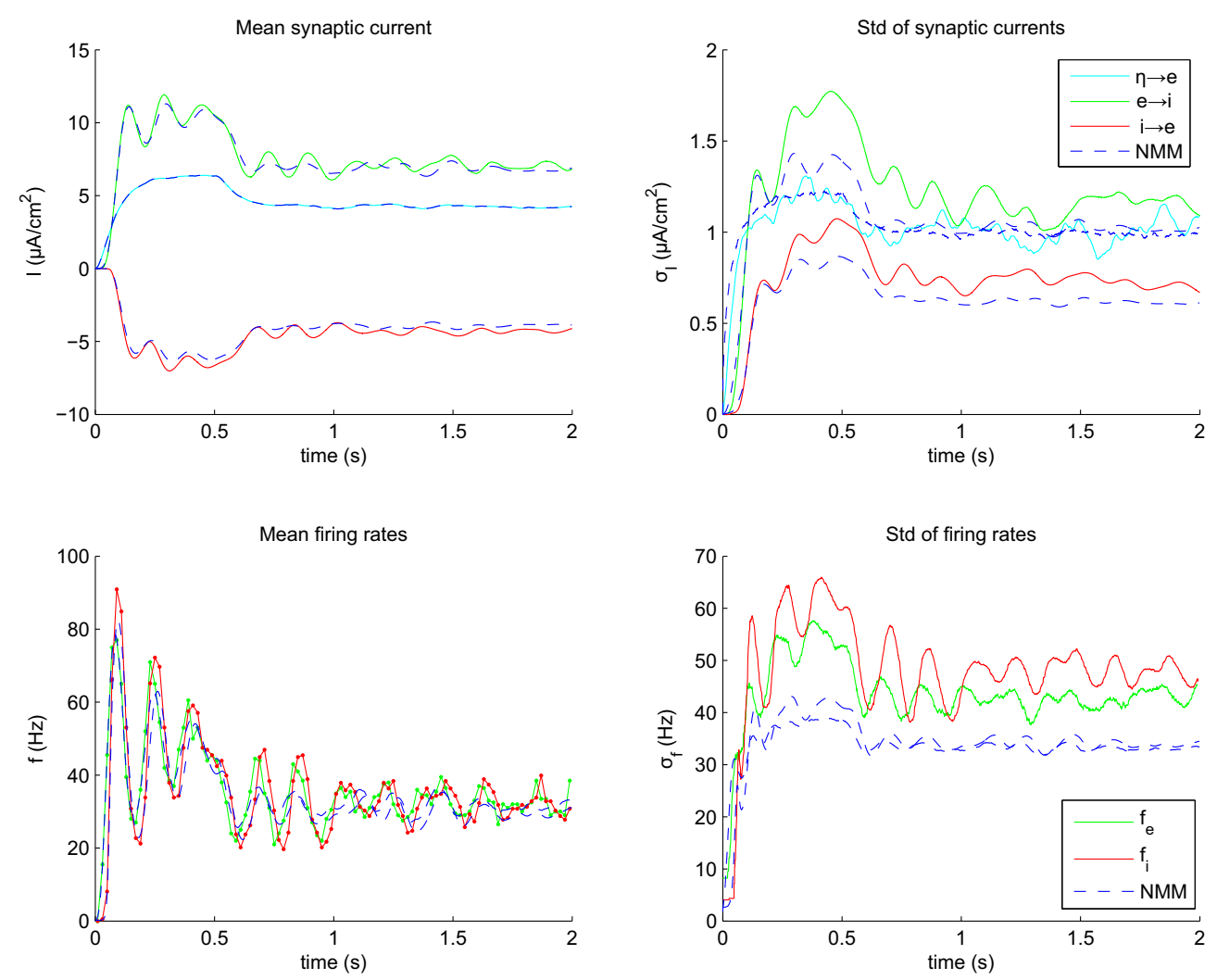

Figure 6.12: The dynamics of two populations of type 2 spiking neurons. The reaction to a double step in input rate of the NMM (blue dashed lines) is compared to that of the network model (full lines). The mean and standard deviation of three synaptic currents are displayed, as well as those of the firing rates of the excitatory and inhibitory populations. 

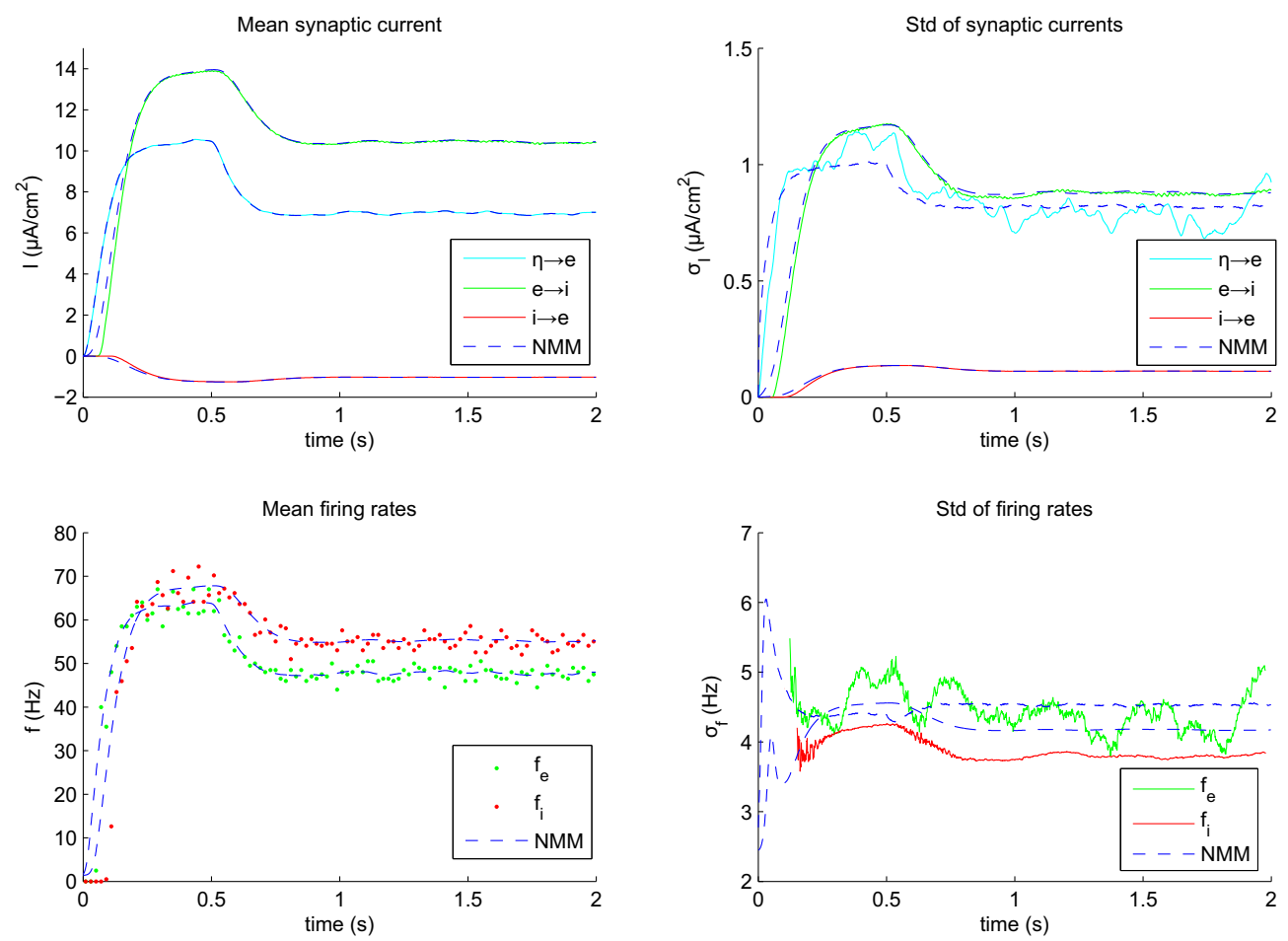

Figure 6.13: Simulations of increased extracellular potassium. The reaction to a double step in input rate of the new NMM (blue dashed lines) is compared to that of a spiking network model (full lines and dots). The mean and standard deviation of three synaptic currents are displayed, as well as those of the firing rates of the excitatory and inhibitory populations. The increased extracellular potassium causes the firing rates of both populations to increase.

\subsubsection{Increased extracellular potassium}

To demonstrate that our approach can be used to easily model the effects of changes in single cell dynamics, the potassium Nernst potential was increased to $-80 \mathrm{mV}$ in the spiking network model. This typically corresponds to an increase of the extracellular potassium concentration from 4 to $7 \mathrm{mM}$. This increases the excitability of the neurons. To adapt the neural mass model, a new F(I)-curve was calculated, as described in section 6.3.2 (see figure 6.7). The increased potassium concentration shifts the firing rate curve to the left. A new threshold voltage was determined as -56 $\mathrm{mV}$.

The results of the simulations are shown in figure 6.13. The new NMM predicts that the increase in extracellular potassium increases the firing rates of both populations, without further remarkable changes in the dynamics (c.f. figure 6.8). The shifts in firing rates predicted by the neural mass model accurately correspond to 

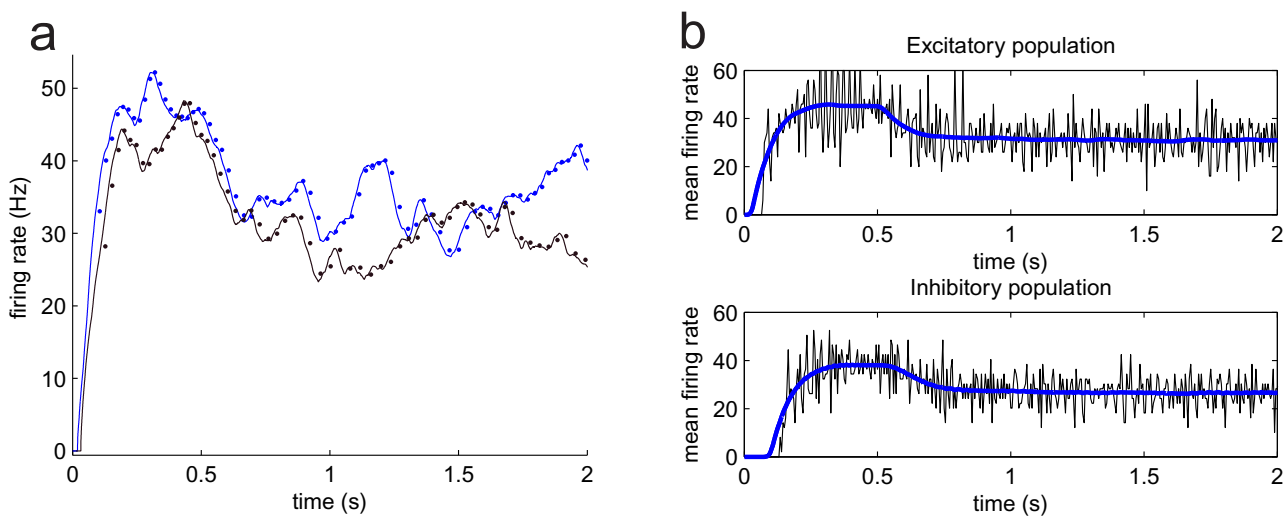

Figure 6.14: Firing rates assumed from the synaptic input versus observed firing rates. Panel a shows the firing rates of two randomly chosen excitatory cells during the simulation shown in figure 6.8. The full lines represent the firing rates calculated from the $F(I)$-curve and $I(t)$ of the individual neurons. The dots are the spike rates determined from the recorded interspike interval. Panel $b$ shows the average for all neurons determined from the binned spike times (thin black line) and the rate calculated from the input currents (thick blue line).

those observed in the network of spiking neurons.

\subsubsection{Relation of input and firing rate of the single cells}

The actual firing rates observed in the spiking network model are compared to those predicted from the synaptic input the individual cells receive in figure 6.14. Panel a compares these for two randomly chosen (excitatory) cells. The firing rate is predicted accurately from the instantaneous synaptic conductances, even though the synaptic input fluctuates on a time scale similar to the firing rate. Panel $b$ shows the two rates for the entire population. Even though the synaptic input predicts the firing rate for the single cells accurately, the firing rate of the population shows fluctuations around the predicted curve. This shows that the firing of the cells is to some extent synchronized.

Even more pronounced synchronization is found during large amplitude limit cycles, shown in figure 6.15. The firing rates of the populations in the network model exhibit a damped oscillation around the rates predicted from the input currents, especially clear for the inhibitory population.

\subsection{Discussion}

Neural mass models are designed for modeling macroscopic electrical activity of the brain, which is reflected in the EEG. Pathologies or medication, such as chan- 

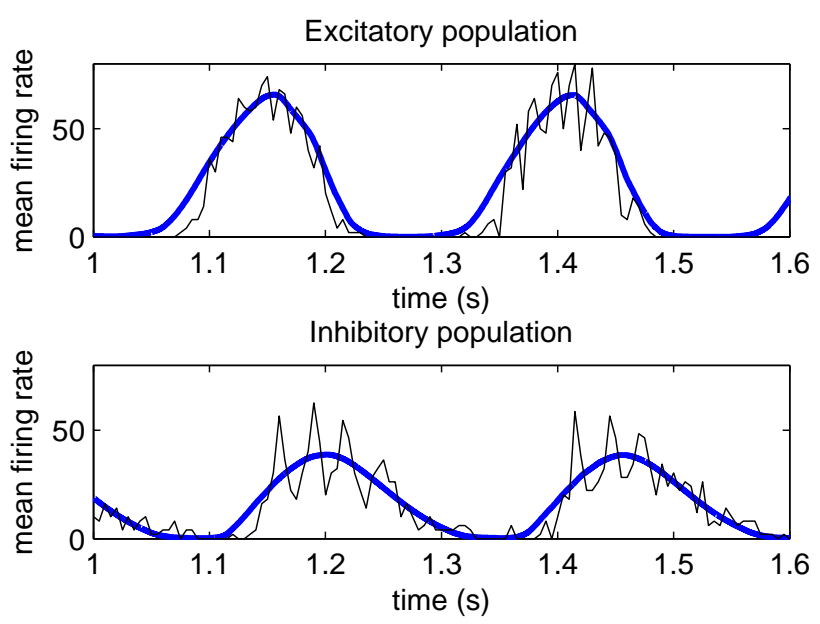

Figure 6.15: Population firing rate during a limit cycle. The firing rate assumed in deriving the NMM was determined from the individual input currents (thick blue line), the observed rate in the spiking network (thin black line) was determined from spike binning. A delayed response of the population to the synaptic current can be observed, followed by a damped oscillation around the curve predicted from the synaptic current. This is especially clear in the inhibitory population.

nel blockers, hypoxia or anesthesia, can alter this activity. In existing neural mass models, the synaptic response is explicitly modeled, which allows for investigating neural dynamics during altered synaptic functioning. To also include the electrophysiology of the single cell dynamics explicitly, we have constructed a new neural mass model, with a sigmoid function derived from the single cell $\mathrm{F}(\mathrm{I})$-curve. This new model was validated by quantitatively comparing it with a network of spiking neurons, representing neural tissue with pyramidal cells and interneurons. This spiking network model consisted of both excitatory and inhibitory Hodgkin-Huxley neurons. The newly derived neural mass model was shown to describe the synaptic activations and the population firing rates accurately.

Using the F(I)-curve as link between single cell and macroscopic dynamics has several distinct advantages. It can easily be calculated numerically from a physiological plausible model, without reduction or analysis of the dynamics. The curve is a familiar function for most and the influence of pathologies on its shape can be understood intuitively. It carries information on excitability, sensitivity and maximum firing rates. Furthermore, depolarization block of the cells, important for example during certain types of epilepsy [15], is naturally included in the F(I)-curve.

The usual techniques can be used to analyze the dynamics of the model for changes in the F(I)-curve. For example, the dependency of the steady state firing 
rates on $E_{K}$ can be calculated from an analysis of the nullclines of the system, and linearizing the NMM around a fixed point and calculating the transfer function between the input and the synaptic conductance yields the power spectrum.

The approach presented for constructing the sigmoidal function in our NMM can be used to adapt existing neural mass models as well, for example the Jansen en Rit model [16] or the Robinson model [17]. This allows to investigate the effects of altered single cell dynamics on the EEG signals predicted by these models.

There are, however, some restrictions on our approach, which we will now discuss. Then we discuss possibilities for including in the model some effects that were neglected, such as unreliability of the synapses. Finally we will deliberate on similar investigations by others, and how these complement this work.

\subsubsection{Valid parameter ranges}

The main assumption for neural mass modeling in general is that a neuron's input current consists of contributions from many action potentials, i.e. $N_{\text {syn,xy }} f_{x} \tau_{x y} \gg 1$. This is the case for typical physiological parameters in cortex, e.g. $N=2000, f=4$ $\mathrm{Hz}$ and $\tau=50 \mathrm{~ms}$, for which a cell receives 400 action potentials during a synaptic integration time.

A second assumption made in our derivation is that the synaptic input currents instantaneously determine the firing rate. We have shown this is the case when the synapses fluctuate on a time scale slower than or similar to the spike rate, i.e. $\tau_{x y} \gtrsim 1 / f_{y}$. Hence, dynamics involving low spike rates or very short somatic PSP's are not modeled accurately. By assuming the $\mathrm{F}(\mathrm{I})$-curve characterizes the instantaneous firing rate, action potentials resulting from subthreshold fluctuations were neglected. Further work is needed to find whether low firing rates can be modeled by incorporating the finite firing rates for subthreshold input into the $F(\mathrm{I})$-curve. This would be necessary depending on which brain area is modeled. While cortical neurons typically have low average firing rates, rates are higher in other brain structures.

To test our model, we have chosen synaptic time constants of 45 and $34 \mathrm{~ms}$ for the response by excitatory and inhibitory cells respectively. This number may seem high when compared with the synaptic time constants used by e.g. Liley et al. ( 3 and $15 \mathrm{~s}$ for excitatory and inhibitory synapses, respectively). However, Liley et al. filter the synaptic responses with an additional linear equation representing the membrane, for which they obtain time constants of 90 and $65 \mathrm{~ms}$ to reproduce experimental EEG's. We have based our choice of the time constants on these numbers to obtain similar synaptic integration times. Alternatively, we could have used a ball-and-stick single neuron model rather than a point model. In that case, a faster time constant could have been chosen for the synaptic response, that is subsequently filtered with a slower response of the dendrite. 


\subsubsection{Phase synchronization}

An assumption in neural mass modeling is that the phases of the individual cells cancel out. However, three different mechanisms cause synchronization.

The first mechanism, exemplified in figure 6.15, is caused by a sudden increase in the input current of a population [1]. A population density approach using the timesince-last-spike can accurately model this phenomenon for current based models. Furthermore, the output lags, for sudden changes in input current [18]. The approach of Ostojic and Brunel using a frequency dependent lag [19], can be used to investigate this. This lag and synchronization are transient phenomena, and therefore not of interest when predicting the dynamics of ongoing neural activity.

The second mechanism is that the phases of a finite amount of neurons never completely cancel. However, the relative amplitude of the oscillations this induces in the synaptic current, declines as $1 / \sqrt{N}$. This is negligible for a large number of cells $N$ in a population. Therefore, our NMM actually yields a better description of the activity of large population of neurons than the (small) network model used for validation.

A third mechanism is the tendency of neurons to synchronize with modulations in their input. A population's firing rate hence amplifies modulations in the input at frequencies similar to the modal firing rate. Shriki et al. have shown this effect can be modeled by filtering the input current with a (phenomenological) second order resonance filter before calculating the firing rate [20]. This amplification is expected to be larger for two coupled populations than for the single population described by them. The tendency to synchronize also occurs in our simulations of the network model. We did not investigate how this synchronization scales with $N_{\text {syn }}$ and $\operatorname{var}\left(N_{\text {syn }}\right)$, because it was not possible in our implementation to uncouple the relevant parameters for this mechanism (number of cells and synaptic connections, fluctuations in external input and width of synaptic current distributions).

In summary, our neural mass model does per definition not show any phase synchronization effects. These have been shown to occur, also in large populations of neurons, and result in oscillations at frequencies near the modal firing rates. This should be kept in mind when interpreting the dynamics of any neural mass model.

\subsubsection{Modeling additional effects}

The inhomogeneity of the single cell parameters was chosen such that it could be described as an additional input current, i.e. a shift in the $\mathrm{F}(\mathrm{I})$-curve for each neuron. In the case of heterogeneous properties of the neurons that do more than shift the $\mathrm{F}(\mathrm{I})$ curve, two curves can be determined from the single cell description. The average $\mathrm{F}(\mathrm{I})$-curve of the population, as well as the $\sigma_{\mathrm{fp}}^{2}(\mathrm{I})$-curve, the variance of the firing rate over the population for the same I. This term can be added to variance of the firing 
rate (equation 6.6).

We have assumed reliable synapses, that release a quantum of neurotransmitter with every action potential. Actual synapses are known to be quite unreliable, and typically fail to activate $>50 \%$ upon receiving an action potential [21]. We did not investigate unreliability, but this can be included in the model as an additional term in the variance.

Furthermore, we have assumed that the inhibitory conductance can effectively be subtracted from the excitatory conductance (equation 6.16). However, when the inhibitory synaptic current is large compared to the net input current, we found (not shown) that it also shunts the gated currents. This reduces the spike rate. To model this, equation 6.16 could be adapted with an extra non-linear term.

Finally, we assumed the neurons in our population have a simple F(I)-curve, and do not exhibit, for example, bistability or bursting. Mathematical techniques have been investigated to include bursting into neural mass models [22, 23]. These are also applicable to our approach.

\subsubsection{Complementary work}

When our NMM is operated in a regime where the F(I)-curves are relatively straight, the variance of the synaptic input has little influence on the average firing rate and our model can be greatly simplified. In that case, the F(I)-curve can directly be used as sigmoid, without keeping track of the variances, reducing our approach to that of Shriki et al. [20] who assume a single population with all-to-all connections.

Hutt [24] derived a sigmoidal function in a similar manner as Wilson and Cowan [1], but included the firing rate curve, rather than assuming all-or-none firing neurons. However, he considered the variances of the state of the neurons to be given, while we derived these from the statistics of the recurrent synaptic input.

The approach of Faugeras et al. [25] investigates how network connectivity determines the network dynamics. It considers the same system as we do, i.e. synaptically connected cells that fire instantaneously depending on their input, but is not concerned with the derivation of a specific sigmoid function. Our derivation of the sigmoid could be combined with their more elaborate approach to the network spike rate statistics. This would allow for a rigorous investigation of the parameter regimes in which the dynamics of the network model converge to those of the neural mass equations, i.e. where the mean and variance alone provide an adequate description of the firing rate dynamics. This is not always the case, for example in networks that exhibit avalanches or synchronized firing. More recent work from this group $[26,27]$ is concerned with networks of Hodgkin-Huxley neurons. However, due to the complexity of their derived mean-field equations, that work is more suitable for investigating and proving basic properties of neural networks than for investigating pathologies. 
Deco et al. [12] discuss a more profound method for deriving a NMM, starting from ensemble density models. This method, however, assumes the population dynamics can be described with a diffusion approximation. This is not the case in our networks, because the cells receive heterogeneous input and hence the state of the tonically firing cells is strongly correlated with their input. To model low firing rates, we recommend to adapt the approach from Deco et al. by taking this heterogeneity into account. This allows modeling of pathological populations with relatively low firing rates, for which the NMM we presented is not valid.

\subsection{Conclusion}

We have presented a new neural mass model that is fully derived from physiological expressions. It was shown how a sigmoidal function can be calculated from the F(I)-curves of the single cells and the variances of the firing rates. Additionally, we presented expressions for the dynamics of the variances of the neuronal firing rates and synaptic conductances.

We verified this model by showing that it excellently reproduces the dynamics observed in a network of heterogeneously coupled excitatory and inhibitory $\mathrm{HH}-$ neurons. The time courses of the means and standard deviations of both the firing rates and the synaptic conductances are all described accurately. Furthermore, it was discussed that the model is valid as long as the synaptic conductances fluctuate on a time scale similar to or slower than the neurons' spike rates, and the modal firing rates are higher than the frequencies of the dynamics of interest.

Besides yielding the variance of the firing rates and input currents in the populations, our approach allows for investigating how alterations of the single cell dynamics affect the macroscopic activity. The firing rate curve of the single cell serves as link between the two. For pathologically and pharmacologically induced changes this curve can be calculated directly with a biophysical single cell model, without the need for reducing the model to a simpler form first. We demonstrated this for an increased extracellular potassium concentrations. A wide range of alterations of the single cell dynamics, for example by channel blockers, can be modeled in the same way.

\section{A Dynamics and distribution of the synaptic input in a network of spiking cells}

A network with excitatory and inhibitory neurons, contains four sets of synaptic connections ( $\mathrm{e} \rightarrow \mathrm{e}, \mathrm{e} \rightarrow \mathrm{i}, \mathrm{i} \rightarrow \mathrm{e}$ and $\mathrm{i} \rightarrow \mathrm{i}$ ). In this appendix, we describe the total synaptic conductance $G_{n}$ induced by one of these sets on a postsynaptic neuron $n$ (see fig- 


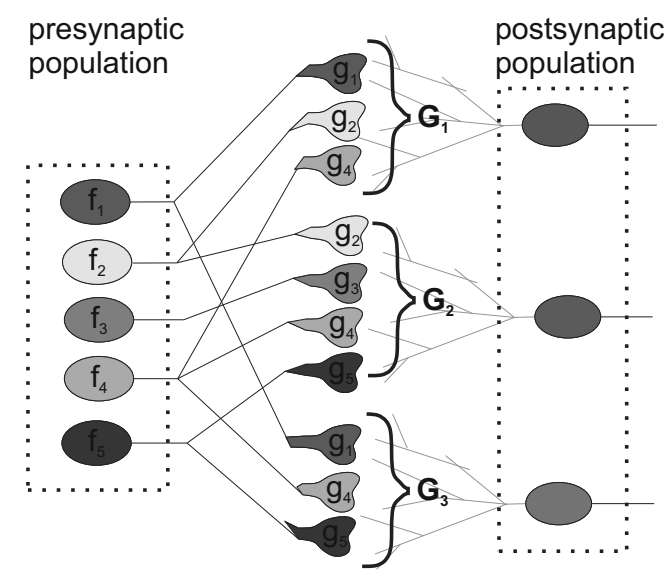

Figure 6.16: Sketch of the synaptic variables. One synaptic population is shown, with presynaptic single cell firing rates $f_{m}$, synaptic conductances $g_{m}$ and summed synaptic conductances $G_{n}$.

ure 6.16). First, the distribution of $G$ is derived from a synaptic connection matrix. We denote the number of cells in the presynaptic population with $M$, and that in the postsynaptic one with $N$. This yields a maximum of $N \times M$ synapses in the set. The presence and strength of the synaptic connections is described with an $N \times M$ matrix $W . W_{n, m}=1$ denotes a synaptic connection from cell $m$ to $n$ of average weight, while 0 stands for no connection. This system is equal to that analyzed by Faugeras et al. [25], however we do not constrain our analysis to a normal distribution of $W_{n, m}$.

We assume the states of the synapses originating from the same presynaptic cell $m$ are all equal, except for their weight. They are therefore described with the same variable $g_{m}(t)$. The conductance of the synapse $g_{m}(t)$ is described as the convolution of the impulse response $H$ with the spike rate $f_{m}(t)$ of cell $m$ :

$$
g_{m}(t)=H * f_{m}(t) .
$$

The total synaptic conductance $G_{n}$ of cell $n$ induced by one presynaptic population, is calculated as the linear sum of all synapses, using the connection matrix:

$$
G_{n}(t)=\sum_{m} W_{n, m} g_{m}(t)
$$

Assuming the firing rates and synaptic strengths are uncorrelated, and defining the weighted number of connections neuron $n$ receives as

$$
N_{\mathrm{syn}, \mathrm{n}}:=\sum_{m} W_{n, m}
$$


the mean of the total synaptic conductance $\bar{G}$ is readily calculated:

$$
\bar{G}(t)=\bar{N}_{\text {syn }} \bar{g}(t)=\bar{N}_{\text {syn }} H * \bar{f}(t),
$$

, using bars to denote the mean values.

This derivation may seem an unnecessarily elaborate way of obtaining the familiar Eq. 6.28, but it sets the stage for deriving the variance of $G$.

To calculate the variance of $G_{n}$ (Eq. 6.26) over the N postsynaptic cells, the terms $W_{n, m}$ and $g_{m}$ are written as their mean plus a deviation:

$$
\begin{aligned}
\bar{W}_{n} & :=\frac{1}{M} \sum_{m} W_{n, m} \\
\Delta W_{n, m} & :=W_{n, m}-\bar{W}_{n}
\end{aligned}
$$

and similar for g. With these expressions, the variance of $G_{n}$ (Eq. 6.26) is calculated:

$$
\begin{aligned}
\sigma_{G}^{2}: & =\operatorname{var}_{n}\left(G_{n}\right)=\operatorname{var}_{n}\left(\sum_{m} W_{n, m} g_{m}\right) \\
& =\operatorname{var}_{n}\left(\sum_{m}\left(\bar{W}_{n}+\Delta W_{n, m}\right)\left(\bar{g}+\Delta g_{m}\right)\right) \\
& =\operatorname{var}_{n}\left(\bar{g} \sum_{m} \Delta W_{n, m}+\bar{W} \sum_{m} \Delta g_{m}+M \bar{W}_{n} \bar{g}+\sum_{m} \Delta W_{n, m} \Delta g_{m}\right) .
\end{aligned}
$$

The subscript $n$ denotes the variance is calculated over $n$. The first two terms are zero, since the sums over the deltas are per definition zero. Hence,

$$
\sigma_{G}^{2}=\operatorname{var}_{n}\left(M \bar{W}_{n} \bar{g}+\sum_{m} \Delta W_{n, m} \Delta g_{m}\right)
$$

Assuming the synaptic weights and synaptic activations are uncorrelated and using the standard rules for the variance of products and sums:

$$
\sigma_{G}^{2}=\operatorname{var}\left(M \bar{W}_{n}\right) \bar{g}^{2}+\left(\sum_{m} \operatorname{var}_{n}\left(W_{n, m}\right)\right) \operatorname{var}_{m}\left(g_{m}\right)
$$

, noting that $M \bar{W}=N_{\text {syn }}$ (equation 6.27), $\operatorname{var}_{m}\left(g_{m}\right) \equiv \sigma_{g}^{2}$ and defining

$$
N_{\mathrm{syn}}^{\prime}:=\sum_{m} \operatorname{var}_{n}\left(\Delta W_{n, m}\right),
$$

this is results in:

$$
\sigma_{G}^{2}=\operatorname{var}\left(N_{\mathrm{syn}}\right) \bar{g}^{2}+N_{\mathrm{syn}}^{\prime} \sigma_{g}^{2}
$$


The first term is the variance due to differences in the weighted number of synaptic connections $N_{\text {syn }}$ received by the cells. The second term is the variance due to different synaptic activations caused by differences in presynaptic spike rates. $N_{\text {syn }}^{\prime}$ is, for example, equal to $M\left(p-p^{2}\right)$ in the case where cells are randomly connected with probability $p$ and can be interpreted as the average number of connections a postsynaptic neuron does not share with a random other cell of its population.

Now the task to describe the mean and variance of $g(t)$ is left. To calculate $\sigma_{g}$ over the individual synapses, we need to make some assumptions on the distribution of the spikes in the input. Amit and Brunel calculated this variance in the steady state, assuming Poisson (shot noise) statistics [13]. We will show a similar approach can be used for dynamic input and describe statistics for periodically generated action potentials, i.e. with regular intervals. These describe the firing rates observed in our spiking network model better.

There is a fundamental difference between calculating the distribution of the conductance induced by a Poisson process or by a periodical process. The conductance of a synapse receiving action potentials generated by a Poisson process with a known rate, is a stochastic signal. In contrast, the conductance of a synapse receiving action potentials periodically at a known rate is deterministic (assuming the synaptic integration smooths the input sufficiently, such that the phase of the input is irrelevant). Therefore, the variance of the synaptic conductances is induced by the variance of the firing rates themselves, rather than by the realization of the spike generation process. We calculate this variance now for time dependent firing rates.

The firing rates $f(t)$ are considered to be distributed over the presynaptic neurons, with mean $\bar{f}(t)$ and standard deviation $\sigma_{f}(t)$. We consider the inputs to be smooth continuous functions (rates) rather than delta pulse trains, as is common in neural mass modeling.

The mean of the synaptic activation is trivial to calculate from Equation 6.25:

$$
\bar{g} \equiv<g>=<H * f>=H * \bar{f}
$$

, where the brackets denote the expectancy.

To calculate the variance of $g$, we use hats to denote the deviation from the expectancy of $f$ and $g$, i.e.

$$
\hat{g}:=g-\bar{g},
$$

and explicitly write the convolutions as integrals:

$$
\begin{aligned}
\sigma_{g}^{2} & \equiv<\hat{g}^{2}>=<(H * \hat{f}(t))^{2}> \\
& =<\left(\int_{0}^{\infty} \hat{f}(t-u) H(u) \mathrm{d} u\right)^{2}> \\
& =\int_{0}^{\infty} \int_{0}^{\infty}<\hat{f}(t-u) \hat{f}\left(t-u^{\prime}\right)>H(u) H\left(u^{\prime}\right) \mathrm{d} u \mathrm{~d} u^{\prime}
\end{aligned}
$$


The expectancy is replaced by the (auto)correlation coefficient $C_{\mathrm{ac}}$ :

$$
\begin{aligned}
C_{\mathrm{ac}}\left(x_{1}, x_{2}\right) & :=\frac{<x_{1} x_{2}>}{\sigma_{x 1} \sigma_{x 2}} \\
\sigma_{g}^{2} & =\int_{0}^{\infty} \int_{0}^{\infty} C_{\mathrm{ac}}\left(\hat{f}(t-u), \hat{f}\left(t-u^{\prime}\right)\right) \sigma_{f}(t-u) \sigma_{f}\left(t-u^{\prime}\right) H(u) H\left(u^{\prime}\right) \mathrm{d} u \mathrm{~d} u^{\prime}
\end{aligned}
$$

In our simulations of spiking networks it was observed that $\hat{f}$ is highly correlated with itself over periods much longer than the duration of $H$, i.e. cells tend to fire faster or slower than the population average over longer periods of time. In that case $C_{\mathrm{ac}} \approx 1$ and the right hand side of equation 6.46 is equal to the squared convolution with the variance (c.f. equations 6.42-6.44),

$$
\sigma_{g}(t) \approx H * \sigma_{f}(t)
$$

which is used for our simulations.

For cases where $\hat{f}$ does fluctuate faster (typically when $\operatorname{var}\left(N_{\text {syn }}\right)$ is low), equation 6.46 can be simplified in another way. It is reasonable to assume the autocorrelation coefficient depends only on the time difference $u-u^{\prime}$, while furthermore $\sigma_{f}$ fluctuates slowly compared to the synaptic time constant. In that case, the autocorrelation coefficient can be effectively replaced with a correction constant $C$ between 0 and 1 . The expression for $\sigma_{g}$ becomes:

$$
\sigma_{g}(t)=C H * \sigma_{f}(t),
$$

where

$$
C=\frac{\int_{0}^{\infty} \int_{0}^{\infty} \exp \left(C_{\mathrm{ac}}\left(u-u^{\prime}\right)\right) \sigma_{f}(t-u) \sigma_{f}\left(t-u^{\prime}\right) H(u) H\left(u^{\prime}\right) \mathrm{d} u \mathrm{~d} u^{\prime}}{\left(\int_{0}^{\infty} H(u) d u\right)^{2}} .
$$

However, deriving a closed expression for the autocorrelation coefficient $C_{\mathrm{ac}}\left(u-u^{\prime}\right)$ in a recurring network is complicated and outside the scope of this work.

\section{References}

[1] H. R. Wilson and J. D. Cowan, "Excitatory and inhibitory interactions in localized populations of model neurons.", Biophysical journal 12, 1-24 (1972).

[2] R. Hindriks and M. J. a. M. van Putten, "Meanfield modeling of propofol-induced changes in spontaneous EEG rhythms.”, NeuroImage 60, 2323-34 (2012).

[3] J. D. Victor, J. D. Drover, M. M. Conte, and N. D. Schiff, "Mean-field modeling of thalamocortical dynamics and a model-driven approach to eeg analysis.", Proc Natl Acad Sci U S A 108 Suppl 3, 15631-15638 (2011). 
[4] A. Hutt, "The anesthetic propofol shifts the frequency of maximum spectral power in eeg during general anesthesia: analytical insights from a linear model.”, Front Comput Neurosci 7, 2 (2013).

[5] M. C. Tjepkema-Cloostermans, R. Hindriks, J. Hofmeijer, and M. J. A. M. van Putten, "Generalized periodic discharges after acute cerebral ischemia: Reflection of selective synaptic failure?", Clin Neurophysiol (2013).

[6] J. P. Dreier, "The role of spreading depression, spreading depolarization and spreading ischemia in neurological disease.", Nature medicine 17, 439-47 (2011).

[7] M. Bazhenov, I. Timofeev, F. Frhlich, and T. J. Sejnowski, "Cellular and network mechanisms of electrographic seizures.”, Drug Discov Today Dis Models 5, 45-57 (2008).

[8] M. H. Meisler and J. A. Kearney, "Sodium channel mutations in epilepsy and other neurological disorders.”, J Clin Invest 115, 2010-2017 (2005).

[9] G. G. Somjen, "Mechanisms of spreading depression and hypoxic spreading depression-like depolarization.”, Physiol Rev 81, 1065-1096 (2001).

[10] D. T. J. Liley, P. J. Cadusch, and M. P. Dafilis, "A spatially continuous mean field theory of electrocortical activity.”, Network (Bristol, England) 13, 67-113 (2002).

[11] G. Holt, "A critical reexamination of some assumptions and implications of cable theory in neurobiology", Ph.D. thesis, California Institute of Technology (1997), URL http://lnc.usc.edu/ holt/papers/thesis/.

[12] G. Deco, V. K. Jirsa, P. a. Robinson, M. Breakspear, and K. Friston, "The dynamic brain: from spiking neurons to neural masses and cortical fields.”, PLoS computational biology 4, e1000092 (2008).

[13] D. Amit and N. Brunel, "Dynamics of a recurrent network of spiking neurons before and following learning", Network: Computation in Neural Systems (1997).

[14] B.-J. Zandt, B. ten Haken, J. G. van Dijk, and M. J. A. M. van Putten, "Neural dynamics during anoxia and the "wave of death"”, PLoS One 6, e22127 (2011).

[15] J. Ziburkus, J. R. Cressman, E. Barreto, and S. J. Schiff, "Interneuron and pyramidal cell interplay during in vitro seizure-like events.”, J Neurophysiol 95, 3948-3954 (2006).

[16] B. H. Jansen and V. G. Rit, "Electroencephalogram and visual evoked potential generation in a mathematical model of coupled cortical columns.”, Biol Cybern 73, 357-366 (1995).

[17] P. A. Robinson, C. J. Rennie, J. J. Wright, H. Bahramali, E. Gordon, and D. L. Rowe, "Prediction of electroencephalographic spectra from neurophysiology.”, Phys Rev E Stat Nonlin Soft Matter Phys 63, 021903 (2001).

[18] A. Chizhov and L. Graham, "Population model of hippocampal pyramidal neurons, linking a refractory density approach to conductance-based neurons", Physical Review E 75, 011924 (2007).

[19] S. Ostojic and N. Brunel, "From spiking neuron models to linear-nonlinear models.", PLoS computational biology 7, e1001056 (2011).

[20] O. Shriki, D. Hansel, and H. Sompolinsky, "Rate models for conductance-based cortical neuronal networks.", Neural computation 15, 1809-41 (2003). 
[21] C. Allen and C. F. Stevens, "An evaluation of causes for unreliability of synaptic transmission.”, Proc Natl Acad Sci U S A 91, 10380-10383 (1994).

[22] P. a. Robinson, H. Wu, and J. W. Kim, "Neural rate equations for bursting dynamics derived from conductance-based equations.", Journal of theoretical biology 250, 663-72 (2008).

[23] S. Visser, "From spiking neurons to brain waves", Ph.D. thesis, University of Twente, Enschede (2013), URL http://doc . utwente.nl/85269/.

[24] A. Hutt, "The population firing rate in the presence of GABAergic tonic inhibition in single neurons and application to general anaesthesia.", Cognitive neurodynamics 6, 227-37 (2012).

[25] O. Faugeras, J. Touboul, and B. Cessac, "A constructive mean-field analysis of multi-population neural networks with random synaptic weights and stochastic inputs.”, Frontiers in computational neuroscience 3, 1 (2009).

[26] J. Touboul, G. Hermann, and O. Faugeras, "Noise-induced behaviors in neural mean field dynamics", SIAM Journal on Applied Dynamical Systems 11, 49-81 (2012).

[27] J. Baladron, D. Fasoli, O. Faugeras, and J. Touboul, "Mean-field description and propagation of chaos in networks of hodgkin-huxley and fitzhugh-nagumo neurons.", J Math Neurosci 2, 10 (2012). 


\section{7 \\ General Discussion and Outlook}

Ischemic and hypoxic damage results from a complex process, involving many different physiological dynamics which evolve on multiple time and length scales. Metabolism, blood flow, the neuronal membrane voltage, glial activity, synapses and the constitution of the intra- and extracellular space all interact. The two processes that determine energy consumption and excitotoxicity, the dynamics of ion concentrations and the neuronal membrane voltage, were investigated in detail in this thesis.

\subsection{Ischemia in the rat and human brain}

The work in this thesis was performed with investigating ischemia/hypoxia in the human brain in mind. Experimental data and parameter values, however, are mostly obtained from rat. Rat neural tissue is similar to human neural tissue, and in both the same processes take place, e.g. neuronal firing, ion homeostasis by the $\mathrm{Na} / \mathrm{K}$ pump, ATP generation in the mitochondria. However, physiological parameters are different, e.g. resting ion concentrations, neuron density, white/grey matter ratio. Experimental data can validate a model with parameters for rat, and subsequently the parameters can be changed to investigate the dynamics in human tissue. Since the dynamics of ischemic damage are complex, changing the parameters can result in different dynamical behavior, i.e. a different progression of ischemic damage, in which different processes have a key role.

Ischemia in rat and human tissue can be modeled with the same equations, using different parameters. Therefore, the work described in this thesis is applicable to both 
rat and human patients.

\subsection{Wave of death}

The dynamic behavior of ion concentrations in and outside the neurons is modeled and describe during failure of ion homeostasis due to hypoxia/ischemia. During energy deprivation (anoxia, ischemia) the net transmembrane potassium and sodium fluxes lead to membrane depolarization (chapter 3 and 5) and spreading depression (chapter 4). This process of depolarization has been described and mathematically modeled before [1-4]. A new contribution of this thesis was to identify anoxic depolarization as the physiological substrate of the "wave of death". The sudden depolarization is observed as a large amplitude wave in the EEG when recording with a standard band-pass filter. Therefore, the wave does not reflect neuronal cell death, but massive depolarization, which is in principle reversible. The phenomenon is interesting for two reasons.

First, prolonged depolarization causes cell swelling, as well as a large $\mathrm{Ca}^{2}+$ influx, eventually leading to apoptosis. Therefore, the depolarization wave does signal the transition to a highly unfavorable state of the ion gradients, that will eventually result in cell swelling and neuronal death if not restored sufficiently fast. Second, the state following cerebral depolarization can be viewed as the ultimate form of unconsciousness: not only information processing and transfer between brain areas is absent, as for example during anesthesia, but all neuronal activity has ceased.

Recently, similar experiments on rats have been performed with multiple EEG electrodes [5]. Cardiac arrest was induced by intracardial $\mathrm{KCl}$ injection, and the power spectrum of the resulting EEG signals was analyzed. In these measurements, temporary activity was observed in the low gamma band $(25-55 \mathrm{~Hz})$ after a period of electrical silence. Another new finding was the large coherence between the signals at different electrodes. If this coherence is not an artifact and indeed stems from localized sources in the brain, some communication mechanisms must still be intact. Candidate mechanisms are electrical field and ephaptic transmission, gapjunction coupling and remaining synaptic function. The relation with consciousness and near-death experience of this temporary burst of activity and coherence before and during depolarization is still being investigated. Loss of coherence is associated with unconsciousness. However, its presence does not necessarily imply consciousness. Given the highly pathological state of the brain in this state, normal information processing is unlikely. 


\subsection{Spreading depolarization}

A second result is the reduction of the dynamics of a single neuron and the ion concentrations in the extracellular space, to model hypoxic spreading depolarization. The reduced model expulses potassium at constant rate when above a concentration threshold, while removing it at a linear rate. This threshold was determined to be the concentration above which the cells generate action potentials without receiving synaptic input. The expulsion and removal rate were used in a reaction-diffusion equation that models the triggering and propagation of spreading depolarization. Similar macroscopic descriptions have been used before to model SD propagation [6-8]. However, with this reduction an explicit connection has been made between the single neuron parameters (e.g. channel conductances, pump rates) and the macroscopic parameters of SD (e.g. expulsion rate, concentration threshold).

In the hours to days following ischemia, spreading depolarizations (SD) or periinfarct depolarizations (PID) occur around the infarct [9-12]. Measurements in patients indicate SD's/PID's indeed increase infarct size and exacerbate neural damage $[13,14]$. Therefore SD's are a potential target for therapy, for example by channel blockers or hypothermia. With the combination of the reaction-diffusion model and single cell model, the effects of channel blockers on the propagation of SD in the penumbra can be investigated. When potassium is removed rapidly compared to the expulsion rate, SD cannot propagate, for example (equation 4.37). With the constructed reaction-diffusion model, the occurrence and propagation of the first SD can be predicted. Further work is necessary to relate phenomenological expressions for the dynamics of recovery and refractiveness and the resulting generation of multiple SD's, e.g. [8, 15], to models of the single cell dynamics $[3,16]$.

Experimental data was used to validate the derived expressions. The dynamics of the single cell model, that was used to derive the release rate of potassium, were qualitatively validated with experimental measurements in vitro (chapter 5). The wave shape predicted by the model of SD propagation (chapter 3) was validated from the time courses of potassium during SD from literature. Furthermore, new experiments were performed measuring the electrical activity, blood flow and extracellular potassium concentration in in vivo cortex of rat. The aim was to determine the threshold concentration for neuronal depolarization, and determine the relation with the bending point of the time course of the potassium concentration during SD. This could determine the relative contribution of other substances and mechanisms besides diffusion of potassium to the propagation. Although very instructive for me personally, the results of the experiments were inconclusive [17]. 


\subsection{Experimental validation of the Hodgkin-Huxley model during depolarization}

Various models of single neurons with dynamic ion concentrations have been previously published, including various ion channels and neuron morphologies $[2-4,18-$ 20]. The membrane voltage dynamics of the $\mathrm{HH}$ model with dynamic ion concentrations used in chapters 3 and 4 was qualitatively validated from experimental measurements in vitro (performed by Tyler Stigen, the Netoff lab, Minnesota, see chapter 5). Analysis of the experimental data shows that, qualitatively, the HH model suffices to describe the membrane dynamics and bifurcation structure of mammalian pyramidal cells during depolarization. This is not self-evident, since the HH model was developed to describe neuron dynamics under constant, physiological circumstances.

The dynamics of the corresponding ion concentrations, and how these are driven by the ionic transmembrane currents, still need to be validated experimentally. These could not be validated yet, since data on the ion concentrations was not obtained. Ideally, such a validation is done in vivo, since diffusive coupling of the extracellular space with the blood flow is important in homeostasis of the extracellular space. However, a good first step would be to validate a mathematical model without blood flow by further experiments in slices. Measurements of the membrane voltage of a single cell as described in chapter 5 can be combined with measurements of the extracellular potassium and sodium concentrations using ion selective electrodes. To validate that the single cell dynamics correspond to the population activity, these electrodes can be used to measure the extracellular potential as well. Furthermore, to assess how synaptic activity slows or hastens the depolarization process, these same measurements can be performed in a low calcium medium, that inhibits synaptic activity.

\subsection{Neural mass modeling}

A neural mass model (NMM) was proposed (chapter 6), that shows how synaptically coupled populations of inhibitory and excitatory neurons behave for alterations in single cell parameters. The F(I)-curve summarizes the electrophysiology of the single cell and is used to calculate the macroscopic neural activity. Neural mass models have been studied extensively and have been applied to various pathologies in literature [21-24]. However, this is the first work in which the influences of ion concentrations on the cells firing rates are taken into account explicitly.

A neural mass model with variable ion concentrations (chapter 6) can describe the propagation of SD in migraine aura. During a migraine aura, the synapses do not fail before SD occurs, in contrast with hypoxic SD. Therefore, the network dynamics of the cells determine the spike rate and potassium release, rather than the 
single cell dynamics. To investigate the propagation and initiation of SD in migraine, a NMM should be constructed and reduced in a similar way as the HH single cell model (chapter 4). This results in a reaction-diffusion model as presented in chapter 4 , but with a different function for the potassium reaction rate. As for the case without synaptic transmission, SD is prevented by limiting the build-up of extracellular potassium and glutamate, which can be accomplished by stimulating uptake, glial buffering and diffusion or reducing release. Synaptic function allows for an additional approach to prevent SD, by reducing potassium efflux through the population dynamics. Possibly, medication can make the inhibitory cells more sensitive to increases in potassium/glutamate. This limits the firing rates of the pyramidal cells when extracellular potassium/glutamate levels rise, thereby increasing the critical concentration for initiating SD. The repolarization process in migraine aura can be investigated with a single cell model such as [16], since the synapses have been shown to fail after the depolarization [25]. This modeling of normoxic SD can be used to predict the effects of proposed therapies that aim to prevent, stop, or enhance recovery from, a migraine aura.

Furthermore, a NMM with variable ion concentrations can be useful for interpreting the EEG of postanoxic patients. For example, a transition from generalized periodic discharges (GDP's) to a more physiological EEG could reflect the recovery of the inhibitory synapses [22]. Could it alternatively indicate an increased excitability of the inhibitory cells by excitotoxic substances? Analysis of the dynamics of a NMM has the perspective to yield a patient specific estimation of the evolution of the state of the brain. I propose to calculate a bifurcation diagram depending on two or three key parameters, showing the various EEG dynamics and transitions, similar to that of the single cell dynamics presented in chapter 5. To determine the key parameters for patient survival and recovery of neurological function, the processes occurring in the brain after global ischemia and reperfusion that affect the electrophysiology of the neurons must be investigated: (selective) synaptic dysfunction and neuronal death, and the constitution of the extracellular space. Experiments on in vitro and in vivo stroke models could be performed using microdialysis probes, as well as post mortem measurements of (selective) structural damage of the synapses and neurons in patients. Subsequently, the firing rate curves of the pyramidal cells and interneurons must be characterized as a function of the evolving parameters. These curves can be inferred from a computational single cell model, and be validated by in vitro patch clamp data. With these parameter values, the dynamical behavior of the EEG can be calculated from the NMM described in chapter 6 . 


\subsection{Modeling an infarct on multiple scales}

Mathematical analysis of computational models of stroke, may greatly help in designing and predicting the effect of new therapies and medication that potentially prevent secondary cell death. Analysis can elucidate the relation between physiological parameters and e.g. time windows of opportunity and doses necessary for effectiveness. The work in this thesis specifically allows to investigate therapies that prevent excitotoxicity by targeting voltage gated ion channels and synaptic mechanisms. This work allows their effect on the single cell to be expressed in the parameters of a model that describes the tissue macroscopically.

As future work, I propose to construct a two-dimensional model of infarcted cortical tissue in the sub-acute phase (first hours to days). This model should describe local ion homeostasis, neural activity, and energy availability, as well as the interaction between parts of the tissue by diffusion of ions and neurotransmitters. Similar macroscopic models are already available [15, 26], however these use dimensionless, phenomenological expressions and are not explicitly connected to physiological single cell parameters. The parameters of these phenomenological models can be described by simplifying more detailed physiological models.

In the model proposed now, expressions for the rise in extracellular potassium, neural activity and resulting neuronal depolarization can be applied as derived in this thesis (chapters 3, 4 and 6). Reduced expressions for the repolarization process still need to be obtained from single cell parameters. The work of Hübel et al. [16] describing the parameter regimes for a HH-model that allow for repolarization can be used as starting point for this. Chapter 2 presented basic calculations of the energy consumption and supply around an infarct as a function of neural activity and blood flow. The model of Cloutier et al. [27] can be used to model ATP production.*

The proposed model will produce energy depletion, depolarization of cells and resulting SD's/PID's when a focal restriction of blood flow is imposed. The main goal of such a model is to describe secondary cell death in the penumbra, and how this can be prevented. Therefore, processes that induce damage and cell death must be included subsequently. The proposed model, using in part the work presented in this thesis, lends itself for modeling three important causes of cell death and damage: cell swelling, noxious metabolic products and calcium influx.

- The swelling of a neuron is induced by water movement from the extracellular space into the cell by osmosis, as for example modeled in [28, 29]. Cell volume

${ }^{*}$ As I found out during the course of my $\mathrm{PhD}$ research, in a biological system such as the hypoxic brain, the bottom-up modeling approach often used in physics is problematic for several reasons: the multiple spatial and temporal scales involved, the resulting lack of obvious elementary entities, the large amount of interactions between entities and processes, unavailability and variability of parameter values, and emergent behavior that exhibits many bifurcations within the physiological parameter domain. 
is a function of the intra- and extracellular ion concentrations and is therefore a natural extension of this work.

- Metabolic challenged tissue shows altered metabolism, for example an increased rate of anaerobic glycolysis. Furthermore, tissue temporarily increases its energy consumption following PID's/SD's in order to restore the ion gradients. In both cases, potentially harmful side products are generated, notably reactive oxygen species [30] and $\mathrm{H}^{+}$[31]. The dynamics of the $\mathrm{pH}$ as a function of $\mathrm{Na} / \mathrm{K}$-pump rate and oxygen/glucose supply have been described for cortical tissue [32], while existing models of reactive oxygen species production in ischemic cardiac tissue [33-36] may be modified for the brain.

- Increased intracellular calcium levels result from neuronal depolarization and/or failure of calcium transporters. Intracellular calcium induces mitochondrial damage and apoptosis [37]. Calcium dynamics can be included in the proposed model. An extensive discussion of modeling calcium influx, removal and intracellular buffering is given in [38].

In conclusion, I propose to adapt an existing phenomenological model of an ischemic infarct by including analyzable expressions derived from detailed physiological models. Part of this work has been performed in this thesis, while other expressions can be derived from existing detailed models. With this model, the effects of medication and therapies can be investigated.

\subsection{Closing remarks}

This thesis has described the dynamics of ion concentrations and neuronal activity after ischemia/hypoxia. Combined with knowledge of the dynamics of metabolism and cell damage, from modeling and experiments, this may allow us to interpret the changes in dynamics of the EEG of postanoxic patients, and predict the effects of new neuroprotective therapies and drugs. Plenty of opportunities are still left to increase our fundamental knowledge about ischemic damage, as well as to apply this knowledge to improve patient care.

\section{References}

[1] G. G. Somjen, "Mechanisms of spreading depression and hypoxic spreading depression-like depolarization.”, Physiol Rev 81, 1065-1096 (2001).

[2] H. Kager, W. J. Wadman, and G. G. Somjen, "Simulated seizures and spreading depression in a neuron model incorporating interstitial space and ion concentrations.", J Neurophysiol 84, 495$512(2000)$. 
[3] J. R. Cressman, G. Ullah, J. Ziburkus, S. J. Schiff, and E. Barreto, "The influence of sodium and potassium dynamics on excitability, seizures, and the stability of persistent states: I. single neuron dynamics.", J Comput Neurosci 26, 159-170 (2009).

[4] W. Yao, H. Huang, and R. M. Miura, "A continuum neuronal model for the instigation and propagation of cortical spreading depression.”, Bull Math Biol 73, 2773-2790 (2011).

[5] J. Borjigin, U. Lee, T. Liu, D. Pal, S. Huff, D. Klarr, J. Sloboda, J. Hernandez, M. M. Wang, and G. A. Mashour, "Surge of neurophysiological coherence and connectivity in the dying brain.", Proc Natl Acad Sci U S A 110, 14432-14437 (2013).

[6] B. Grafstein, Neuronal release of potassium during spreading depression., chapter Brain Function, Vol. I. (University of California Press, Berkeley, Calif. 87.) (1963).

[7] M. Dahlem and S. Muller, "Reaction-diffusion waves in neuronal tissue and the window of cortical excitability", Annalen der Physik 13, 442-449 (2004).

[8] M. A. Dahlem, R. Graf, A. J. Strong, J. P. Dreier, Y. A. Dahlem, M. Sieber, W. Hanke, K. Podoll, and E. Schll, "Two-dimensional wave patterns of spreading depolarization: Retracting, re-entrant, and stationary waves", Physica D: Nonlinear Phenomena 239, 889 - 903 (2010).

[9] C. Drenckhahn, M. K. L. Winkler, S. Major, M. Scheel, E.-J. Kang, A. Pinczolits, C. Grozea, J. A. Hartings, J. Woitzik, J. P. Dreier, and C. O. S. B. I. D. s. g. , "Correlates of spreading depolarization in human scalp electroencephalography.", Brain 135, 853-868 (2012).

[10] J. P. Dreier, "The role of spreading depression, spreading depolarization and spreading ischemia in neurological disease.”, Nat Med 17, 439-447 (2011).

[11] M. Lauritzen, J. P. Dreier, M. Fabricius, J. A. Hartings, R. Graf, and A. J. Strong, "Clinical relevance of cortical spreading depression in neurological disorders: migraine, malignant stroke, subarachnoid and intracranial hemorrhage, and traumatic brain injury.", J Cereb Blood Flow Metab 31, 17-35 (2011).

[12] C. Dohmen, O. W. Sakowitz, M. Fabricius, B. Bosche, T. Reithmeier, R.-I. Ernestus, G. Brinker, J. P. Dreier, J. Woitzik, A. J. Strong, R. Graf, and C.-O. S. o. B. I. D. C. O. S. B. I. D. , "Spreading depolarizations occur in human ischemic stroke with high incidence.", Ann Neurol 63, 720-728 (2008).

[13] H. Nakamura, A. J. Strong, C. Dohmen, O. W. Sakowitz, S. Vollmar, M. Su, L. Kracht, P. Hashemi, R. Bhatia, T. Yoshimine, J. P. Dreier, A. K. Dunn, and R. Graf, "Spreading depolarizations cycle around and enlarge focal ischaemic brain lesions.”, Brain 133, 1994-2006 (2010).

[14] J. P. Dreier, J. Woitzik, M. Fabricius, R. Bhatia, S. Major, C. Drenckhahn, T.-N. Lehmann, A. Sarrafzadeh, L. Willumsen, J. A. Hartings, O. W. Sakowitz, J. H. Seemann, A. Thieme, M. Lauritzen, and A. J. Strong, "Delayed ischaemic neurological deficits after subarachnoid haemorrhage are associated with clusters of spreading depolarizations.”, Brain 129, 3224-3237 (2006).

[15] K. Revett, E. Ruppin, S. Goodall, and J. A. Reggia, "Spreading depression in focal ischemia: a computational study.”, J Cereb Blood Flow Metab 18, 998-1007 (1998).

[16] N. Hübel, E. Schöll, and M. A. Dahlem, "Bistable dynamics of ion homeostasis in ion-based neuron models", ArXiv e-prints (2013). 
[17] B. Zandt, D. Feuerstein, H. Backes, H. Ima, B. ten Haken, M. van Putten, and R. Graf, "The role of diffusing potassium in spreading depolarization (poster)", BRAIN2013, XXVIth International Symposium on Cerebral Blood Flow, Metabolism and Function, May 20-23 2013, Shanghai.

[18] G. P. Krishnan and M. Bazhenov, "Ionic dynamics mediate spontaneous termination of seizures and postictal depression state.", J Neurosci 31, 8870-8882 (2011).

[19] L. yehaug, I. stby, C. M. Lloyd, S. W. Omholt, and G. T. Einevoll, "Dependence of spontaneous neuronal firing and depolarisation block on astroglial membrane transport mechanisms.", J Comput Neurosci 32, 147-165 (2012).

[20] S. Ching, P. L. Purdon, S. Vijayan, N. J. Kopell, and E. N. Brown, "A neurophysiologicalmetabolic model for burst suppression.”, Proc Natl Acad Sci U S A 109, 3095-3100 (2012).

[21] G. Deco, V. K. Jirsa, P. A. Robinson, M. Breakspear, and K. Friston, "The dynamic brain: from spiking neurons to neural masses and cortical fields.”, PLoS Comput Biol 4, e1000092 (2008).

[22] M. C. Tjepkema-Cloostermans, R. Hindriks, J. Hofmeijer, and M. J. A. M. van Putten, "Generalized periodic discharges after acute cerebral ischemia: Reflection of selective synaptic failure?", Clin Neurophysiol (2013).

[23] R. Hindriks and M. J. A. M. van Putten, "Meanfield modeling of propofol-induced changes in spontaneous eeg rhythms.”, Neuroimage 60, 2323-2334 (2012).

[24] A. Aarabi and B. He, "Seizure prediction in hippocampal and neocortical epilepsy using a modelbased approach", Clin Neurophysiol (2014).

[25] B. E. Lindquist and C. W. Shuttleworth, "Adenosine receptor activation is responsible for prolonged depression of synaptic transmission after spreading depolarization in brain slices.", Neuroscience 223, 365-376 (2012).

[26] L. Vatov, Z. Kizner, E. Ruppin, S. Meilin, T. Manor, and A. Mayevsky, "Modeling brain energy metabolism and function: a multiparametric monitoring approach.”, Bull Math Biol 68, 275-291 (2006).

[27] M. Cloutier, F. B. Bolger, J. P. Lowry, and P. Wellstead, "An integrative dynamic model of brain energy metabolism using in vivo neurochemical measurements.", J Comput Neurosci 27, 391-414 (2009).

[28] M.-A. Dronne, J.-P. Boissel, and E. Grenier, "A mathematical model of ion movements in grey matter during a stroke.", J Theor Biol 240, 599-615 (2006).

[29] M.-A. Dronne, E. Grenier, G. Chapuisat, M. Hommel, and J.-P. Boissel, "A modelling approach to explore some hypotheses of the failure of neuroprotective trials in ischemic stroke patients.", Progress in biophysics and molecular biology 97, 60-78 (2008).

[30] A. Viggiano, E. Viggiano, I. Valentino, M. Monda, A. Viggiano, and B. De Luca, "Cortical spreading depression affects reactive oxygen species production.”, Brain Res 1368, 11-18 (2011).

[31] W. A. Mutch and A. J. Hansen, "Extracellular ph changes during spreading depression and cerebral ischemia: mechanisms of brain ph regulation.”, J Cereb Blood Flow Metab 4, 17-27 (1984).

[32] P. Orlowski, M. Chappell, C. S. Park, V. Grau, and S. Payne, "Modelling of ph dynamics in brain cells after stroke.", Interface Focus 1, 408-416 (2011). 
[33] S. Cortassa, M. A. Aon, E. Marbn, R. L. Winslow, and B. O'Rourke, "An integrated model of cardiac mitochondrial energy metabolism and calcium dynamics.”, Biophys J 84, 2734-2755 (2003).

[34] S. Cortassa, M. A. Aon, R. L. Winslow, and B. O'Rourke, “A mitochondrial oscillator dependent on reactive oxygen species.”, Biophys J 87, 2060-2073 (2004).

[35] J. M. Kembro, M. A. Aon, R. L. Winslow, B. O'Rourke, and S. Cortassa, "Integrating mitochondrial energetics, redox and ros metabolic networks: a two-compartment model.", Biophys J 104, 332-343 (2013).

[36] A.-C. Wei, M. A. Aon, B. O'Rourke, R. L. Winslow, and S. Cortassa, "Mitochondrial energetics, ph regulation, and ion dynamics: a computational-experimental approach.", Biophys J 100, 2894-2903 (2011).

[37] K.-A. Hossmann, "Pathophysiology and therapy of experimental stroke.", Cell Mol Neurobiol 26, 1057-1083 (2006).

[38] E. de Schutter and P. Smolen, "Calcium dynamics in large neuronal models", in Methods in neuronal modeling, 2nd ed., edited by C. Koch and I. Segev, 211-250 (MIT Press, Cambridge) (1998). 


\section{Summary}

The interruption of blood flow to the brain as occurs in cardiac arrest and stroke results within minutes in irreversible damage. This leads to an estimated 17 million deaths per year worldwide and leaves many disabled. The development of neuroprotective treatments that prevent cell damage after stroke has so far largely been unsuccessful, while we still have an incomplete understanding of the dynamics of the processes involved. This thesis has focused on the dynamics of ion concentrations and neuronal activity during and after hypoxia. Pump rates lower when ATP dependent ion transporters are insufficiently supplied with energy, causing transmembrane ion gradients to diminish, with significant effects on neuronal activity and function. Neuronal activity in turn determines the rate at which ions flow between the intraand extracellular space, thereby creating a dynamically interacting system.

In chapter 3, it was shown that the extracellular potassium concentration has a crucial role in anoxic depolarization. If potassium fluxes are insufficiently compensated by the ATP-dependent $\mathrm{Na} / \mathrm{K}$ pumps, the extracellular concentration may reach a critical concentration, and neurons will autonomously generate action potentials, resulting in an additional, significant increase in potassium efflux. This efflux induces massive depolarization of the neurons, reflected as a transient wave of activity on the scalp EEG. This explains the "Wave of Death" that is observed in rats after decapitation. The EEG initially becomes flat after approximately $10 \mathrm{~s}$, but a large amplitude wave occurs "paradoxically" approximately a minute later. As argued, this is the response of healthy neuronal membranes to changes in ion gradients, and does not reflect cell death. It does signal the transition to a highly unfavorable state, which will lead to cell damage soon after due to swelling and calcium influx.

The released potassium does not only excite the neurons that released it, but also diffuses to neighboring cells, causing a chain reaction or "reaction-diffusion" process. Chapter 4 has presented expressions for idealized spreading depolarization (SD). It was assumed that propagation is purely mediated by diffusion of a substance (potassium or glutamate), which is released at constant rate when its concentration is above a fixed threshold. Obtained expressions describe the initiation, propagation and wave shape in four parameters that have a clear physiological interpretation. The predicted shape of the onset of the wave was validated with potassium measurements in vivo in rat from literature. This framework may help, in combination with experiments, in 
determining the role of diffusible substances in the propagation of SD and how this can be affected by hypoxia and/or pharmaceutical interventions that aim to prevent peri-infarct depolarizations and migraine auras.

The model used to calculate the behavior of depolarizing single neurons in the previous two chapters, the Hodgkin-Huxley ( $\mathrm{HH}$ ) model with dynamic ion concentrations, is experimentally validated in Chapter 5. Several time courses of depolarizing pyramidal cells were obtained in in-vitro experiments after blocking the $\mathrm{Na} / \mathrm{K}$-pump with ouabain. Five different types of membrane voltage dynamics were observed. These correspond to different trajectories of the sodium and potassium Nernst potentials in a bifurcation diagram of the $\mathrm{HH}$ model. These experiments serve as a qualitative validation of the dynamics predicted by the model.

In chapter 6, an initial effort was made in modeling the behavior of large populations of neurons (neural mass models), rather than single ones, with pathological transmembrane ion gradients. The firing rate curve was used as link between the single cell and neural mass model. Additionally, the variance of the input currents over the cells in a population was determined from the network connectivity and spike rates. This model excellently reproduces the dynamics observed in a simulated network of HH-neurons, provided the synaptic conductances fluctuate on a time scale similar to or slower than the neurons' spike rates and the modal firing rates are higher than the frequencies of the dynamics of interest. The firing rate curve can easily be calculated from a HH model for various ion concentrations and ion channel conductances. This allows for the analysis of the macroscopic activity affected by channel blockers and pathological ion concentrations, thus allowing simulations of dynamics in hypoxic conditions and pharmacological interventions. 


\section{Samenvatting}

Deze thesis beschrijft de rol van ionconcentraties en neurale activiteit bij hypoxie en ischemie in het brein. Door energiegebrek faalt de homeostase van ionconcentraties en verandert de neuronale dynamica. De depolarisatie van neuronen die hierdoor tijdelijk op kan treden, blokkeert niet alleen de neurale functie, maar kan uiteindelijk ook leiden tot celdood en massale hersenschade.

Hoofdstuk 3 behandelt een direct meetbaar effect van een complete stop van de ionpompen. In experimenten met ratten (van Rijn et al, PLoS One 6, e16514, 2011) vertoonde het elektro-encefalogram (EEG) een plotselinge golf met hoge amplitude, een minuut na onthoofding. Er werd gespeculeerd dat deze golf duidt op een plotselinge dood van de neuronen in het brein. Een computationeel model van een enkel neuron en zijn intra- en extracellulaire ionconcentraties laat het fysiologisch mechanisme zien achter deze golf: het stoppen van de $\mathrm{Na} / \mathrm{K}$-pomp leidt tot het lekken van kalium uit de cel. Boven een kritieke grens leidt tot de generatie van actie potentialen, die snel kalium vrij laten komen. Dit veroorzaakt een plotselinge depolarisatie. In combinatie met een hoog doorlaat-filter zorgt dit voor de golf in het EEG. Dit proces is niet noodzakelijkerwijs irreversibel.

In hoofdstuk 4 wordt verspreidende depolarisatie (spreading depolarization, SD) behandeld, een fenomeen wat een belangrijke rol speelt bij een beroerte en migraine. Ondanks deze belangrijke rol zijn de onderliggende processen van de propagatie van SD slecht begrepen. Een versimpeld model dat zowel fysiologisch is als kwantitatief wordt beschreven. De concentraties van excitatoire substanties (glutamaat en kalium) tijdens de start van SD kunnen worden beschreven met een reactie-diffusie vergelijking. Oplossingen van deze vergelijking beschrijven de golfvorm, de voortplantingssnelheid en de susceptibiliteit van het weefsel voor SD, in slechts vier parameters: de snelheden waarmee kalium/glutamaat vrijkomen en weer opgenomen worden, de concentratiedrempel waarboven cellen geëxciteerd worden en de effectieve diffusieconstante. Dit theoretische raamwerk zorgt voor een intuïtief begrip en maakt analyse van experimentele resultaten mogelijk in deze vier termen. De theoretisch voorspelde golfvorm komt overeen met die van gemeten kalium concentraties bij ratten in de literatuur.

Hoofdstuk 5 behandelt de neurondynamica tijdens anoxische depolarisatie. Deze depolarisaties spelen een rol bij beroertes, migraine en epilepsie. Een van de effecten 
van energiedepletie is experimenteel gesimuleerd door de $\mathrm{Na} / \mathrm{K}$-pompen te blokkeren met ouabain. Hierbij is de membraanspanning van piramidale cellen gemeten. Tijdens de resulterende depolarisaties zijn vijf verschillende types dynamisch gedrag geobserveerd. Een bifurcatieanalyse van een Hodgkin-Huxley cel liet zien dat deze verschillende responsen allemaal vertoond kunnen worden door een en dezelfde cel met normaal functionerende ion kanalen. De specifieke respons wordt bepaald door het verloop van de intra- en extracellulair concentraties natrium en kalium.

In hoofdstuk 6 worden differentiaalvergelijkingen voor een neuraal massa model afgeleid. In tegenstelling tot bestaande modellen, zijn deze vergelijkingen geheel afgeleid van de eigenschappen van de enkele cellen en hun connecties. Een "sigmoidale functie" is afgeleid van de vuurfrequentie-curve van de enkele cel en de variantie van de synaptische input. Daarbij zijn de varianties van de vuurfrequenties en de synaptische conductanties bepaald. Het model reproduceert het dynamische gedrag van twee populaties Hodgkin-Huxley cellen die synaptisch gekoppeld zijn zeer accuraat. Dit maakt het mogelijk om de macroscopische activiteit te onderzoeken die ontstaan door kanaalziekten, kanaalblokkers of pathologische ionconcentraties.

Verscheidene vragen moeten nog beantwoord worden om medicatie te kunnen onderzoeken die ischemische schade voorkomt en diagnose van postanoxische patiënten te kunnen verbeteren. Een aanpak om deze vragen te beantwoorden met behulp van experimenten en mathematische modellen wordt beschreven. 


\section{Dankwoord (Acknowledgements)}

Met het schrijven van deze thesis sluit ik niet alleen vier jaar onderzoek af, maar is ook een geweldige periode van tien jaar in Enschede en de Universiteit Twente (UT) afgelopen voor mij. Ik ben dankbaar voor de mensen die ik hier heb mogen ontmoeten, om mee te leren, te werken, te praten, te kletsen, drinken en dansen, heel veel lol mee te hebben en met wie ik lief en leed heb gedeeld.

Allereerst bedank ik Linda en Chris. Linda, de afgelopen maanden waarin ik mijn thesis heb geschreven, zijn zwaar geweest. Jij hebt grotendeels ons leven gerund en geweldig voor mij en Chris gezorgd. Ik schijn lang geleden gezegd te hebben dat ik het onzin vond dat wetenschappers altijd hun partner bedanken bij dit soort gelegenheden. Nu weet ik echter waarom dat gedaan wordt. Liefste, je bent altijd mijn steun en toeverlaat geweest en ik hoop dat in de komende tijd ook weer voor jou te kunnen zijn. Chris, ondanks dat je dat nog niet weet heb je ook een beetje geholpen. Jouw oneindige, stralende vrolijkheid is aanstekelijk. 's Avonds zorgde je er voor dat ik alle gedachten over mijn thesis even van me af kon zetten. Iedere morgen zorg je er voor dat ik net als jij blij ben dat er weer een nieuwe dag is om te beleven. Je bent het allerliefste knulletje dat ik ken en papa's grote kerel. Ik heb geen moment gedacht dat het zo geweldig zou zijn om jou in mijn leven te hebben. Het zal nog even duren voordat je dit kunt lezen, en nog veel langer voordat je dit ook écht begrijpt.

Dan wil ik Bennie en Michel bedanken, mijn promotors, begeleiders en mentors. Bennie, jij hebt mij onder je hoede genomen. Het eerste jaar is voor mij een grote zoektocht geweest in een nieuw vakgebied. Jij hebt mij alle ruimte gegeven om mijn eigen weg te bepalen voor mijn onderzoek. Verder wist je altijd feilloos er de vinger op te leggen hoe we een nieuw geschreven artikel beter uit de verf konden laten komen, zelfs (of misschien wel juist) bij onderwerpen die wat verder van je af lagen. Maar vooral ben ik je dankbaar voor de motivatie, zorg en vaderlijke adviezen die je mij gegeven hebt. Michel, toen ik ongeveer een jaar bezig was, was het duidelijk dat mijn interesse om het brein te modelleren bijzonder goed aansloot bij jouw kennis en onderzoek. Ik kan me eigenlijk niet meer precies herinneren hoe ik nu een vast onderdeel van de zwaan-kleef-aan Clinische Neurophysiologie groep ben geworden. Dat is, in jouw woorden, heel organisch gegaan. Jouw unieke achtergrond als medicus en natuurkundige zorgde dat wij bijzonder goed konden praten en sparren. Ik heb 
erg genoten van onze geëngageerde gesprekken over neuro(patho)fysiologie, en over de wetenschap in het algemeen. Met jullie beiden was het altijd prettig samenwerken aan de geschreven artikelen.

Dan mijn collega's van de NIM en de CNPH groep en het ECTM. Het was bijzonder fijn om met jullie, een mix van mensen met verschillende achtergronden en persoonlijkheden, samen te werken, te discussiëren, te lunchen, te lachen, koffie te drinken, dingen te solderen, borrels en uitjes te houden, frustraties te delen, langs de viskraam te gaan, Sinterklaas te vieren, en goedmoedig elkaars discipline te dissen. $\mathrm{Na}$ zes jaar natuurkunde was het een aangename verandering om ook vrouwelijke collega's te hebben (waarbij Shaun dan mooi weer wat tegenwicht gaf in de CNPH groep). Ik zou over ieder van jullie graag een stuk tekst schrijven met waarom jullie zo geweldig zijn, maar dat zou een enorme klus zijn, waar ik nu de tijd niet voor wil nemen. Expliciet noem ik nog de verscheidene secretaresses, voor alles wat jullie voor mij geregeld hebben, Wim voor alles wat hij altijd voor ons geklust heeft en de ICTS voor hun snelle service. De studenten die ik heb mogen begeleiden als dagelijks of technisch begeleider tijdens Bachelor en Master projecten wil ik ook bedanken. Het was prettig samenwerken en jullie kwamen met interessante problemen en inzichten, waardoor ik zelf ook veel geleerd heb van jullie opdrachten. Evenzo ook de studenten die ik practicum en college heb gegeven. Jullie waren bijzonder geïnteresseerd, gemotiveerd en ook met jullie heb ik lol gehad.

I would like to thank the people I have collaborated with internationally. Professor Netoff for suggesting measurements of the anoxic depolarizations in his lab. Tyler for doing the measurements and discussing the results with me, as well as for the good times we had in Paris, and together with Abbey, teaching me some additions to my American English vocabulary. Professor Graf for hosting me in his institute and giving me the opportunity to do experiments in his lab, and for the fruitful discussions we had on spreading depression. I have learnt a lot during the experiments in the MPI and it is unfortunate that I have not been able to include this work in my thesis anymore. I would also like to thank the other people at the Max Planck Institute for neurological research in Cologne, especially Delphine and Heiko, for our discussions on SD, working together on the in vivo measurements, for which I also would like to thank Hiroyuki, the fun at the conferences and my days at the MPI, and for scaring the living daylights out of me when I was working late in the office.

Furthermore, I would like to thank the friendly, enthusiastic and sociable people I met at the conferences, especially the communities from the Cerebral Blood Flow \& Metabolism and the Computational Neuroscience conferences. The conferences have been useful and motivating, as well as a lot of fun: I never expected neuroscientists to throw such great parties.

Als laatste bedank ik dan nog kort de rest van de mensen die van mijn tien jaar op de UT zo'n mooie tijd hebben gemaakt: mijn oud-studiegenoten en doegroepbroert- 
jes, de leden en docenten van Arabesque, 4 happy feet, de balletgroep en Arashi, en mijn huisgenoten van het Pinohuis en Campuslaan 71.

Ik ga jullie missen. 


\section{Publications and Contributions}

\section{Journal publications}

- Bas-Jan Zandt, Sid Visser, Michel J.A.M. van Putten, Bennie ten Haken (2014) A neural mass model based on single cell dynamics to model pathologies. (submitted)

- Michel J.A.M. van Putten, Bas-Jan Zandt (2013) Neural mass modeling for predicting seizures. Clinical Neurophysiology, doi:10.1016/j.clinph.2013.11.013

- Bas-Jan Zandt, Tyler Stigen, Bennie ten Haken, Theoden Netoff, Michel JAM van Putten (2013) Single neuron dynamics during experimentally induced anoxic depolarization. Journal of Neurophysiology, 110:14691475

- Bas-Jan Zandt, Bennie ten Haken, Michel J.A.M. van Putten (2013) Diffusing substances during spreading depolarization: analytical expressions for propagation speed, triggering and concentration time-courses. The Journal of Neuroscience, 33(14):59155923

- Bas-Jan Zandt, Bennie ten Haken, Gert van Dijk, Michel JAM van Putten (2011) Neural Dynamics during Anoxia and the Wave of Death. PLOS ONE, 6(7):e22127. doi:10.1371/journal.pone.0022127

\section{Oral presentations}

- Bas-Jan Zandt, Bennie ten Haken, Michel J.A.M. van Putten, Modelling ion concentrations and neural dynamics during ischemia. (invited) Donders Discussions, Nijmegen 25-26 oktober 2012

- Bas-Jan Zandt, Bennie ten Haken, Michel J.A.M. van Putten, Expressions for initiation and propagation of spreading depolarization. COSBID meeting Londen, 21 september 2012

- Bas-Jan Zandt, Bennie ten Haken, Michel J.A.M. van Putten, Modelling failing ionic homeostasis after ischemic insults. Gordon research seminar, Waterville Maine, 11-17 August 2012 
- Bas-Jan Zandt, Bennie ten Haken, Michel J.A.M. van Putten, Modelling neuronal dynamics during brain ischemia. (invited) Workshop on Computational Approaches to etiology of Alzheimer's disease, Stockholm, Sweden, July 28, 2011

\section{Poster presentations}

- Bas-Jan Zandt, Sid Visser, Michel J.A.M. van Putten, Bennie ten Haken Modeling pathological brain rhythms: constructing a neural mass model from single cell dynamics. Computational NeuroScience 2013, Paris, July 13-18 2013

- Bas-Jan Zandt, Delphine Feuerstein, Heiko Backes, Hiroyuki Ima, Bennie ten Haken, Michel J.A.M. van Putten, Rudolf Graf, The role of diffusing potassium in spreading depolarization. Brain 2013, Shanghai, May 20-23 2013

- Bas-Jan Zandt, Bennie ten Haken and Michel J.A.M. van Putten, Modeling neuronal dynamics during brain ischemia. Computational NeuroScience 2011, Stockholm, July 23-28 2011

- Bas-Jan Zandt, Marleen C. Cloostermans, Sid Visser, Bennie ten Haken and Michel J.A.M. van Putten, Including the effects of hypoxia in simulations of neural networks. Brain 2011, Barcelona, May 25-28 2011 


\section{About the author}

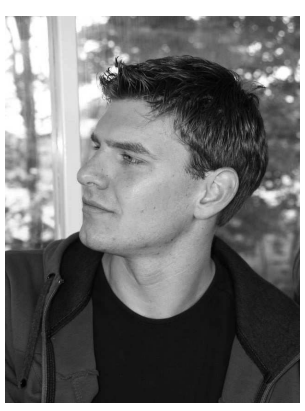

I was born 24 January 1985, in Kampen, the Netherlands and now live in Enschede, together with my wife Linda and newborn son Chris. In my free time, I am a fanatic amateur dancer, enjoy doing martial arts every now and then, or playing board games with my friends and family.

Loving the natural sciences and mathematics, I started my Bachelor in Aplied Physics in 2003 at the University of Twente, Enschede. For my Master in Applied Physics, I selected the optics and biophysics track, mainly focusing on laser physics and non-linear optics, liking the combination of experimental work and mathematics. During my Master, I performed a four month internship in the John Adams Institute for accelerater science in Oxford and to conclude my Master, I worked for a year in the Laser Physics and Non-linear Optics group.

After graduating I decided to switch to a different field, to leave the dark rooms with limitedly available equipment, but also to do research more closely related to clinical applications. As a $\mathrm{PhD}$-student in the VirtualPatient-Brain project, I was asked to investigate effects of ischemic insults measurable with EEG, MEG and/or MRI. I was given the freedom to define my own project and line of research, resulting in this thesis.

In my future career, I want to continue modeling the dynamics of neural activity and metabolism in diseases of the brain. In the last few years, more and more studies have successfully applied dynamical system theory to the brain. The dynamics of brain energetics and metabolism are found to play an important role in several neurological disorders, e.g. Alzheimers, epilepsy and stroke. The large majority of modeling studies, however, investigates either the healthy brain or epilepsy. I believe extending the dynamical system approach to other pathologies, such as ischemia/stroke, will eventually yield new treatments and diagnostics for a large group of patients. 
